\title{
MONOTONE REDUCIBILITY OVER THE CANTOR SPACE
}

\author{
RANDALL DOUGHERTY
}

\begin{abstract}
Define the partial ordering $\leq$ on the Cantor space ${ }^{\omega_{2}}$ by $x \leq y$ iff $\forall n x(n) \leq y(n)$ (this corresponds to the subset relation on the power set of $\omega$ ). A set $A \subseteq \omega_{2}$ is monotone reducible to a set $B \subseteq \omega_{2}$ iff there is a monotone (i.e., $x \leq y \Rightarrow f(x) \leq f(y)$ ) continuous function $f: \omega_{2} \rightarrow{ }^{\omega_{2}}$ such that $x \in A$ iff $f(x) \in B$. In this paper, we study the relation of monotone reducibility, with emphasis on two topics: (1) the similarities and differences between monotone reducibility on monotone sets (i.e., sets closed upward under $\leq$ ) and Wadge reducibility on arbitrary sets; and (2) the distinction (or lack thereof) between 'monotone' and 'positive,' where 'positive' means roughly 'a priori monotone' but is only defined in certain specific cases. (For example, a $\boldsymbol{\Sigma}_{2}^{0}$-positive set is a countable union of countable intersections of monotone clopen sets.) Among the main results are the following: Each of the six lowest Wadge degrees contains one or two monotone degrees (of monotone sets), while each of the remaining Wadge degrees contains uncountably many monotone degrees (including uncountable antichains and descending chains); and, although 'monotone' and 'positive' coincide in a number of cases, there are classes of monotone sets which do not match any notion of 'positive.'
\end{abstract}

\section{INTRODUCTION}

1. Definitions and conventions. Throughout this paper we will be considering the Cantor space ${ }^{\omega} 2$ with the partial ordering $\leq$ defined as follows: $x \leq y$ iff $x(n) \leq y(n)$ for all $n \in \omega$. (This corresponds to the subset relation under the canonical isomorphism between ${ }^{\omega} 2$ and the power set of $\omega$. We may also write $s \leq t$ where $s$ and $t$ are finite sequences from $\{0,1\}$ of the same length; the definition is analogous.) A subset of ${ }^{\omega_{2}}$ is called monotone iff it is closed upward under $\leq$; it is called hereditary iff it is closed downward under $\leq$.

The concept of Wadge reducibility is extremely useful in the study of subsets of the Cantor space and related spaces. (Recall that $X \subseteq{ }^{\omega}{ }_{2}$ is Wadge reducible to $Y \subseteq{ }^{\omega} 2$, written $X \leq_{\mathrm{w}} Y$, iff there is a continuous function $f:{ }^{\omega_{2}} \rightarrow{ }^{\omega_{2}}$ such that $X$ is the preimage of $Y$ under $f$.) However, Wadge reducibility is not quite as useful in the study of monotone subsets of ${ }^{\omega} 2$, because it does not preserve the order structure. A more useful notion in this context is the following: $X \leq_{\mathrm{m}} Y(X$ is monotone reducible to $Y$ ) iff there is a monotone continuous $f:{ }^{\omega} 2 \rightarrow{ }^{\omega} 2$ such that $X$ is the preimage of $Y$ under $f$. (A function $f:{ }^{\omega_{2}} \rightarrow{ }^{\omega_{2}}$ is monotone iff $f(x) \leq f(y)$ for all $x \leq y$ in ${ }^{\omega} 2$.)

Received by the editors March 12, 1987.

1980 Mathematics Subject Classification (1985 Revision). Primary 03E15; Secondary 04A15, $54 \mathrm{~F} 05$.

Key words and phrases. Cantor space, monotone sets, positive sets, Wadge reducibility, difference hierarchy, Borel hierarchy. 
This paper is devoted to a study of monotone reducibility on the monotone subsets of ${ }^{\omega_{2}}$. We will consider a number of properties of Wadge reducibility and determine whether their analogues hold in the monotone case. Along the way we will answer several questions of Cenzer [1]. Also, we consider a family of structural questions about monotone sets and translate it into a collection of questions about monotone reducibility, of which several are answered.

We now give a few conventions and notations. Unless stated otherwise, we will be working in the theory $\mathrm{ZF}+\mathrm{AC}_{\aleph_{0}}(\mathbf{R})$; that is, we will use standard set theory, but the only axiom of choice we will assume is choice from countably many sets of reals. The letters $A, B$, and $C$ will be reserved for monotone sets, while $Z$ will be used only for hereditary sets. (We will use $X$ and $Y$ for arbitrary sets of points, when needed.) Let Mon be the collection of monotone subsets of ${ }^{\omega_{2}}$. For any $X \subseteq{ }^{\omega} 2$, let $X^{\leq}$ denote the upward closure of $X$ (i.e., $\left\{y \in{ }^{\omega} 2:(\exists x \in X) x \leq y\right\}$ ), and let $X_{\leq}$denote the downward closure of $X ; \bar{X}$ will denote the topological closure of $X$, as usual. Given a set $S$ and a function $f$, let $f$ " $S$ and $f^{-1}$ " $S$ denote the image and preimage of $S$ under $f$, respectively. When working with sequences of numbers, we will use juxtaposition (of numbers or sequences) to denote concatenation; for example, $110 x$ is short for $\langle 1,1,0\rangle^{n} x$. Also, if $X \subseteq \omega_{2}$ and $i \in 2$, we write $i X$ for $\{i x: x \in X\}$; so $110^{\omega} 2$ is the set of members of ${ }^{\omega} 2$ starting with 110 . Borrowing the notation for repeating decimals, we will write $\overline{0}$ for the infinite sequence $\langle 0,0,0, \ldots\rangle, \overline{110}$ for $\langle 1,1,0,1,1,0, \ldots\rangle$, and so on. Let $\ell(s)$ denote the length of the finite sequence $s$.

For sets $X, Y \subseteq{ }^{\omega} 2$, define $X \equiv_{\mathrm{w}} Y$ to mean $X \leq_{\mathrm{w}} Y$ and $Y \leq_{\mathrm{w}} X$, and define $X<_{\mathrm{w}} Y$ to mean $X \leq_{\mathrm{w}} Y$ but $Y \mathbb{Z}_{\mathrm{w}} X$. The Wadge class of $X$ is the collection of all $Y \leq_{\mathrm{w}} X$, while the Wadge degree of $X$ is the collection of all $Y \equiv_{\mathrm{w}} X$. Analogous definitions are used for monotone reducibility. Unless otherwise noted, a monotone class or degree will always mean the class or degree of a monotone set under $\leq_{m}$. Since $\leq_{m}$ implies $\leq_{w}$, each monotone degree is contained within a Wadge degree.

2. Boolean operations. The notions of Wadge reducibility and monotone reducibility are closely related to the ranges of infinitary Boolean operations.

DEFINITION. An operation $H$ taking $\omega$-sequences of subsets of a given set $X$ to subsets of $X$ is Boolean iff there is a set $T(H) \subseteq \omega_{2}$ (the truth table of $H$ ) such that, for any sequence $\left\langle S_{n}: n \in \omega\right\rangle$ of subsets of $X$ and any $x \in X, x \in H\left(\left\langle S_{n}: n \in \omega\right\rangle\right)$ iff the characteristic function of $\left\{n \in \omega: x \in S_{n}\right\}$ is in $T(H)$.

So, of course, the countable union operation (which we denote by $\sigma$ to distinguish it from binary and unary unions) is Boolean, with truth table $\omega_{2}-\{\overline{0}\}$, as is the countable intersection operation (denoted by $\delta$ ), with truth table $\{\overline{1}\}$. Given a collection $\Gamma$ of subsets of $X$ and an operation $H$ as above, let $\Gamma_{H}$ be the collection of sets obtained by applying $H$ to all sequences from $\Gamma$; that is, $\Gamma_{H}=H$ "w $\Gamma$. Thus, if $\mathcal{G}$ and $\mathcal{F}$ are the collections of open sets and closed sets, respectively, then $\mathcal{G}_{\delta}$ and $F_{\sigma}$ will have their usual meanings. It makes sense to apply this notation to finitary Boolean operations as well. In this context, we use $\rho$ to denote the binary difference operation as distinguished from the unary complementation operation -; this means that $\mathcal{F}_{-}=\mathcal{G}$, while $\mathcal{F}_{\rho}$ is the collection of differences of closed sets. Also, we use $\mathrm{Cp}$ to denote the collection of clopen sets, since subscripting $\Delta_{1}^{0}$ is messy. 
In order to make notations such as $\mathcal{F}_{\sigma \delta}$ have their usual meanings, namely $\mathcal{F}_{\sigma \delta}=\left(\mathcal{F}_{\sigma}\right)_{\delta}$, we let $H_{0} H_{1}$ denote the operation obtained by applying $H_{0}$ and then $H_{1}$. Strictly speaking, the domain of this operation should consist of sequences of sequences of sets; however, if we fix a pairing function from $\omega \times \omega$ to $\omega$, we can rearrange such double sequences into ordinary sequences and vice versa, so we can consider $H_{0} H_{1}$ to be a mapping from $\omega$-sequences of sets to sets (which will be Boolean if $H_{0}$ and $H_{1}$ are). The same sort of rearrangement allows us to treat Suslin's operation $\mathcal{A}$ as an $\omega$-ary Boolean operation.

It is easy to see that a Boolean operation $H$ is monotone (i.e., $S_{n} \subseteq S_{n}^{\prime}$ for all $n$ implies $\left.H\left(\left\langle S_{n}: n \in \omega\right\rangle\right) \subseteq H\left(\left\langle S_{n}^{\prime}: n \in \omega\right\rangle\right)\right)$ iff its truth table is monotone.

The following proposition gives the main connection between Boolean operations and Wadge and monotone reducibility. We state the proposition for ${ }^{\omega} 2$, although the same proof works when $X$ is a subset of any (partially ordered) topological space. The first part is due to Miller and Radin [11, pp. 105-106].

PrOPOSITION 1.1. Let $H$ be an $\omega$-ary Boolean operation over $\omega_{2}$. Then, for any $X \subseteq{ }^{\omega}$,

$$
X \in \mathrm{Cp}_{H} \Leftrightarrow X \leq_{\mathrm{w}} T(H)
$$

and

$$
X \in(\mathrm{Cp} \cap \mathrm{Mon})_{H} \Leftrightarrow X \leq_{\mathrm{m}} T(H) .
$$

PROOF. If $X \in \mathrm{Cp}_{H}$, then $X=H\left(\left\langle S_{n}: n \in \omega\right\rangle\right)$ for some clopen sets $S_{n} \subseteq{ }^{\omega} 2$. Define $f:{ }^{\omega}{ }_{2} \rightarrow{ }^{\omega}{ }_{2}$ by: $f(x)(n)=1$ iff $x \in S_{n}$. Since the sets $S_{n}$ are clopen, $f$ is continuous; and the definition of $H$ from $T(H)$ gives $X=f^{-1}$ " $T(H)$, so $X \leq_{\mathrm{w}} T(H)$. Conversely, if $X \leq_{\mathrm{w}} T(H)$, say $X=f^{-1}$ " $T(H)$ where $f:{ }^{\omega} 2 \rightarrow{ }^{\omega} 2$ is continuous, then let $S_{n}=\left\{x \in{ }^{\omega_{2}}: f(x)(n)=1\right\}$; the continuity of $f$ implies that the sets $S_{n}$ are clopen, and the definition of $H$ from $T(H)$ gives $X=H\left(\left\langle S_{n}: n \in \omega\right\rangle\right)$.

The proof of the second part is identical; simply note that $f$ is monotone iff the sets $S_{n}$ are monotone.

So $\mathrm{Cp}_{H}$ is a Wadge class, namely that of $T(H)$, and $(\mathrm{Cp} \cap \mathrm{Mon})_{H}$ is the monotone class of $T(H)$. Note that this latter statement is true even if $T(H)$ is not monotone; however, as noted before, we will be using it only in the case that $T(H)$ is monotone (i.e., $H$ is monotone).

3. Monotone and positive sets. Many of the Wadge classes we will be looking at are of the form $\mathrm{Cp}_{H}$ for some monotone $\omega$-ary Boolean operation $H$ (in fact, as we will see later, they all are). A set in such a class can be monotone even if the clopen sets it is obtained from are not monotone; however, if the clopen sets $C_{n}$ are monotone, the set $H\left(\left\langle C_{n}: n \in \omega\right\rangle\right)$ will automatically be monotone. A set is called $H$-positive if it is in $(\mathrm{Cp} \cap \mathrm{Mon})_{H}$; thus, the $H$-positive sets are the "a priori monotone" sets in $\mathrm{Cp}_{H}$.

If a Wadge class $\Gamma$ is naturally defined as $\mathrm{Cp}_{H}$ for some monotone Boolean operation $H$, then we may use the term $\Gamma$-positive instead of $H$-positive. In particular, we use $\Sigma_{1}^{0}$-positive instead of $\sigma$-positive, $\Pi_{1}^{0}$-positive instead of $\delta$-positive, $\boldsymbol{\Sigma}_{2}^{0}$-positive instead of $\delta \sigma$-positive, and so on. This continues through all levels of the Borel hierarchy; in defining operations for the classes $\Sigma_{\alpha}^{0}$ and $\Pi_{\alpha}^{0}$, choices must be made to handle limit stages, but the resulting operations are easily seen to generate the same monotone classes (i.e., their truth tables are monotone-equivalent). The resulting definition is equivalent to: For $\alpha>1$, a set $A \subseteq{ }^{\omega} 2$ is $\Sigma_{\alpha}^{0}$-positive 
( $\boldsymbol{\Pi}_{\alpha}^{0}$-positive) iff it is a union (intersection) of countably many sets, each of which is $\Pi_{\beta}^{0}$-positive $\left(\Sigma_{\beta}^{0}\right.$-positive) for some $\beta<\alpha$.

Since Suslin's operation $A$ generates the analytic $\left(\Sigma_{1}^{1}\right)$ subsets of $\omega_{2}$ (this is a standard result; see section III.6), we define $\Sigma_{1}^{1}$-positive to mean $A$-positive.

Just knowing that a set in $\mathrm{Cp}_{H}$ is monotone does not tell us much about the structure of the set in $\left({ }^{\omega} 2, \leq\right)$; the set may be monotone "by coincidence." On the other hand, if we know that the set is $H$-positive, we know how it is built up from monotone sets, and this can give useful structural information. Therefore, an assertion of the form

$$
(\mathrm{Cp} \cap \mathrm{Mon})_{H}=\mathrm{Cp}_{H} \cap \mathrm{Mon}
$$

would be very useful if true. (The left-to-right inclusion of (1.1) just says that $H$ is monotone; the other inclusion says that every monotone set in $\mathrm{Cp}_{H}$ is $H$ positive.) By Proposition 1.1, (1.1) is equivalent to saying that $T(H)$ is a $\leq_{\mathrm{m}^{-}}$greatest monotone set in its Wadge class; therefore, one of the questions we will consider in part III for each of various Wadge classes is what its greatest monotone degree is (or even whether it has a greatest monotone degree).

We conclude this section by proving that (1.1) holds for $\sigma, \delta, \delta \sigma$, and $\sigma \delta$. This fact, as well as a number of the definitions given so far, appears in Cenzer [1].

Proposition 1.2. (a) For any $X \subseteq{ }^{\omega_{2}}, \bar{X}^{\leq}=\overline{X \leq}$.

(b) If $\Gamma$ is $\Sigma_{1}^{0}, \Pi_{1}^{0}, \Sigma_{2}^{0}$, or $\Pi_{2}^{0}$, then any monotone $\Gamma$ subset of ${ }^{\omega_{2}}$ is $\Gamma$-positive.

Proof. (a) Suppose $y \in \bar{X}^{\leq}$. Then there is $x \leq y$ such that $x \in \bar{X}$, which means that for each $n \in \omega$ there is $x^{\prime} \in X$ such that $x^{\prime}\lceil n=x\lceil n$. This implies $\left(y\lceil n) \overline{1} \geq x^{\prime}\right.$, so $(y\lceil n) \overline{1} \in X \leq$; since this is true for all $n, y \in \overline{X \leq}$.

Conversely, suppose $y \in \overline{X \leq}$. Then for each $n \in \omega$ there is $y^{\prime} \in X \leq$ such that $y^{\prime}\lceil n=y\lceil n$, so there is $x \in X$ such that $x\lceil n \leq y\lceil n$. Applying König's infinity lemma, we get $x \in \bar{X}$ such that $x \leq y$, so $y \in \bar{X}$.

(b) If $X \subseteq{ }^{\omega} 2$ is clopen, then $X$ has finite support (i.e., whether a point $x$ is in $X$ depends only on $x\lceil n$, for some fixed $n \in \omega)$; this follows from the König infinity lemma. But then it is easy to see that $X \leq$ has the same finite support, so $X \leq$ is clopen. Also, it follows from (a) that, if $X$ is closed, then $X \leq$ is closed.

Now, if $A \subseteq{ }^{\omega_{2}}$ is monotone and $\Sigma_{1}^{0}$, then $A$ is the union of countably many clopen sets $X_{n}$, so $A$ is the union of the monotone clopen sets $X_{n} \leq$ and is therefore $\Sigma_{1}^{0}$-positive. By a duality argument (the complement of a monotone $\boldsymbol{\Pi}_{1}^{0}$ set is a hereditary $\Sigma_{1}^{0}$ set; see section II.1), any monotone $\Pi_{1}^{0}$ set is $\Pi_{1}^{0}$-positive. If $A$ is monotone and $\Sigma_{2}^{0}$, then $A$ is the union of countably many closed sets $X_{n}$; the sets $X_{n} \leq$ are $\Pi_{1}^{0}$-positive and their union is $A$, so $A$ is $\Sigma_{2}^{0}$-positive. Now duality gives the result for $\Pi_{2}^{0}$, so we are done.

\section{General CONSTRUCTIONS}

1. Isomorphisms and duality. The operation of complementation is a selfinverse automorphism of $\left(P\left(\omega_{2}\right), \leq_{\mathrm{w}}\right)$, since any function witnessing $X \leq_{\mathrm{w}} Y$ also witnesses $-X \leq_{\mathrm{w}}-Y$. This imposes a duality on the Wadge classes and degrees; for example, from the fact that there are exactly four Wadge degrees of open sets one can immediately conclude that there are exactly four Wadge degrees of closed sets. The complementation operation itself does not induce a duality on the monotone 
degrees (of monotone sets), since the complement of a monotone set is hereditary rather than monotone; however, a simple modification of complementation will induce such a duality.

Define the function $\mathbb{1}^{\omega_{2}} \rightarrow{ }^{\omega_{2}}$ by $\mathbb{1}(x)=\langle 1-x(0), 1-x(1), \ldots\rangle$. This is clearly a self-inverse automorphism of ${ }^{\omega_{2}}$ and an isomorphism between $\left({ }^{\omega} 2, \leq\right)$ and $\left({ }^{\omega} 2, \geq\right)$. Hence, the image of a monotone set under $\hat{1}$ is a hereditary set, and vice versa. Also, the function $\mathbb{v}$ witnesses $X \equiv_{\mathrm{w}} \hat{\Downarrow}$ " $X$ for any $X \subseteq{ }^{\omega_{2}}$, and the operation $X \mapsto \hat{\Downarrow}$ " $X$ commutes with Boolean operations (since it is the same as $X \mapsto \hat{\Downarrow}^{-1}$ " $X$ ); in particular, it commutes with complementation. Therefore, the operation $X \mapsto-1$ " $X$ induces the same duality on Wadge reducibility that complementation does; if $f$ witnesses $X \leq_{\mathrm{w}} Y$, then $\uparrow \circ f \circ \hat{1}$ witnesses $(-\hat{\mathbb{1}}$ “ $X) \leq_{\mathrm{w}}$ $(-\hat{\Downarrow} " Y)$. But $-\hat{\Downarrow}$ " $A$ is monotone if $A$ is, and $\hat{\Downarrow} \circ f \circ \hat{\Downarrow}$ is monotone if $f$ is, so this operation also induces a duality on monotone reducibility.

We have already run into one application of this duality, in Proposition 1.2. The dual of the collection $\mathcal{F}$ of closed sets is $\mathcal{F}_{-\uparrow}=\mathcal{G}$ (here and subsequently we abuse notation slightly by writing $\$$ instead of 1 "), the dual of $\sigma$ is $\delta$, and $\mathrm{Cp}$ and Mon are self-dual, so the dual of (1.1) for $\sigma$ is (1.1) for $\delta$; the case of $\Sigma_{2}^{0}$ and $\Pi_{2}^{0}$ is similar. We will be applying duality many times in the rest of the paper, sometimes without explicit mention. The operations defined in $\S \S 3$ and 4 preserve duality; this makes it even more useful.

One more isomorphism which will be useful later is the map $f:{ }^{\omega} 2 \times{ }^{\omega_{2}} \rightarrow{ }^{\omega_{2}}$ defined by $f(x, y)(2 n)=x(n)$ and $f(x, y)(2 n+1)=y(n)$. In fact, if we define $\leq$ on ${ }^{\omega_{2}} \times{ }^{\omega_{2}}$ in the obvious way (i.e., $(x, y) \leq\left(x^{\prime}, y^{\prime}\right)$ iff $x \leq x^{\prime}$ and $\left.y \leq y^{\prime}\right)$, then $f$ is an isomorphism between $\left({ }^{\omega} 2 \times{ }^{\omega} 2, \leq\right)$ and $\left({ }^{\omega} 2, \leq\right)$, so any definitions and results for ${ }^{\omega} 2$ can be transferred immediately to ${ }^{\omega} 2 \times{ }^{\omega} 2$.

2. Frontiers and a diagram representation. We will be constructing many specific monotone sets in part III, and in a number of cases it will be much easier to define these sets pictorially than textually. We therefore give a method for defining monotone subsets of ${ }^{\omega} 2$ pictorially.

The Cantor space is usually visualized as the "middle thirds" subset of $[0,1]$, which is often depicted using a sequence of approximations (Figure 2.1(a)). Each segment in one of these approximations can be identified with a basic clopen subset of ${ }^{\omega} 2$, as shown. This picture gives a good idea of the topological properties of ${ }^{\omega_{2}} 2$, but does not reflect the partial ordering we are studying.

To depict the ordering, we move to a planar representation of ${ }^{\omega} 2$ (Figure 2.1(b)). Instead of dividing segments into two pieces at each step to show the next coordinate of a point in ${ }^{\omega_{2}}$, we divide squares into four pieces at each step to represent the next two coordinates of the point. The incomparable sets $01^{\omega} 2$ and $10^{\omega} 2$ are at the same level in the picture, and the set $11^{\omega} 2$ lies above them in both the ordering and the depiction. The representation is not perfect, of course (for example, the point representing $11 \overline{0}$ lies above that representing $00 \overline{1})$, but it works well enough to get an intuitive idea of the ordering.

Now we want to depict a monotone set $A \subseteq{ }^{\omega_{2}}$. We will do this by placing two symbols within the outer square representing ${ }^{\omega} 2$ (the inner squares will usually not be drawn): a filled-in circle to represent a frontier point of $A$ which lies in $A$ (i.e., a member of $A \cap \overline{-A}$ ) and an unfilled circle to represent a frontier point of $A$ which is not in $A$ (a member of $\bar{A}-A$ ). Two such circles will be connected 
(a) Unordered:

$\omega_{2}$
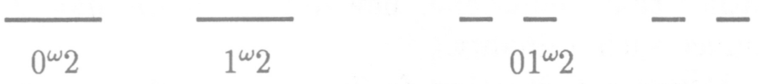

(b) Ordered:
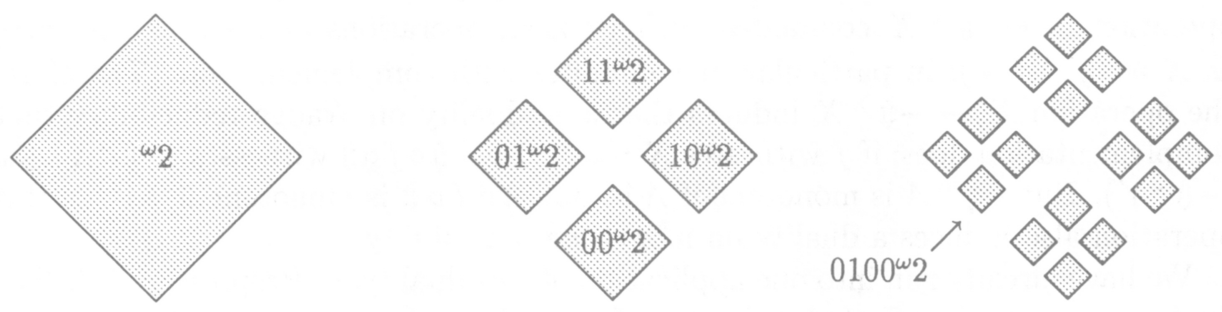

FiguRE 2.1. Diagrams for the Cantor space

by a nearly-vertical (or at least nonhorizontal) segment to indicate that one of the corresponding points lies above the other in the partial ordering; if two such points are not required by such segments to be comparable, they are assumed to be incomparable. Hence, the diagrams in Figure 2.2 depict two monotone subsets of $\omega_{2}$, each having two frontier points; in (a) the two frontier points are incomparable, while in (b) the one in the set lies above the one not in the set.

It is hard to depict a set with uncountably many frontier points using this representation, but we will rarely need such sets. Two more serious objections to this representation are: One cannot tell just which point in ${ }^{\omega_{2}} 2$ is represented by a given circle, and the diagram does not indicate which nonfrontier points are in the set. This would seem to indicate that one cannot define a specific monotone subset of $\omega_{2}$ using these diagrams; however, the following two propositions will show that, if we only care about defining sets up to monotone equivalence, the diagrams will suffice. Let $\operatorname{Fr}(X)$ denote the frontier of the set $X$.

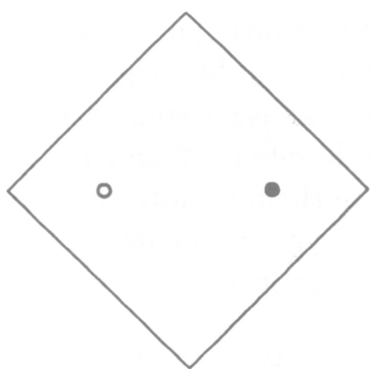

(a)

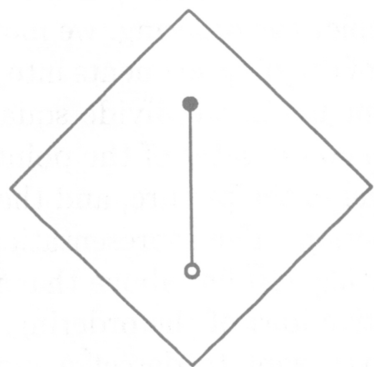

(b)

Figure 2.2. Two subsets of the Cantor space 
Proposition 2.1. Suppose $S \subseteq \omega_{2}$ is closed and $\operatorname{Mon}_{\rho}$ (the difference of two monotone sets), and $T$ is a relatively monotone subset of $S$ (i.e., $T \leq \cap S=T$ ). Assume that $S$ contains no eventually constant sequences. Then there is a monotone $A \subseteq{ }^{\omega} 2$ such that $\operatorname{Fr}(A)=S$ and $A \cap S=T$.

Proposition 2.2. Suppose $A, B \subseteq{ }^{\omega} 2$ are monotone, $\operatorname{Fr}(A)$ is nonempty, and there is a continuous function $f: \operatorname{Fr}(A) \rightarrow \operatorname{Fr}(B)$ which is relatively monotone (i.e., $f(x) \leq f(y)$ for any $x, y \in \operatorname{Fr}(A)$ such that $x \leq y)$ and satisfies $x \in A \Leftrightarrow f(x) \in B$ for each $x \in \operatorname{Fr}(A)$. Then there is $g:{ }^{\omega} 2 \rightarrow{ }^{\omega} 2$ extending $f$ and witnessing $A \leq_{\mathrm{m}} B$.

Proposition 2.1 states that, for almost any reasonable specification of the frontier of a monotone set, there actually is a monotone set meeting that specification. (Clearly the frontier of a monotone set $A$ is closed and $\operatorname{Mon}_{\rho}$, since $\operatorname{Fr}(A)=\bar{A} \cap \overline{-A}$; Proposition 1.2(a) implies that $\bar{A}$ is monotone, and its dual implies that $\overline{-A}$ is hereditary. The extra assumption in 2.1 is annoying and stronger than absolutely necessary, but it cannot be deleted entirely; putting $S=T={ }^{\omega_{2}}$ would not work. However, it is always easy to get around this requirement; for example, one can replace $S$ and $T$ by their images under the function which maps $x \in{ }^{\omega} 2$ to $\langle 0,1, x(0), 0,1, x(1), \ldots\rangle$.) Proposition 2.2 implies that this monotone set is unique up to monotone equivalence; in fact, the monotone degree of the set depends only on the internal structure of $S$ and $T$, not on how they are embedded in ${ }^{\omega_{2}} 2$. Hence, almost any diagram as in this section defines a unique monotone degree. (Sometimes an explicit specification of $S$ and $T$ will accompany a diagram when it is not obvious that the diagram can be embedded in ${ }^{\omega} 2$.) Furthermore, when one is trying to decide whether one monotone set is monotone reducible to another, the diagrams give all of the required information.

We now prove the two propositions. Note the useful fact that, if $x$ is a point in the frontier of a monotone set $A$, then $(x \mid n) \overline{1} \in A$ and $(x\lceil n) \overline{0} \notin A$ for each $n \in \omega$. (Since $x \in \operatorname{Fr}(A)$, some point $y$ of $\left(x\lceil n)^{\omega} 2\right.$ is in $A$, and $(x \mid n) \overline{1} \geq y$; similarly for $(x\lceil n) \overline{0}$.

PROOF OF 2.1. First note that a set $X \subseteq{ }^{\omega} 2$ is $\operatorname{Mon}_{\rho}$ iff, whenever $x, z \in X$ and $x<y<z, y$ is also in $S$. If $X=B-C, x, z \in X$, and $x<y<z$, then $y \in B$ since $z \in B$ and $y \notin C$ since $x \notin C$, so $y \in X$. Conversely, if $X$ has the latter property, then it is easy to see that $X=X \leq \cap X_{\leq}$.

The sets $B=-\left(S_{\leq}\right)$and $Z=-(S \leq)$ are both open. Since $S$ is $\mathrm{Mon}_{\rho}, S=$ $S \leq \cap S_{\leq}$, so $B \cup Z=-S$. By the polarized first separation property for open sets (see §III.5), there are $B^{\prime} \subseteq B$ and $Z^{\prime} \subseteq Z$ (with $B^{\prime}$ monotone and $Z^{\prime}$ hereditary, by our notational convention) such that $B^{\prime} \cup Z^{\prime}=-S$ and $B^{\prime} \cap Z^{\prime}=\varnothing$. Let $A=B^{\prime} \cup T$. Since $T \leq \cap S=T$ and $T \leq-S \subseteq S \leq-S \subseteq B-Z \subseteq B^{\prime}, A$ is monotone. Since $B^{\prime} \cap S=\varnothing, A \cap S=T$. Since $B^{\prime} \subseteq A$ and $Z^{\prime} \subseteq-A$ are open, $\operatorname{Fr}(A) \subseteq-\left(B^{\prime} \cup Z^{\prime}\right)=S$. Now suppose $x \in S$ and $n \in \omega$. The point $(x \mid n) \overline{1}$ is not in $S$ by hypothesis and it is not in $Z^{\prime}$ since $\left(x\lceil n) \overline{1} \geq x \notin Z^{\prime}\right.$, so $\left(x\lceil n) \overline{1} \in B^{\prime} \subseteq A\right.$. Similarly, $\left(x\lceil n) \overline{0} \in Z^{\prime} \subseteq-A\right.$. Since $(x \mid n) \overline{1} \rightarrow x$ and $(x\lceil n) \overline{0} \rightarrow x$ as $n \rightarrow \infty$, $x \in \operatorname{Fr}(A)$; since $x$ was arbitrary, $S=\operatorname{Fr}(A)$, so we are done.

PROOF OF 2.2. Let $S=\operatorname{Fr}(A)$. For each $x \in \omega_{2}-S$, there is an $n \in \omega$ such that $x \uparrow(n+1)$ decides membership in $A$ (i.e., either $\left(x\lceil(n+1))^{\omega_{2}} \subseteq A\right.$ or $\left.(x \uparrow(n+1))^{\omega} 2 \subseteq-A\right)$; let $d(x)$ be the least such $n$. Since $S \neq \varnothing$ and $A$ is monotone, it is easy to see that $\left(x\lceil(d(x)+1))^{\omega} 2 \subseteq A\right.$ iff $x(d(x))=1$. Define the function $h$ by: 
for each $n \in \omega$ such that there is $x \in{ }^{\omega} 2-S$ with $d(x) \geq n$, let $h(n)$ be the least $k$ such that there is $x \in \omega_{2}-S$ with $d(x) \geq n$ and $(x \uparrow(k+1))^{\omega} 2 \cap S=\varnothing$. Clearly $h$ is a nondecreasing function on its domain.

Claim. If $h(n)$ is defined for all $n \in \omega$, then $h(n) \rightarrow \infty$ as $n \rightarrow \infty$.

ProOF. Suppose not, and fix $k \in \omega$ such that $k>h(n)$ for all $n$; then we can choose $x_{n} \in{ }^{\omega} 2$ for each $n$ such that $d(x) \geq n$ and $\left(x_{n}\lceil k)^{\omega} 2 \cap S=\varnothing\right.$. By replacing $\left\langle x_{n}: n \in \omega\right\rangle$ with a subsequence, we may assume that $x_{n}$ converges to some point $x \in{ }^{\omega} 2$ as $n \rightarrow \infty$. Since $d(x) \geq n,\left(x_{n}\lceil n)^{\omega} 2\right.$ meets both $A$ and $-A$; since $A$ is monotone, $\left(x_{n}\lceil n) \overline{1} \in A\right.$ and $\left(x_{n}\lceil n) \overline{0} \in-A\right.$. But $\left(x_{n}\lceil n) \overline{1} \rightarrow x\right.$ and $\left(x_{n}\lceil n) \overline{0} \rightarrow x\right.$ as $n \rightarrow \infty$, so $x \in S$. Since $x_{n} \rightarrow x$, there is $n$ such that $x\left\lceil k=x_{n}\lceil k\right.$, so $\left(x_{n}\lceil k)^{\omega} 2 \cap S \neq \varnothing\right.$, contradiction.

Now define $g:{ }^{\omega} 2 \rightarrow{ }^{\omega}{ }_{2}$ by

$$
g(x)= \begin{cases}f(x) & \text { if } x \in S, \\ ((\operatorname{lub}\{f(y): y \in S, y\lceil h(d(x)) \leq x\lceil h(d(x))\})\lceil h(d(x))) \overline{1} & \text { if } x \in A-S, \\ ((\operatorname{glb}\{f(y): y \in S, y\lceil h(d(x)) \geq x\lceil h(d(x))\})\lceil h(d(x))) \overline{0} & \text { if } x \in-A-S .\end{cases}
$$

Here lub and glb stand for the least upper bound and greatest lower bound, respectively, of a subset of ${ }^{\omega_{2}}$. It follows immediately from the definition of $h$ that $(x \nmid h(d(x)))^{\omega} 2 \cap S \neq \varnothing$; hence, the arguments to lub and glb above are nonempty.

First, we show that $g(x) \in B$ iff $x \in A$. This is true by hypothesis if $x \in S$. If $x \in A-S$, choose $y \in S$ such that $y\lceil h(d(x))=x\lceil h(d(x))$; then $f(y) \in \operatorname{Fr}(B)$, so $(f(y)\lceil h(d(x))) \overline{1} \in B$, and since $g(x) \geq(f(y)\lceil h(d(x))) \overline{1}, g(x) \in B$. The argument for $x \in-A-S$ is analogous.

We now show that $g$ is continuous. If $x \notin S$, then $g(x)$ depends only on $x\lceil h(d(x))$. But $d(y)=d(x)$ if $y \uparrow(d(x)+1)=x \uparrow(d(x)+1)$, so $g(y)=g(x)$ if $y\lceil k=x\lceil k$, where $k=\max (h(d(x)), d(x)+1)$; hence, $g$ is continuous at $x$. Now suppose $x \in S$. If a neighborhood of $x$ is contained in $S$, then $g$ is continuous at $x$ since it matches $f$ on this neighborhood; so suppose this is not the case. Fix $m \in \omega$; we will find $n \in \omega$ so large that $x^{\prime} \mid n=x\left\lceil n\right.$ implies $f\left(x^{\prime}\right) \mid m=f(x)\lceil m$. There is $k \in \omega$ so large that $f(y)\lceil m \leq f(x)\lceil m$ for any $y \in S$ such that $y\lceil k \leq x\lceil k$ and $f(y)\lceil m \geq f(x)\lceil m$ for any $y \in S$ such that $y\lceil k \geq x\lceil k$. (This follows from a compactness argument similar to that in Proposition 1.2(a).) Note that, if $x^{\prime} \nmid n=x \mid n$ but $x^{\prime} \notin S$, then $d\left(x^{\prime}\right) \geq n$. Now choose $n$ so large that $n \geq k, h(n) \geq k$, and $h(n) \geq m$. To see that this $n$ works, suppose $x^{\prime} \in{ }^{\omega} 2$ and $x^{\prime}|n=x| n$. If $x^{\prime} \in S$, then $f\left(x^{\prime}\right) \mid m=f(x)\lceil m$ since $n \geq k$. If $x^{\prime} \in A-S$, then $d\left(x^{\prime}\right) \geq n$, so $h\left(d\left(x^{\prime}\right)\right) \geq k$. This means that each $y$ considered in the definition of $g\left(x^{\prime}\right)$ satisfies $y\left\lceil k \leq x^{\prime}|k=x| k\right.$, so $f(y) \mid m \leq f(x)\lceil m$ for all such $y$. But there is such a $y$ for which $y\left\lceil h\left(d\left(x^{\prime}\right)\right)=x^{\prime} \nmid h\left(d\left(x^{\prime}\right)\right)\right.$, so $y\lceil k=x\lceil k$, so $f(y) \mid m=f(x)\left\lceil m\right.$. Therefore, $g\left(x^{\prime}\right)\lceil m=f(x)\lceil m=g(x)\lceil m$, as desired. The case $x^{\prime} \in-A-S$ is analogous.

It remains to show that $g$ is monotone. Suppose $x, x^{\prime} \in{ }^{\omega_{2}}$ and $x \leq x^{\prime}$. Note that $-A-S$ is hereditary since it is the complement of the monotone set $\bar{A}$, and similarly $A-S$ is monotone; this reduces the number of cases we must consider to six. If $x, x^{\prime} \in S$, then $g(x)=f(x) \leq f\left(x^{\prime}\right)=g\left(x^{\prime}\right)$ since $f$ is relatively monotone. If $x \in S$ and $x^{\prime} \in A-S$, then $x$ is one of the $y$ 's considered in the definition of $g\left(x^{\prime}\right)$, so $g\left(x^{\prime}\right) \geq f(x)=g(x)$; similarly if $x \in-A-S$ and $x^{\prime} \in S$. Next, suppose $x, x^{\prime} \in A-S$. Then $\left(x\lceil(d(x)+1))^{\omega} 2 \subseteq A\right.$, so, since $A$ is monotone, 
$\left(x^{\prime} \uparrow(d(x)+1)\right)^{\omega_{2}} \subseteq A$; therefore, $d\left(x^{\prime}\right) \leq d(x)$, and hence $h\left(d\left(x^{\prime}\right)\right) \leq h(d(x))$. But then each $y$ considered in the definition of $g(x)$ is also considered for $g\left(x^{\prime}\right)$, so $g\left(x^{\prime}\right)\left\lceil h\left(d\left(x^{\prime}\right)\right) \geq g(x)\left\lceil h\left(d\left(x^{\prime}\right)\right)\right.\right.$, so $g\left(x^{\prime}\right) \geq g(x)$. Finally, suppose $x \in-A-S$ and $x^{\prime} \in A-S$. Assume $d(x) \geq d\left(x^{\prime}\right)$; the case $d(x) \leq d\left(x^{\prime}\right)$ is similar. Choose $y \in S$ such that $y\lceil h(d(x))=x\lceil h(d(x))$. Then $y$ meets the conditions in the definitions of both $g(x)$ and $g\left(x^{\prime}\right)$, so $g(x) \leq f(y) \leq g\left(x^{\prime}\right)$, as desired.

3. Least upper bounds. For any sets $X, Y \subseteq \omega_{2}$, define $\operatorname{jn}(X, Y)$ to be $0 X \cup 1 Y$. It is easy to see that $\operatorname{jn}(X, Y)$ is a least upper bound of $X$ and $Y$ under Wadge reducibility, since $0^{\omega} 2$ and $1^{\omega} 2$ are clopen. (This immediately implies that $\mathrm{jn}\left(X^{\prime}, Y^{\prime}\right) \leq_{\mathrm{w}} \mathrm{jn}(X, Y)$ whenever $X^{\prime} \leq_{\mathrm{w}} X$ and $Y^{\prime} \leq_{\mathrm{w}} Y$.) Furthermore, $-\mathrm{jn}(X, Y)=\mathrm{jn}(-X,-Y)$. We will define a version of jn for monotone sets under monotone reducibility with analogous properties.

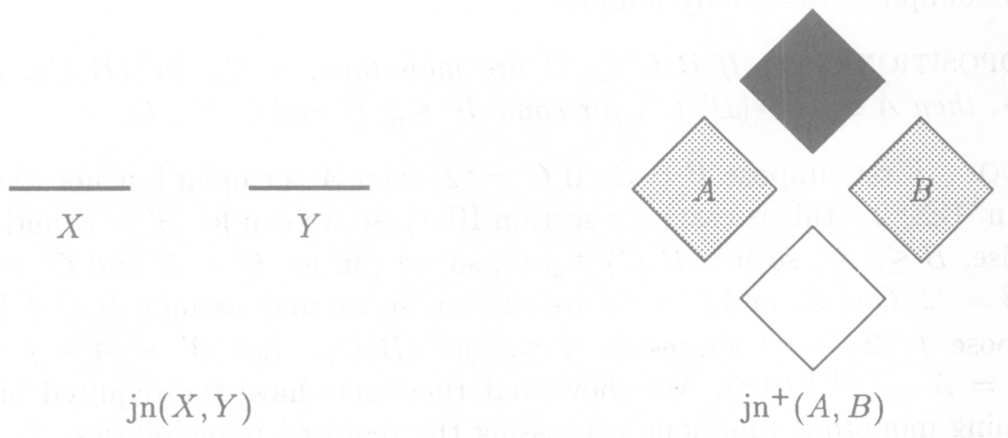

FIGURE 2.3. The operations jn and jn ${ }^{+}$

For monotone sets $A, B \subseteq \omega_{2}$, define $\mathrm{jn}^{+}(A, B)$ as follows: if $A=B=\varnothing$ or $A=B={ }^{\omega_{2}}$, let jn ${ }^{+}(A, B)=A$; otherwise, let jn ${ }^{+}(A, B)=01 A \cup 10 B \cup 11^{\omega} 2$. (See Figure 2.3. The first two cases are not as exceptional as they appear, as we will see in the next section.) Clearly $\mathrm{jn}^{+}(A, B)$ is monotone and $A$ and $B$ are monotone reducible to it. Now, suppose $C \geq_{\mathrm{m}} A$ and $C \geq_{\mathrm{m}} B$. If $A=B=\varnothing$ or $A=B={ }^{\omega} 2$, then clearly $C \geq_{\mathrm{m}} \mathrm{jn}^{+}(A, B)$. Otherwise, we must have $C \neq \varnothing$ and $C \neq{ }^{\omega_{2}}$, so $\overline{1} \in C$ and $\overline{0} \notin C$. Hence, if monotone functions $f$ and $g$ reduce $A$ and $B$, respectively, to $C$, then $\mathrm{jn}^{+}(A, B)$ is reduced to $C$ by the monotone function $h$ defined by $h(01 x)=f(x), h(10 x)=g(x), h(11 x)=\overline{1}$, and $h(00 x)=\overline{0}$. This proves that $\mathrm{jn}^{+}(A, B)$ is a least upper bound of $A$ and $B$ with respect to monotone sets and monotone reducibility.

A similar argument shows that $\mathrm{jn}^{+}(A, B)$ is a least upper bound of $A$ and $B$ with respect to $\leq_{\mathrm{w}}$; one merely chooses two suitable points instead of using the fixed points $\overline{1}$ and $\overline{0}$. Therefore, the collection of monotone sets in the monotone or Wadge class or degree of a given monotone set is closed under $\mathrm{jn}^{+}$(since the given set is an upper bound for any two sets in the collection). This means that a member of this collection is $\leq_{\mathrm{m}}$-greatest in the collection (i.e., exceeds or is equivalent to any other member) iff it is $\leq_{m}$-maximal in the collection (i.e., is not exceeded by any other member). Also, a monotone set is $\leq_{m}$-maximal in its Wadge class iff it is $\leq_{\mathrm{m}}$-maximal in its Wadge degree. 
If $A \subseteq A^{\prime}$ and $B \subseteq B^{\prime}$, then jn ${ }^{+}(A, B) \subseteq \mathrm{jn}^{+}\left(A^{\prime}, B^{\prime}\right)$. Also, if $A_{n} \subseteq A_{n+1}$ and $B_{n} \subseteq B_{n+1}$ for $n \in \omega$, then jn ${ }^{+}\left(\bigcup_{n \in \omega} A_{n}, \bigcup_{n \in \omega} B_{n}\right)=\bigcup_{n \in \omega} \mathrm{jn}^{+}\left(A_{n}, B_{n}\right)$, and if $A_{n} \supseteq A_{n+1}$ and $B_{n} \supseteq B_{n+1}$ for $n \in \omega$, then $\mathrm{jn}^{+}\left(\bigcap_{n \in \omega} A_{n}, \bigcap_{n \in \omega} B_{n}\right)=$ $\bigcap_{n \in \omega} \mathrm{jn}^{+}\left(A_{n}, B_{n}\right)$. (Another way of saying this is that the operation $\mathrm{jn}^{+}$is 'monotone' and 'continuous.') This is easy to prove; one merely has to be careful of the 'exceptional' cases, for which one uses such facts as: if $A_{n} \neq \omega^{\omega} 2$ for all $n$, then $\bigcup_{n} A_{n} \neq{ }^{\omega} 2$ (since $\left.\overline{0} \notin \bigcup_{n} A_{n}\right)$.

The set $\mathrm{jn}^{+}(B, C)$ is composed of four pieces in disjoint clopen parts of ${ }^{\omega} 2$, two of which look like $B$ and $C$ while the other two are trivial. Any set $A$ monotone reducible to this set will also be divided in this way, although some of the clopen parts might be empty. It therefore makes sense to suppose that $A$ is equivalent to a least upper bound of a set reducible to $B$ and a set reducible to $C$. This is not always true, as the example $A=\varnothing, B={ }^{\omega_{2}} \neq C$ shows; however, this counterexample is essentially unique.

Proposition 2.3. If $B, C \subseteq \omega_{2}$ are monotone, $A \leq_{\mathrm{m}} \mathrm{jn}^{+}(B, C)$, and $A \notin$ $\left\{\varnothing,{ }_{2}\right\}$, then $A \equiv_{\mathrm{m}} \mathrm{jn}^{+}\left(B^{\prime}, C^{\prime}\right)$ for some $B^{\prime} \leq_{\mathrm{m}} B$ and $C^{\prime} \leq_{\mathrm{m}} C$.

ProOF. First suppose $B=\varnothing$. If $C={ }^{\omega} 2$, then $A$ is clopen but not $\varnothing$ or ${ }^{\omega} 2$, so $A \equiv_{\mathrm{m}} \mathrm{jn}^{+}\left(\varnothing,{ }_{2}\right)$ (this is easy; see section III.1), so we can let $B^{\prime}=B$ and $C^{\prime}=C$; otherwise, $B \leq_{\mathrm{m}} C$, so $\mathrm{jn}^{+}(B, C) \equiv_{\mathrm{m}} C$, so we can let $B^{\prime}=B$ and $C^{\prime}=A$. The cases $B={ }^{\omega} 2, C=\varnothing$, and $C={ }^{\omega_{2}}$ are similar, so we may assume $B, C \notin\left\{\varnothing,{ }^{\omega} 2\right\}$.

Suppose $f:{ }^{\omega_{2}} \rightarrow{ }^{\omega_{2}}$ witnesses $A \leq_{\mathrm{m}} \mathrm{jn}^{+}(B, C)$. Let $B^{\prime}=A-f^{-1} " 10^{\omega} 2$ and $C^{\prime}=A-f^{-1}$ " $01^{\omega}{ }^{\omega}$. We show that these sets have the required properties by defining monotone functions witnessing the required reducibilities. To see that $B^{\prime} \leq_{\mathrm{m}} B$, define $g:{ }^{\omega} 2 \rightarrow{ }^{\omega} 2$ by

$$
g(x)= \begin{cases}y & \text { if } f(x)=01 y \\ \overline{1} & \text { if } f(x)=11 y \\ \overline{0} & \text { otherwise }\end{cases}
$$

this works since $B$ is monotone but not $\varnothing$ or ${ }^{\omega_{2}}$. We show $C^{\prime} \leq_{\mathrm{m}} C$ similarly. For $B^{\prime} \leq_{\mathrm{m}} A$, define $g(x)$ to be $x$ if $f(x)(1)=1$ and $\overline{0}$ otherwise; for $C^{\prime} \leq_{\mathrm{m}} A$, define $g(x)$ to be $x$ if $f(x)(0)=1$ and $\overline{0}$ otherwise. Finally, for $A \leq_{\mathrm{m}} \mathrm{jn}^{+}\left(B^{\prime}, C^{\prime}\right)$, define $g(x)$ to be $01 x$ if $f(x) \in 01^{\omega} 2,10 x$ if $f(x) \in 10^{\omega} 2, \overline{1}$ if $f(x) \in 11^{\omega} 2$, and $\overline{0}$ if $f(x) \in 00^{\omega}$.

An immediate consequence of this is that if the monotone degree of a set $A$ is $\mathrm{jn}^{+}$-irreducible (i.e., there are no sets $B, C<_{\mathrm{m}} A$ such that jn ${ }^{+}(B, C) \equiv_{\mathrm{m}} A$ ), then it is $\mathrm{jn}^{+}$-prime (i.e., there are no sets $B, C \geq_{\mathrm{m}} A$ such that $\mathrm{jn}^{+}(B, C) \geq_{\mathrm{m}} A$ ).

We say that two sets $X, Y \subseteq{ }^{\omega} 2$ are $m$-disjoint iff for any $x \in X$ and $y \in Y$ we have $x \not y$ and $y \leq x$. Call a set $X \subseteq{ }^{\omega} 2$ m-connected iff it cannot be expressed as the union of two nonempty m-disjoint sets, each of which is relatively closed in $X$. The m-connected component of a point $x$ in $X$ is the largest m-connected subset of $X$ containing $x$; a function $f: X \rightarrow{ }^{\omega_{2}}$ is $m$-continuous iff it is continuous and relatively monotone. The basic facts about connectedness also hold for $\mathrm{m}$ connectedness under these definitions.

It is easy to see that a monotone set $A$ whose frontier is nonempty and mconnected must be jn ${ }^{+}$-prime. (If $f$ witnesses $A \leq_{\mathrm{m}} \mathrm{jn}^{+}(B, C)$, then $f$ cannot map 
a frontier point of $A$ to $11^{\omega} 2$ or $00^{\omega} 2$; hence, $\operatorname{Fr}(A) \cap f^{-1}$ " $01^{\omega}{ }^{\omega}$ and $\operatorname{Fr}(A) \cap f^{-1}$ " $10^{\omega} 2$ form a partition of $\operatorname{Fr}(A)$ into two m-disjoint relatively closed sets, so one of them must be empty. Therefore, if $g(x)$ is $f(x)$ with the initial 01 or 10 removed, then $g$ maps $\operatorname{Fr}(A)$ into either $\operatorname{Fr}(B)$ or $\operatorname{Fr}(C)$, and we can apply Proposition 2.2 to see that $A \leq_{\mathrm{m}} B$ or $A \leq_{\mathrm{m}} C$.) The converse is not true; a simple counterexample will be given in Figure 3.2(b).

4. The 'flat' operator. In this section we will define for each set $X \subseteq{ }^{\omega_{2}}$ a set $X_{b} \subseteq{ }^{\omega} 2$; the 'flat' operator will have the following properties:

(1) $X_{\mathrm{b}}$ is monotone.

(2) $X_{b} \equiv_{w} X$.

(3) If $X$ is monotone, then $X_{b} \leq_{m} X$.

(4) If $X \leq_{\mathrm{w}} Y$, then $X_{\mathrm{b}} \leq_{\mathrm{m}} Y_{\mathrm{b}}$.

(5) $\mathrm{jn}^{+}\left(X_{\mathrm{b}}, Y_{\mathrm{b}}\right)=(\mathrm{jn}(X, Y))_{\mathrm{b}}$.

(6) $-\uparrow X_{b}=(-\Uparrow X)_{b}$.

Using these facts, we can prove several interesting properties of monotone degrees.

From (1) and (2), we see that every Wadge degree contains a monotone set. This is a nontrivial result; I believe it was first proved by C. Schlindwein and N. Kose.

Properties (1) and (4) show that the operation $X \mapsto X_{\mathrm{b}}$ is an embedding of $\left(P\left({ }^{\omega} 2\right), \leq_{\mathrm{w}}\right)$ into $\left(\right.$ Mon, $\left.\leq_{\mathrm{m}}\right)$, and $(5)$ and $(6)$ show that this embedding preserves (binary) least upper bounds and duality. (Note that (2) implies the converse of (4).) It would be nice if $(6)$ were $-\uparrow X_{b}=(-X)_{b}$, but you cannot have everything;

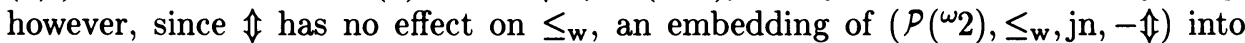
(Mon, $\leq_{\mathrm{m}}, \mathrm{jn}^{+},-\hat{\uparrow}$ ) reflects the structure we want to reflect.

Finally, (1), (2), and (4) show that the 'flat' sets lie in a single monotone degree within each Wadge degree, and (3) implies that this is the least monotone degree in the Wadge degree. (Unfortunately, it is not this easy to get greatest monotone degrees.)

We now define $X_{b}$ for each $X$. Define the function $f_{b}: \leq \omega_{2} \rightarrow \leq \omega_{2}$ by: given a finite or infinite sequence $s$, we obtain $f_{b}(s)$ by replacing each 0 in the sequence with the pair 01 and each 1 with the pair 10. (Thus, $\ell\left(f_{b}(s)\right)=2 \ell(s)$.) Then $f_{b}$ maps ${ }^{\omega_{2}}$ to a set of $\leq$-incomparable points in ${ }^{\omega_{2}}$; this means that we can define a monotone set $A$ having arbitrary intersection with $f_{b}$ " $\omega_{2}$. To get such a set which is monotone equivalent to a given set $X$, however, takes some care. Define $X_{b}$ as follows: for any $y \in{ }^{\omega_{2}}$,

$$
\begin{array}{ll}
\text { if } y=f_{\mathrm{b}}(x), & \text { then } y \in X_{\mathrm{b}} \Longleftrightarrow x \in X ; \\
\text { if } y=f_{\mathrm{b}}(s) 00 z, & \text { then } y \in X_{\mathrm{b}} \Longleftrightarrow(\forall x \supset s) x \in X ; \\
\text { if } y=f_{\mathrm{b}}(s) 11 z, & \text { then } y \in X_{\mathrm{b}} \Longleftrightarrow(\exists x \supset s) x \in X .
\end{array}
$$

(The definition of $\mathrm{jn}^{+}$can be given in a similar format. This means that the exceptional cases for $\mathrm{jn}^{+}$are not really that exceptional; in particular, it explains why there are no exceptions in property (5).)

PROOF OF (1)-(6). For (1), we must show that for any $y, y^{\prime} \in \omega^{\omega_{2}}$ with $y \leq y^{\prime}$, $y \in X_{\mathrm{b}}$ implies $y^{\prime} \in X_{\mathrm{b}}$. We will just do two cases; the rest are similar or easier. First, suppose $y=f_{\mathrm{b}}(s) 11 z$ and $y^{\prime}=f_{\mathrm{b}}\left(s^{\prime}\right) 11 z^{\prime}$. Since $y \leq y^{\prime}$, it is clear that $s$ extends $s^{\prime}$. Hence, if $y \in X_{b}$, then there is $x \in X$ extending $s$, and this $x$ also extends $s^{\prime}$, so $y^{\prime} \in X_{b}$. Next, suppose $y=f_{b}(s) 00 z$ and $y^{\prime}=f_{b}\left(s^{\prime}\right) 11 z^{\prime}$. Since 
$y \leq y^{\prime}$ and 01 is incomparable with 10 , the sequences $s$ and $s^{\prime}$ must agree as far as they are both defined; hence, there is $x \in{ }^{\omega} 2$ which extends both $s$ and $s^{\prime}$. Now $y \in X_{\mathrm{b}}$ implies $x \in X$, which implies $y^{\prime} \in X_{\mathrm{b}}$.

The map $f_{\mathrm{b}}$ witnesses $X \leq_{\mathrm{w}} X_{\mathrm{b}}$. We now construct a map $f$ witnessing $X_{\mathrm{b}} \leq_{\mathrm{w}} X$. For each $s \in{ }^{<\omega} 2$, choose points $a_{s}, b_{s} \in{ }^{\omega} 2$ such that $a_{s} \supset s, b_{s} \supset s, a_{s} \notin X$ (unless $s^{\omega} 2 \subseteq X$ ), and $b_{s} \in X$ (unless $s^{\omega} 2 \cap X=\varnothing$ ). Now define $f:{ }^{\omega} 2 \rightarrow{ }^{\omega} 2$ by: if $y=f_{b}(x)$, let $f(y)=x$; if $y=f_{\mathrm{b}}(s) 00 z$, let $f(y)=a_{s}$; if $y=f_{\mathrm{b}}(s) 11 z$, let $f(y)=b_{s}$. It is easy to see that knowing the first $2 n$ bits of $y$ (i.e., $y\lceil 2 n$ ) suffices to determine the first $n$ bits of $f(y)$, so $f$ is continuous. If $y=f_{\mathrm{b}}(x)$, then $y \in X_{\mathrm{b}}$ iff $x \in X$ iff $f(y) \in X$; if $y=f_{b}(s) 00 z$, then $y \in X_{b}$ iff $(\forall x \supset s) x \in X$ iff $a_{s} \in X$ iff $f(y) \in X$; the case $y=f_{\mathrm{b}}(s) 11 z$ is similar. Therefore, $f$ witnesses $X_{\mathrm{b}} \leq_{\mathrm{w}} X$, as desired.

The proof of (3) is the same as that of (2), except that we use the specific points $a_{s}=s \overline{0}$ and $b_{s}=s \overline{1}$ (which have the required properties since $X$ is monotone). To see that the resulting $f$ is monotone, suppose that $y, y^{\prime} \in \omega_{2}$ and $y<y^{\prime}$. Let $n$ be least such that $y(2 n) \neq y^{\prime}(2 n)$ or $y(2 n+1) \neq y^{\prime}(2 n+1)$. If a 00 or 11 occurs among the first $n$ pairs of bits in $y$ and $y^{\prime}$, then $f(y)=f\left(y^{\prime}\right)$, so we may assume $y\left\lceil 2 n=f_{b}(s)\right.$ for some $s$ of length $n$. Since $y<y^{\prime}$, either $y(2 n)=y(2 n+1)=0$ or $y^{\prime}(2 n)=y^{\prime}(2 n+1)=1$, so either $f(y)=a_{s} \leq f\left(y^{\prime}\right)$ or $f(y) \leq b_{s}=f\left(y^{\prime}\right)$.

For (4), we first note that $\operatorname{Fr}\left(X_{\mathrm{b}}\right)=f_{\mathrm{b}} " \operatorname{Fr}(X)$. (Clearly any point not in $f_{\mathrm{b}} " \omega_{2}$ has a neighborhood which either is contained in $X_{b}$ or does not meet $X_{b}$. The same applies to any point of the form $f_{b}(x)$ with $x \notin \operatorname{Fr}(X)$; e.g., if $s^{\omega} 2 \subseteq X$, then $f_{b}(s)^{\omega} 2 \subseteq X_{b}$. On the other hand, $f_{b}$ is a homeomorphism between ${ }^{\omega} 2$ and $f_{b}$ " $\omega_{2}$, so frontier points of $X$ must be mapped to frontier points of $X_{b}$.) Now, suppose $f:{ }^{\omega} 2 \rightarrow{ }^{\omega} 2$ witnesses $X \leq_{\mathrm{w}} Y$. Then the map $f_{b} \circ f \circ f_{b}{ }^{-1}: f_{b}$ " $\omega_{2} \rightarrow f_{b}$ " " $\omega_{2}$ is continuous and (vacuously) relatively monotone, and its restriction to $\operatorname{Fr}\left(X_{\mathrm{b}}\right)$ maps into $\operatorname{Fr}\left(Y_{b}\right)$ and maps points in $X$ to points in $Y$, points not in $X$ to points not in $Y$. Therefore, by Proposition $2.2, X_{\mathrm{b}} \leq_{\mathrm{m}} Y_{\mathrm{b}}$.

To prove (5) and (6), just work through the definitions.

5. The separation and reduction properties. In this section we describe two properties that are quite useful in ordinary descriptive set theory (and in particular the study of Wadge reducibility), and give analogues which are just as useful in the study of monotone reducibility.

Suppose $\Gamma$ is a collection of subsets of $\omega_{2}$. We say that the first separation property holds for $\Gamma$ (in symbols, $\operatorname{Sep}_{\mathrm{I}}(\Gamma)$ ) iff, for any disjoint $X, Y \in \Gamma$, there is $X^{\prime} \in \Gamma \cap \Gamma_{-}$such that $X \subseteq X^{\prime} \subseteq-Y$. We say that the reduction property holds for $\Gamma(\operatorname{Red}(\Gamma))$ iff, for any $X, Y \in \Gamma$, there are $X^{\prime}, Y^{\prime} \in \Gamma$ such that $X^{\prime} \subseteq X, Y^{\prime} \subseteq Y$, $X^{\prime} \cup Y^{\prime}=X \cup Y$, and $X^{\prime} \cap Y^{\prime}=\varnothing$. See Figure 2.4.

In order to motivate the monotone analogues of these two properties, we will rewrite the properties in a form that, although less symmetric, is more amenable to generalization. The first separation property for $\Gamma$ can be stated as follows: For any $X, Y \subseteq{ }^{\omega} 2$ such that $X \in \Gamma, Y \in \Gamma_{-}$, and $X \subseteq Y$, there is $X^{\prime} \in \Gamma \cap \Gamma_{-}$such that $X \subseteq X^{\prime} \subseteq Y$. And the reduction property for $\Gamma$ is equivalent to: For any $X \in \Gamma$ and $Y \in \Gamma_{-}$, there is $X^{\prime} \in \Gamma$ such that $X^{\prime} \cup Y \in \Gamma_{-}$and $X \cap Y \subseteq X^{\prime} \subseteq X$. (The $Y$ 's here are the complements of the $Y$ 's in the original statements.)

In these latter forms, the complementation operator serves only to define the dual class of the given class $\Gamma$. Let $\operatorname{Sep}_{I}^{D}(\Gamma)$ and $\operatorname{Red}^{D}(\Gamma)$ be the result of replacing - with an operator $D: P\left({ }^{\omega} 2\right) \rightarrow \mathcal{P}\left({ }^{\omega} 2\right)$. Many of the desirable properties of $\operatorname{Sep}_{\mathrm{I}}$ 


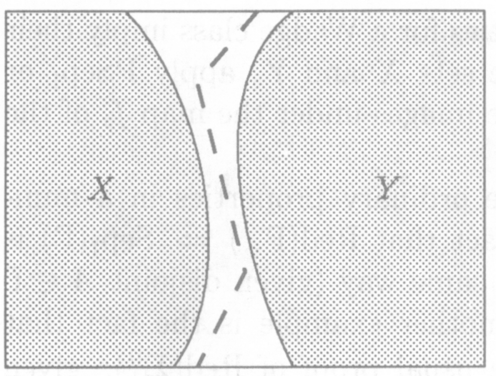

(a) First separation

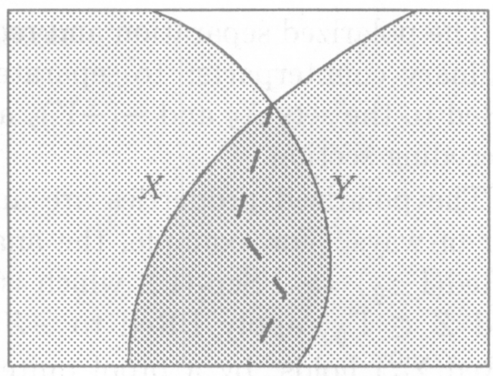

(b) Reduction

FIGURE 2.4. The separation and reduction properties

and Red will go through for $\operatorname{Sep}_{\mathrm{I}}^{D}$ and $\operatorname{Red}^{D}$ if $D$ has the duality properties of -, namely $D D X=X$ and $X \subseteq Y \Rightarrow D X \supseteq D Y$. The operator $-\mathbb{1}$ from section II. 1 has these properties and sends monotone sets to monotone sets, so $\operatorname{Sep}_{\mathrm{I}}^{-\hat{v}}$ and $\operatorname{Red}^{-1}$ are the desired monotone analogues of $\operatorname{Sep}_{\mathrm{I}}$ and Red.

When we look at $\operatorname{Sep}_{\mathrm{I}}^{-\hat{N}}(\Gamma)$ or $\operatorname{Red}^{-1}(\Gamma)$ in this paper, $\Gamma$ will usually be the collection of monotone sets in a fixed Wadge class. We therefore make the following definitions: $\operatorname{PSep}_{\mathrm{I}}(\Gamma)$ means $\operatorname{Sep}_{\mathrm{I}}^{-\uparrow}(\Gamma \cap \mathrm{Mon})$, and PRed $(\Gamma)$ means $\operatorname{Red}^{-\Uparrow}(\Gamma \cap M o n)$. The $\mathrm{P}$ stands for polarized; the motivation for this will be clear shortly.

When the definitions of PSep I and PRed are transformed into forms analogous to the original forms of $\operatorname{Sep}_{\mathrm{I}}$ and Red, they become: A class $\Gamma$ satisfies the polarized first separation property iff, for any monotone $A \in \Gamma$ and any hereditary $Z \in \Gamma_{\text {企 }}$ such that $A \cap Z=\varnothing$, there is a monotone $A^{\prime} \in \Gamma$ such that $-A^{\prime} \in \Gamma_{\hat{\forall}}$ and $A \subseteq A^{\prime} \subseteq-Z$; and $\Gamma$ satisfies the polarized reduction property iff, for any monotone $A \in \Gamma$ and any hereditary $Z \in \Gamma_{\hat{y}}$, there exist a monotone $A^{\prime} \in \Gamma$ and a hereditary $Z^{\prime} \in \Gamma_{\hat{v}}$ such that $A^{\prime} \subseteq A, Z^{\prime} \subseteq Z, A^{\prime} \cup Z^{\prime}=A \cup Z$, and $A^{\prime} \cap Z^{\prime}=\varnothing$. (See Figure 2.5.) If $\Gamma$ is a Wadge class, then $\Gamma_{\hat{v}}=\Gamma$, so we can drop the 1 's.

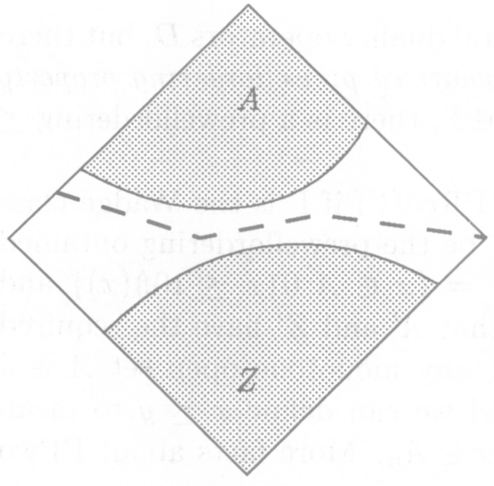

(a) Polarized first separation

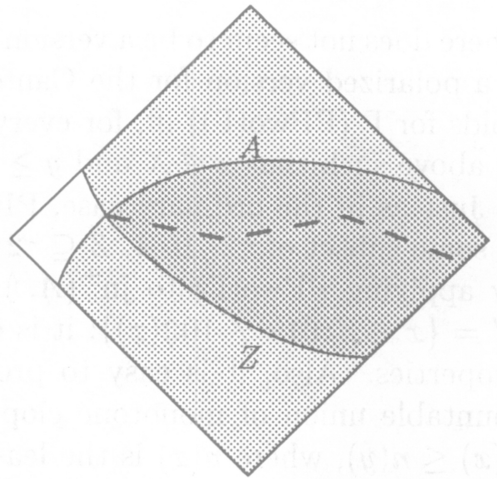

(b) Polarized reduction

FIGURE 2.5. The polarized versions 
The polarized separation and reduction properties for a Wadge class imply their ordinary counterparts: to separate or reduce the sets $X$ and $Y$, apply PSep $_{\mathrm{I}}$ or PRed to the sets $X_{b}$ and $-(-Y)_{b}$ and take inverse images under the map $f_{b}$ of the preceding section.

The polarized properties are analogous to the ordinary properties in a number of ways. For example, the analogue of the fact that $\operatorname{Red}\left(\Gamma_{-}\right) \Rightarrow \operatorname{Sep}_{\mathrm{I}}(\Gamma)$ is $\operatorname{PRed}\left(\Gamma_{-\hat{v}}\right) \Rightarrow \operatorname{PSep}_{\mathrm{I}}(\Gamma)$, which is proved in the same way (given disjoint $A \in \Gamma$ and $Z \in \Gamma_{\hat{\Downarrow}}$, apply PRed to $-Z$ and $-A$ ). Another example is the fact that $\operatorname{PRed}\left(\Sigma_{1}^{0}\right)$ holds, by a proof quite similar to the usual proof of $\operatorname{Red}\left(\Sigma_{1}^{0}\right)$ : given open sets $A$ and $Z$, apply Proposition 1.2 to $A$ and $-Z$ to get clopen sets $A_{n}$ and $Z_{n}$ such that $\bigcup_{n \in \omega} A_{n}=A$ and $\bigcup_{n \in \omega} Z_{n}=Z$; then let

$$
A^{\prime}=\bigcup_{n \in \omega}\left(A_{n}-\bigcup_{m<n} Z_{m}\right) \quad \text { and } \quad Z^{\prime}=\bigcup_{n \in \omega}\left(Z_{n}-\bigcup_{m \leq n} A_{m}\right) \text {. }
$$

This last example brings up an important point. Most proofs of $\operatorname{Sep}_{\mathrm{I}}$ or Red start by expressing the sets to be separated or reduced in a special form, which is often equivalent to being in $\mathrm{Cp}_{H}$ for some Boolean operation $H$. In this case, the proof almost always can be easily modified to give a proof of $\operatorname{Sep}_{I}^{-\hat{v}}$ or $\operatorname{Red}^{-\hat{v}}$ for the $H$-positive sets. However, we will usually be more interested in $\operatorname{PSep}_{\mathrm{I}}\left(\mathrm{Cp}_{H}\right)$ and PRed $\left(\mathrm{Cp}_{H}\right)$. This shows the importance of the equality (1.1); proofs of polarized separation and reduction will usually be easy if (1.1) holds and quite difficult otherwise.

Many classes which satisfy the reduction property also satisfy the more powerful prewellordering property. The prewellordering property holds for a collection $\Gamma$ of subsets of ${ }^{\omega_{2}}(\operatorname{Pwo}(\Gamma))$ iff, for any $X \in \Gamma$, there is a prewellordering $\preceq$ of $X$ (i.e., a reflexive, transitive, well-founded relation on $X$ such that any $x, y \in X$ satisfy either $x \preceq y$ or $y \preceq x$ ) such that the following sets are in $\Gamma$ (more precisely, their images under the canonical homeomorphism from ${ }^{\omega_{2}} \times{ }^{\omega_{2}}$ to ${ }^{\omega_{2}}$ are in $\Gamma$ ):

$$
\begin{aligned}
& \left\{(x, y) \in{ }^{\omega_{2}} \times{ }^{\omega_{2}} 2: x \in X \wedge(y \notin X \vee x \preceq y)\right\}, \\
& \left\{(x, y) \in{ }^{\omega_{2}} \times{ }^{\omega_{2}} 2: x \in X \wedge(y \notin X \vee y \npreceq x)\right\} .
\end{aligned}
$$

There does not seem to be a version of this for general duality operators $D$, but there is a polarized version for the Cantor space: the polarized prewellordering property holds for $\Gamma(\operatorname{PPwo}(\Gamma))$ iff, for every monotone $X \in \Gamma$, there is a prewellordering $\preceq$ as above such that $x \in X$ and $y \geq x$ imply $y \preceq x$.

Just as in the ordinary case, PPwo $(\Gamma)$ implies $\operatorname{PRed}(\Gamma)$ if $\Gamma$ is the Wadge class of some subset of ${ }^{\omega_{2}} 2$. If $A, Z \subseteq{ }^{\omega_{2}}$ are in $\Gamma$, let $\preceq$ be the prewellordering obtained by applying $\operatorname{PPwo}(\Gamma)$ to $\mathrm{jn}^{+}(A, \uparrow Z)$, and let $\overline{A^{\prime}}=\{x \in A: 01 x \preceq 10 \Uparrow(x)\}$ and $Z^{\prime}=\{x \in Z: 01 x \npreceq 10 \Uparrow(x)\}$; it is easy to verify that $A^{\prime}$ and $Z^{\prime}$ have the required properties. Also, it is easy to prove $\operatorname{PPwo}\left(\Sigma_{1}^{0}\right)$ : any monotone open set $A$ is a countable union of monotone clopen sets $A_{n}$, and we can define $x \preceq y$ to mean $n(x) \leq n(y)$, where $n(x)$ is the least $n$ such that $x \in A_{n}$. More facts about PPwo are given in section III.6.

We have already seen an application of PRed in the proof of Proposition 2.1. We conclude this section with an application of $\mathrm{PSep}_{\mathrm{I}}$ to give a more understandable proof of Cenzer's result [1] that every monotone $\Delta_{2}^{0}$ set $A$ is monotone reducible 
to $\left\{x \in{ }^{\omega} 2: x(n)=1\right.$ for infinitely many $\left.n\right\}$. Both $A$ and $-A$ are $\Sigma_{2}^{0}$-positive by Proposition 1.2(b), so write $A=\bigcup_{n \in \omega} A_{n}$ and $Z=\bigcup_{n \in \omega} Z_{n}$ where $A_{n}$ and $Z_{n}$ are closed; we may assume $A_{n} \subseteq A_{m}$ and $Z_{n} \subseteq Z_{m}$ for $n<m$. Apply $\operatorname{PSep}_{\mathrm{I}}\left(\boldsymbol{\Pi}_{1}^{0}\right)$ to get monotone clopen sets $C_{n}$ such that $A_{n} \subseteq C_{n} \subseteq-Z_{n}$. Now define $f:{ }^{\omega} 2 \rightarrow{ }^{\omega} 2$ by: $f(x)(n)=1$ iff $x \in C_{n}$. The function $f$ is monotone and continuous because the sets $C_{n}$ are monotone and clopen. If $x \in A$, then $x \in A_{n} \subseteq C_{n}$ and hence $f(x)(n)=1$ for sufficiently large $n$; if $x \notin A$, then $x \in Z_{n} \subseteq-C_{n}$ and hence $f(x)(n)=0$ for sufficiently large $n$. Therefore, $f$ witnesses $A \leq_{\mathrm{m}}\left\{x \in{ }^{\omega} 2: x(n)=1\right.$ for infinitely many $n\}$.

\section{SPECIFIC LEVELS IN THE WADGE HIERARCHY}

We now begin a detailed study of the monotone degrees within specific Wadge degrees. In order to do this, we start by reviewing the structure of the lower Wadge degrees. The facts here are due mainly to Hausdorff [4, esp. §30] and Wadge [14, pp. 74-92 and 141-146].

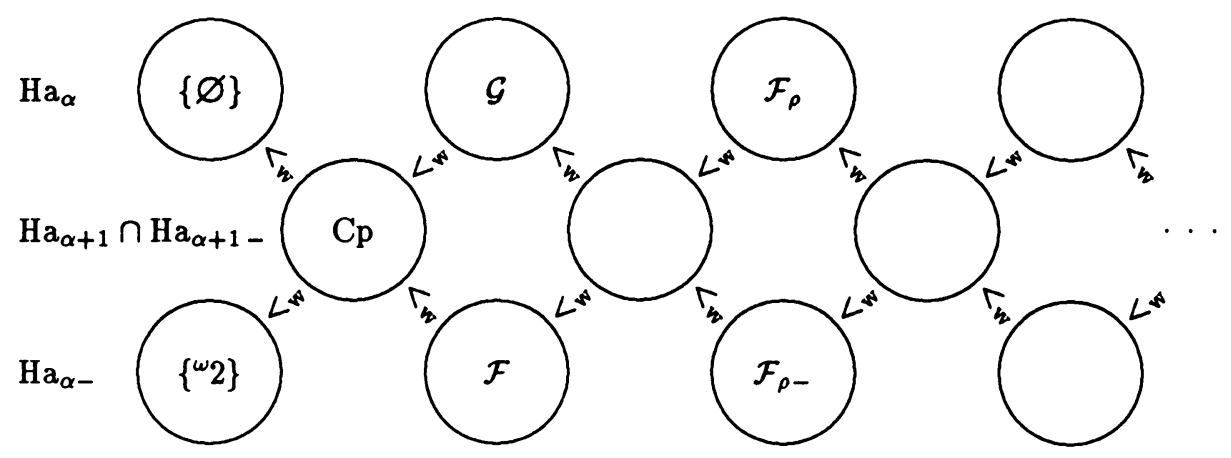

FiguRE 3.1. The lowest Wadge degrees

The Wadge degrees of $\Delta_{2}^{0}$ sets form an initial segment of the Wadge degrees; that is, any $\Delta_{2}^{0}$ set is Wadge reducible to any non- $\Delta_{2}^{0}$ set. The $\Delta_{2}^{0}$ degrees are arranged in $\omega_{1}$ levels, each containing alternately one or two degrees, as shown in Figure 3.1, where Wadge complexity increases from left to right. (Since ${ }^{\omega} 2$ is compact, the limit levels have two degrees.) The Wadge class generated by one of the degrees at the $\alpha$ 'th level with two degrees will be called $\mathrm{Ha}_{\alpha}$; the class generated by the other degree at this level will then be $\mathrm{Ha}_{\alpha-}$, and the class generated by the lone degree at the next level will be $\mathrm{Ha}_{\alpha+1} \cap \mathrm{Ha}_{\alpha+1}$. A set in the degree which generates the Wadge class $\mathrm{Ha}_{\alpha}$ will be called a strictly $\mathrm{Ha}_{\alpha}$ set; similar terminology will be used for other Wadge degrees.

Fix a set $X \subseteq{ }^{\omega_{2}}$, and define closed sets $R_{\alpha}$ for $\alpha<\omega_{1}$ as follows: $R_{0}={ }^{\omega} 2$, $R_{\lambda}=\bigcap_{\alpha<\lambda} R_{\alpha}$ for limit $\lambda$, and $R_{\alpha+1}=\overline{R_{\alpha} \cap X} \cap \overline{R_{\alpha}-X}$. Then there is a countable ordinal $\alpha_{0}$ such that $R_{\alpha}=R_{\alpha_{0}}$ for all $\alpha \geq \alpha_{0}$, and $X$ is $\Delta_{2}^{0}$ if and only if $R_{\alpha_{0}}=\varnothing$. The set $X$ is in $\mathrm{Ha}_{\alpha}$ iff $R_{\alpha} \cap X=\varnothing$, and $X$ is in $\mathrm{Ha}_{\alpha-}$ iff $R_{\alpha} \subseteq X$. An equivalent definition can be given in terms of iterated sequences of points; for example, $X$ is in $\mathrm{Ha}_{2}$ iff there do not exist points $x_{n m} \in X$ for $m, n \in \omega$ such that $\lim _{m \rightarrow \infty} x_{n m} \in{ }^{\omega} 2-X$ for each $n$ but $\lim _{n \rightarrow \infty} \lim _{m \rightarrow \infty} x_{n m} \in X$. Hence, 
$X \in \mathrm{Ha}_{2}$ iff no sequence of frontier points of $X$ which are not in $X$ converges to a point in $X$.

A few of these classes have other names, and the degrees generating these classes are marked in Figure 3.1: $\mathrm{Ha}_{0}=\{\varnothing\}, \mathrm{Ha}_{0-}=\left\{{ }^{\omega} 2\right\}, \mathrm{Ha}_{1} \cap \mathrm{Ha}_{1-}=\mathrm{Cp}, \mathrm{Ha}_{1}=\mathcal{G}$, $\mathrm{Ha}_{1-}=\mathcal{F}$, and $\mathrm{Ha}_{2}=\mathcal{F}_{\rho}$.

1. The first six Wadge degrees. The Wadge degrees $\{\varnothing\}$ and $\left\{{ }^{\omega} 2\right\}$ clearly each contain exactly one monotone degree. There is just one monotone degree of strictly clopen sets, since any monotone set in this Wadge degree can be reduced to any other by the map which sends all points in the first set to $\overline{1}$ and all other points to $\overline{0}$.

The next two Wadge degrees (the strictly open sets and the strictly closed sets) also contain exactly one monotone degree each. This fact (as well as the facts in the previous paragraph) is proven directly by Cenzer [1], but it is an immediate consequence of Proposition 2.2 (since all of the frontier points of one monotone set in the degree can be mapped to a single frontier point of another such set).

The sixth Wadge degree contains those sets $X$ such that $\operatorname{Fr}(X) \cap X$ and $\operatorname{Fr}(X)-X$ are both nonempty and closed. Among the monotone sets in this degree are the two depicted in Figure 2.2 (call them $A$ and $B$, respectively); it is clear that $A<_{\mathrm{m}} B$. It turns out that these two sets represent the only two monotone degrees in this Wadge degree; Proposition 2.2 implies that any monotone set in this degree having a frontier point in the set lying above a frontier point outside the set is monotone equivalent to $B$, while any other monotone set in the degree is monotone equivalent to $A$.

We will now see that the simplicity of these first six degrees stops abruptly at the degree of strictly $\mathcal{F}_{\rho}$ sets.

2. Differences of closed sets. The main purpose of this long section is to show that the collection of $\mathcal{F}_{\rho}$ monotone degrees is very large and complicated, containing, among other things, uncountable ascending and descending chains and antichains.

2.1. A few examples. Figure 3.2 shows seven monotone-inequivalent $\mathcal{F}_{\rho}$ sets; it gives some idea of the possibilities available at this level. (The outer diamond is omitted in the second row because it just gets in the way.) Recall that a set is strictly $\mathcal{F}_{\rho}$ iff there is a sequence of frontier points in the set converging to a frontier point outside the set, but not vice versa.

Diagram (a) is supposed to represent the most natural monotone $\mathcal{F}_{\rho}$ set, namely the truth table of the Boolean operation $\delta \cap \sigma$, which can be written as $\{x \in$ ${ }^{\omega_{2}} 2: \forall n x(2 n)=1$ and $\left.\exists n x(2 n+1)=1\right\}$. If $A \subseteq{ }^{\omega_{2}}$ is monotone and $\mathcal{F}_{\rho}$, then $\bar{A}-A$ is closed and relatively hereditary in $\bar{A}$, so $A=\bar{A} \cap-(\bar{A}-A)_{\leq}$, which means that $A$ is the intersection of a monotone closed set and a monotone open set. Hence, $A$ is in $(\mathrm{Cp} \cap \mathrm{Mon})_{\delta \cap \sigma}$, which proves (1.1) for $\delta \cap \sigma$ and shows that the monotone degree of (a) is the greatest $\mathcal{F}_{\rho}$ monotone degree.

Since we have (1.1) for $\delta \cap \sigma$, it is reasonable to suppose that the usual proof of $\operatorname{Red}\left(\mathcal{F}_{\rho}\right)$ can be transformed into a proof of $\operatorname{PRed}\left(\mathcal{F}_{\rho}\right)$, and this is indeed the case. Given $\mathcal{F}_{\rho}$ sets $A$ and $Z$, write $A=A_{0} \cap A_{1}$ and $Z=Z_{0} \cap Z_{1}$ where $A_{0}$ and $Z_{0}$ are closed and $A_{1}$ and $Z_{1}$ are open. Apply $\operatorname{PRed}(\mathcal{G})$ to $A_{1}$ and $Z_{1}$ to get $A_{1}^{\prime}$ and $Z_{1}^{\prime}$, 


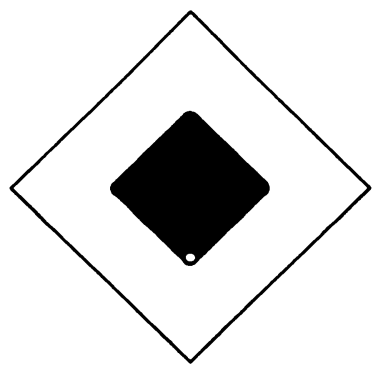

(a)

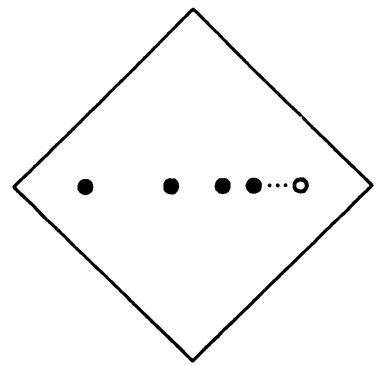

(b)

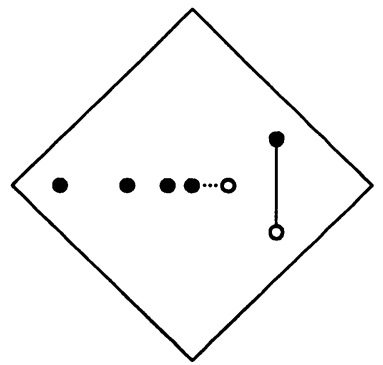

(c)

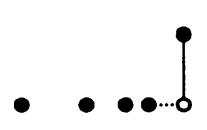

(d)

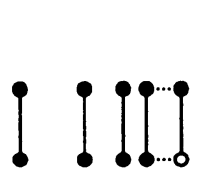

(e)

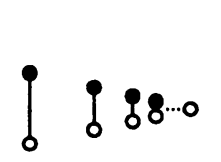

(f)

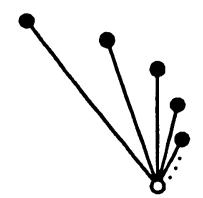

(g)

FigURE 3.2. Examples of $\mathcal{F}_{\rho}$ monotone sets

and let $A^{\prime}=A_{0} \cap\left(A_{1}^{\prime} \cup\left(A_{1}-Z_{0}\right)\right)$ and $Z^{\prime}=Z_{0} \cap\left(Z_{1}^{\prime} \cup\left(Z_{1}-A_{0}\right)\right)$; it is easy to verify that $A^{\prime}$ and $Z^{\prime}$ reduce $A$ and $Z$ as desired.

The set in (a) has a sequence of frontier points $x_{n}$ such that $x_{n}$ is in the set for each $n, x_{0}>x_{1}>\cdots$, and $\lim _{n \rightarrow \infty} x_{n}$ is not in the set; hence, any set to which the set in (a) is monotone reducible must have such a sequence. Conversely, the set $\left\{x \in{ }^{\omega} 2: \forall n x(2 n)=1\right.$ and $\left.\exists n x(2 n+1)=1\right\}$ is monotone reducible to any monotone set having such a sequence (map points in the set which begin with $(10)^{n} 11$ to $x_{n}$ ), so any such set which is $\mathcal{F}_{\rho}$ is in the greatest $\mathcal{F}_{\rho}$ monotone degree. Note that any monotone set having such a sequence must have uncountably many frontier points, since the limit point and the first point in the sequence differ at infinitely many coordinates and any point between these two must be a frontier point.

It is easy to see that the set in (b) is monotone reducible to any strictly $\mathcal{F}_{\rho}$ monotone set, so it is in the least strictly $\mathcal{F}_{\rho}$ monotone degree (i.e., the one containing $X_{b}$ for strictly $\left.\mathcal{F}_{\rho} X\right)$. The set in (c) is the monotone join of the sets in (b) and in 2.2(b).

The rest of the diagrams show various possibilities for putting together convergent sequences of points to form frontiers of monotone $\mathcal{F}_{\rho}$ sets. (For those who need to be convinced that these diagrams can be embedded in ${ }^{\omega} 2$, one way of placing the isolated frontier points is as follows: for (e), $0(01)^{n} \overline{10}$ and $1(01)^{n} \overline{10}$; for (f), $(01)^{n} 100 \overline{01}$ and $(01)^{n} 101 \overline{01}$; for $(\mathrm{g}),(01)^{n} 11 \overline{01}$.) It is easy to verify that no two of (a)-(g) are monotone equivalent. This ad hoc list of configurations could be continued indefinitely; in the next two subsections we will proceed more systematically.

2.2. Bushes. In this section we will generalize the example in Figure 3.2(g) to obtain a large collection of $\mathcal{F}_{\rho}$ monotone degrees including, among other things, an increasing sequence of degrees of length $\omega_{1}$, and chains and antichains of cardinality $2^{\aleph_{0}}$. Let $[\omega]^{<\omega}$ denote the collection of finite subsets of $\omega$. 
DEFINITION. A bush is a nonempty set $S \subset[\omega]^{<\omega}$ such that any subset of a member of $S$ is in $S$ and $\langle S, \supset\rangle$ is well-founded. Given a bush $S$, let

$$
B(S)=\left\{x \in{ }^{\omega} 2: x \neq \overline{10} \text { and } \neg \exists m \in \omega[x(2 m)=0 \wedge\{n<m: x(2 n+1)=1\} \in S]\right\} .
$$

Also, for any bush $S$, define a function $\mathrm{rk}_{S}: S \rightarrow \omega_{1}$ by recursion on $\supset$ as follows: for any $u \in S, \operatorname{rk}_{S}(u)$ is the least ordinal $\alpha$ such that $\alpha>\operatorname{rk}_{S}(v)$ for all but finitely many $v \supset u$ in $S$.

Note that $u \subset v \in S$ implies $\operatorname{rk}_{S}(u) \geq \operatorname{rk}_{S}(v)$. For any $u \subseteq \omega$, let $\xi(u)$ be the characteristic function of $\{2 n: n \in \omega\} \cup\{2 n+1: n \in u\}$.

PROPOSITION 3.1. For any bush $S, B(S)$ is monotone; if $\mathrm{rk}_{S}(\varnothing)>0$, then $B(S)$ is strictly $\mathcal{F}_{\rho}$. The frontier of $B(S)$ is $\{\xi(u): u \in S\}$; the only frontier point of $B(S)$ which is not in $B(S)$ is $\overline{10}$.

PROOF. If $x<\overline{10}$, then $x(2 m)=0$ for some $m$, and since $\varnothing \in S$ we see that $x \notin B(S)$. It follows easily from the fact that every subset of a member of $S$ is in $S$ that $B(S) \cup\{\overline{10}\}$ is monotone, so $B(S)$ is monotone. It also follows from this fact that, if $x(2 m)=0$ for some $m$, then membership of $x$ in $B(S)$ is determined by $x \uparrow(2 m+1)$, so $x \notin \operatorname{Fr}(B(S))$. If $x=\xi(u)$ where $u \notin S$, then $u \cap n \notin S$ for some $n$ since $S$ is well-founded under $\supset$, and this implies $y \in B(S)$ for any $y \in{ }^{\omega_{2}}$ such that $y\lceil 2 n=x\lceil 2 n$. Therefore, $\operatorname{Fr}(B(S)) \subseteq\{\xi(u): u \in S\}$. On the other hand, it is easy to see that, if $x=\xi(u)$ and $u \in S$, then $(x\lceil n) \overline{1} \in B(S)$ and $(x \mid n) \overline{0} \notin B(S)$ for any $n$, so $x \in \operatorname{Fr}(B(S))$. Clearly the only frontier point of $B(S)$ not in $B(S)$ is $\overline{10}=\xi(\varnothing)$, so $\overline{B(S)}-B(S)$ is closed, so $B(S)$ is $\mathcal{F}_{\rho}$. Finally, suppose $\operatorname{rk}_{S}(\varnothing)>0$. Then $S$ is infinite, so $\bigcup S$ must be infinite. For each $n \in \bigcup S,\{n\}$ is a subset of a member of $S$ and is therefore itself a member of $S$. This means that $\xi(\{n\}) \in B(S) \cap \operatorname{Fr}(B(S))$ for arbitrarily large $n$; since $\lim _{n \rightarrow \infty} \xi(\{n\})=\overline{10} \notin B(S), B(S)$ is strictly $\mathcal{F}_{\rho}$.

The proof that $\operatorname{rk}_{S}(\varnothing)>0$ implies $\{n\} \in S$ for infinitely many $n$ can easily be extended to show that $\operatorname{rk}_{S}(u)>\alpha$ implies $\operatorname{rk}_{S}(u \cup\{n\}) \geq \alpha$ for infinitely many $n$ (use the fact that $\left\{v \supset u: \operatorname{rk}_{S}(v) \geq \alpha\right\}$ is infinite).

Proposition 3.2. If $S$ and $T$ are bushes, the function $f: \omega_{2} \rightarrow \omega_{2}$ witnesses $B(S) \leq_{\mathrm{m}} B(T)$, and $u \in S$, then $f(\xi(u))$ is $\xi(v)$ for some $v \in T$, and $\operatorname{rk}_{S}(u) \leq$ $\operatorname{rk}_{T}(v)$.

PROOF. Since $f$ must map frontier points to frontier points, it is clear that $f(\xi(u))$ is $\xi(v)$ for some (unique) $v \in T$. We prove $\operatorname{rk}_{S}(u) \leq \operatorname{rk}_{T}(v)$ by $\supset$-induction on $u$. So suppose the proposition is true for all $u^{\prime} \supset u$ in $S$. If $\operatorname{rk}_{S}(u)=0$, we are done. Otherwise, there is a sequence $\left\langle u_{n}: n \in \omega\right\rangle$ such that $u_{n} \supset u, u_{n} \in S$, $\operatorname{rk}_{S}\left(u_{n}\right) \leq \operatorname{rk}_{S}\left(u_{n+1}\right), u_{n} \neq u_{m}$ for $n \neq m$, and $\operatorname{rk}_{S}(u)$ is the proper supremum of $\left\{\operatorname{rk}_{S}\left(u_{n}\right): n \in \omega\right\}$. Let $v_{n}=\xi^{-1}\left(f\left(\xi\left(u_{n}\right)\right)\right)$; then $v_{n} \in T, v_{n} \supseteq v$ (since $f$ is monotone), and $\operatorname{rk}_{T}\left(v_{n}\right) \geq \operatorname{rk}_{S}\left(u_{n}\right)$. We will show that $\left\{v_{n}: n \in \omega\right\}$ is infinite; this will imply $\operatorname{rk}_{T}(v) \geq \operatorname{rk}_{S}(u)$, as desired. Since $\left\{u_{n}: n \in \omega\right\}$ is infinite, $\bigcup_{n \in \omega} u_{n}$ is infinite. Let $k_{0}<k_{1}<\cdots$ enumerate $\bigcup_{n \in \omega} u_{n}$. For each $i \in \omega,\left\{k_{i}\right\} \subseteq u_{n}$ for some $n$, so $\xi^{-1}\left(f\left(\xi\left(\left\{k_{i}\right\}\right)\right)\right) \subseteq v_{n}$ for that $n$. Furthermore, $f\left(\xi\left(\left\{k_{i}\right\}\right)\right) \in B(T)$ but $\lim _{i \rightarrow \infty} f\left(\xi\left(\left\{k_{i}\right\}\right)\right)=f\left(\lim _{i \rightarrow \infty} \xi\left(\left\{k_{i}\right\}\right)\right)=f(\overline{10}) \notin B(T)$, so $\left\{f\left(\xi\left(\left\{k_{i}\right\}\right)\right): i \in \omega\right\}$ is infinite, so $\left\{v^{\prime}: \exists n v^{\prime} \subseteq v_{n}\right\}$ is infinite; since each $v_{n}$ is finite, $\left\{v_{n}: n \in \omega\right\}$ is infinite. 
COROLlARY. If $S, T$, and $f$ are as above, then $\operatorname{rk}_{S}(\varnothing) \leq \mathrm{rk}_{T}(\varnothing)$; also, if $\operatorname{rk}_{S}(u) \geq \alpha$ for some nonempty $u \in S$, then $\operatorname{rk}_{T}(v) \geq \alpha$ for some nonempty $v \in T$.

Now for some more definitions. For any $g: \omega \rightarrow \omega$ and any $S \subseteq[\omega]^{<\omega}$, let $g$ " " $S=\{g$ " $u: u \in S\}$. Given $S \subseteq[\omega]^{<\omega}$, let $J_{1}(S)=\left(g_{+}\right.$" " $\left.S\right) \cup\{v \cup\{0\}: v \in$ $g_{+}$" " $\left.S\right\}$, where $g_{+}(n)=n+1$ for $n \in \omega$. Let $J_{2}\left(S, S^{\prime}\right)=\left(g_{e}\right.$ " " $\left.S\right) \cup\left(g_{o}\right.$ " " $\left.S^{\prime}\right)$, where $g_{e}(n)=2 n$ and $g_{o}(n)=2 n+1$. Fix a bijective pairing function $j: \omega \times \omega \rightarrow \omega$ which is strictly increasing in each argument, and define $J_{\omega}:{ }^{\omega} \mathcal{P}\left([\omega]^{<\omega}\right) \rightarrow \mathcal{P}\left([\omega]^{<\omega}\right)$ by: given $S_{n} \subseteq[\omega]^{<\omega}$ for $n \in \omega$, let $J_{\omega}\left(\left\langle S_{n}: n \in \omega\right\rangle\right)=\bigcup_{n \in \omega} g_{n}$ " " $S_{n}$, where $g_{n}(m)=j(n, m)$ for $n, m \in \omega$. Define $R_{\alpha}$ and $R_{\alpha}^{\prime}$ for $\alpha<\omega_{1}$ as follows: Let $R_{0}=\{\varnothing\}$. Given $R_{\alpha}$, let $R_{\alpha}^{\prime}=J_{1}\left(R_{\alpha}\right)$ and $R_{\alpha+1}=J_{\omega}\left(\left\langle R_{\alpha}^{\prime}: n \in \omega\right\rangle\right)$. Finally, for a countable limit ordinal $\lambda$, choose a sequence $\alpha_{0}<\alpha_{1}<\cdots$ with limit $\lambda$, and let $R_{\lambda}=J_{\omega}\left(\left\langle R_{\alpha_{n}}^{\prime}: n \in \omega\right\rangle\right)$. (The sets $R_{\alpha}$ and $R_{\alpha}^{\prime}$ are not well-defined because of the need to choose cofinal sequences in limit ordinals, but we will see that the monotone degrees of $B\left(R_{\alpha}\right)$ and $B\left(R_{\alpha}^{\prime}\right)$ are well-defined.)

Since the functions $g_{+}, g_{e}, g_{o}$, and $g_{n}$ are all one-to-one, we easily see that $J_{1}, J_{2}$, and $J_{\omega}$ map bushes to bushes; hence, $R_{\alpha}$ and $R_{\alpha}^{\prime}$ are bushes for all $\alpha$. Note that the frontier of $B\left(R_{1}\right)$ is isomorphic to Figure $3.2(\mathrm{~g})$. Also note that $\{0\} \in R_{\alpha}$ for all $\alpha>0$, since $\{0\} \in R_{\alpha}^{\prime}$ for all $\alpha$ and $g_{e}(0)=j(0,0)=0$.

\section{PROPOSITION 3.3. For all $\alpha<\omega_{1}$,}

$$
\operatorname{rk}_{R_{\alpha}}(\varnothing)=\operatorname{rk}_{R_{\alpha}^{\prime}}(\varnothing)=\operatorname{rk}_{R_{\alpha}^{\prime}}(\{0\})=\alpha .
$$

ProOF. An easy J-induction on $u$ shows that, if $S$ is a bush, $g: \omega \rightarrow \omega$ is oneto-one, and $u \in S$, then $\operatorname{rk}_{S}(u)=\operatorname{rk}_{g}$ "' $S(g$ " $u)$. Similarly, we see that: if $u \in S$, then $\operatorname{rk}_{J_{1}(S)}\left(g_{+} " u\right)=\operatorname{rk}_{J_{1}(S)}\left(\{0\} \cup g_{+} " u\right)=\operatorname{rk}_{S}(u)$; if $u \in S$ and $u \neq \varnothing$, then $\operatorname{rk}_{J_{2}\left(S, S^{\prime}\right)}\left(g_{e} " u\right)=\operatorname{rk}_{J_{2}\left(S^{\prime}, S\right)}\left(g_{o} " u\right)=\operatorname{rk}_{S}(u)$; and if $u \in S_{k}$ and $u \neq \varnothing$, then $\operatorname{rk}_{J_{\omega}\left(\left\langle S_{n}: n \in \omega\right\rangle\right)}\left(g_{k} " u\right)=\operatorname{rk}_{S_{k}}(u)$. The proposition now follows by a straightforward induction on $\alpha$.

PROPOSITION 3.4. If $S$ is a bush and $\operatorname{rk}_{S}(\varnothing) \geq \alpha$, then there is a strictly increasing function $h: \omega \rightarrow \omega$ such that $h$ " " $R_{\alpha} \subseteq S$. If $\operatorname{rk}_{S}(u) \geq \alpha$ for some nonempty $u$, then there is a strictly increasing function $h: \omega \rightarrow \omega$ such that $h$ " " $R_{\alpha}^{\prime}$ $\subseteq S$

Proof. Define functions $t_{\alpha}, t_{\alpha}^{\prime}: \omega \rightarrow[\omega]^{<\omega}$ as follows: Let $t_{0}(n)=\varnothing$. Given $t_{\alpha}$, let $t_{\alpha}^{\prime}(0)=\{0\}$ and, for each $n$, let $t_{\alpha}^{\prime}(n+1)=\varnothing$ if $t_{\alpha}(n)=\varnothing$; otherwise, let $t_{\alpha}^{\prime}(n+1)=\{0\} \cup g_{+} " t_{\alpha}(n)$. Given $t_{\alpha}^{\prime}$, let $t_{\alpha+1}(j(n, m))=g_{n}$ " $t_{\alpha}^{\prime}(m)$ for $n, m \in \omega$. Finally, if $\lambda$ is a limit ordinal and $\left\langle\alpha_{n}: n \in \omega\right\rangle$ is the sequence chosen in the definition of $R_{\lambda}$, let $t_{\lambda}(j(n, m))=g_{n} " t_{\alpha_{n}}^{\prime}(m)$.

It is not hard to verify the following facts by induction on $\alpha$. For any nonempty $u \in[\omega]^{<\omega}, u \in R_{\alpha}$ iff $u \subseteq t_{\alpha}(\max (u))$, and $u \in R_{\alpha}^{\prime}$ iff $u \subseteq t_{\alpha}^{\prime}(\max (u))$. If $t_{\alpha}(n) \neq \varnothing$, then $\max \left(t_{\alpha}(n)\right)=n$; if $t_{\alpha}^{\prime}(n) \neq \varnothing$, then $\max \left(t_{\alpha}^{\prime}(n)\right)=n$. If $m \in t_{\alpha}(n)$, then $t_{\alpha}(m)=t_{\alpha}(n) \cap(m+1)$; similarly for $t_{\alpha}^{\prime}$. If $u \in R_{\alpha}-\{\varnothing\}$, then $\operatorname{rk}_{R_{\alpha}}(u)=$ $\mathrm{rk}_{R_{\alpha}}\left(t_{\alpha}(\max (u))\right)$; similarly for $R_{\alpha}^{\prime}$ and $t_{\alpha}^{\prime}$. If $u \in R_{\alpha}-\{\varnothing\}$ and $\mathrm{rk}_{R_{\alpha}}(u)=0$, then there is no $v \in R_{\alpha}$ such that $v \supset t_{\alpha}(\max (u))$. If $u \in R_{\alpha}-\{\varnothing\}$ and $\operatorname{rk}_{R_{\alpha}}(u)>0$, then there are infinitely many $n>\max (u)$ such that $t_{\alpha}(n)=t_{\alpha}(\max (u)) \cup\{n\}$; $\mathrm{rk}_{R_{\alpha}}(u \cup\{n\}$ ) for such $n$ increases (not necessarily strictly) as $n$ increases, and the least ordinal greater than $\operatorname{rk}_{R_{\alpha}}(u \cup\{n\})$ for all such $n$ is $\operatorname{rk}_{R_{\alpha}}(u)$. Analogous 
statements hold for $R_{\alpha}^{\prime}$ and $t_{\alpha}^{\prime}$. The same statements hold for $u=\varnothing$ if we replace $t_{\alpha}(\max (u))$ with $\varnothing, t_{\alpha}^{\prime}(\max (u))$ with $\{0\}$, and " $n>\max (u)$ " with " $n \geq 0$ " (for $R_{\alpha}$ ) or " $n>0$ " (for $R_{\alpha}^{\prime}$ ).

Using these facts, we now define $h$ for $R_{\alpha}$ as follows: If $\alpha=0$, let $h(0)=0$; otherwise, let $h(0)$ be the least $k$ such that $\mathrm{rk}_{S}(\{k\}) \geq \mathrm{rk}_{R_{\alpha}}(\{0\})$. Now, suppose $h(i)$ has been defined for $i \leq n$ so that $\operatorname{rk}_{S}\left(h\right.$ " $\left.t_{\alpha}(i)\right) \geq \operatorname{rk}_{R_{\alpha}}\left(t_{\alpha}(i)\right)$ for $i \leq n$. If $\{n+1\} \notin R_{\alpha}$, let $h(n+1)=h(n)+1$; otherwise, let $h(n+1)$ be the least $k>h(n)$ such that $\operatorname{rk}_{S}\left(\{k\} \cup h "\left(t_{\alpha}(n+1)-\{n+1\}\right)\right) \geq \operatorname{rk}_{R_{\alpha}}\left(t_{\alpha}(n+1)\right)$. The definition of $h$ for $R_{\alpha}^{\prime}$ is analogous, but no exception is made for $\alpha=0$.

Proposition 3.5. For any bushes $S$ and $S^{\prime}, B(S) \leq_{\mathrm{m}} B\left(S^{\prime}\right)$ iff there is a function $h: \omega \rightarrow \omega$ such that $h^{-1}$ " $\{k\}$ is finite for all $k \in \omega$ and $h$ " " $S \subseteq S^{\prime}$.

ProOF. First, suppose $f:{ }^{\omega_{2}} \rightarrow{ }^{\omega_{2}}$ witnesses $B(S) \leq_{\mathrm{m}} B\left(S^{\prime}\right)$. Define $h$ as follows: if $\{n\} \in S$, let $h(n)$ be the least member of $\xi^{-1}(f(\xi(\{n\})))$ (which must exist because $f(\xi(\{n\}))$ must be a member of $\left.\operatorname{Fr}\left(B\left(S^{\prime}\right)\right) \cap B\left(S^{\prime}\right)\right)$; otherwise, let $h(n)=n$. For each $u \in S$ and each $n \in u, \xi^{-1}(f(\xi(\{n\}))) \subseteq \xi^{-1}(f(\xi(u)))$ since $f$ is monotone; therefore, $h$ " $u \subseteq \xi^{-1}(f(\xi(u))) \in S^{\prime}$, so $h$ " $u \in S^{\prime}$. Also, since $f$ is continuous, $\lim _{n \rightarrow \infty} f(\xi(\{n\}))=f\left(\lim _{n \rightarrow \infty} \xi(\{n\})\right)=f(\overline{10})=\overline{10}$, so for any $k \in \omega, f(\xi(\{n\}))(2 k+1)=1$ for only finitely many $n$; since $n \neq k$ and $h(n)=k$ imply $f(\xi(\{n\}))(2 k+1)=1, h^{-1}$ " $\{k\}$ is finite.

Conversely, suppose we have $h$ with the above properties, and define $f: \operatorname{Fr}(B(S))$ $\rightarrow{ }^{\omega} 2$ by $f(x)=\xi\left(h^{\text {" }} \xi^{-1}(x)\right)$. Since $h$ " " $S \subseteq S^{\prime}, f$ maps $\operatorname{Fr}(B(S))$ into $\operatorname{Fr}\left(B\left(S^{\prime}\right)\right)$. Clearly $f$ is relatively monotone and $f(x)=\overline{10}$ iff $x=\overline{10}$. To see that $f$ is continuous, suppose $u_{m} \in S$ for $m \in \omega$ and $\lim _{m \rightarrow \infty} \xi\left(u_{m}\right)=\xi(u)$; this means that each $n \in u$ is in $u_{m}$ for all but finitely many $m$, while each $n \notin u$ is in $u_{m}$ for only finitely many $m$. Clearly $f\left(\xi\left(u_{m}\right)\right)(2 k)=f(\xi(u))(2 k)=1$ for all $m$ and $k$. If $f(\xi(u))(2 k+1)=1$, then $h(n)=k$ for some $n \in u$, and this $n$ will be in $u_{m}$ for all but finitely many $m$, so $f\left(\xi\left(u_{m}\right)\right)(2 k+1)=1$ for all but finitely many $m$. If $f(\xi(u))(2 k+1)=0$, then there are only finitely many $n$ such that $h(n)=k$, and each of these $n$ is in $u_{m}$ for only finitely many $m$ since it is not in $u$, so $f\left(\xi\left(u_{m}\right)\right)(2 k+1)=0$ for all but finitely many $m$. Therefore, $\lim _{m \rightarrow \infty} f\left(\xi\left(u_{m}\right)\right)=f(\xi(u))$, as desired.

It follows easily that the monotone degrees of $B\left(R_{\alpha}\right)$ and $B\left(R_{\alpha}^{\prime}\right)$ do not depend on the choices of cofinal sequences in countable limit ordinals (or on the choice of the pairing function $j$ ). Also, for any $\alpha<\omega_{1}$ and any bush $S, B\left(R_{\alpha}\right) \leq_{\mathrm{m}} B(S)$ iff $\operatorname{rk}_{S}(\varnothing) \geq \alpha$, and $B\left(R_{\alpha}^{\prime}\right) \leq_{\mathrm{m}} B(S)$ iff $\operatorname{rk}_{S}(u) \geq \alpha$ for some nonempty $u \in S$. In particular, $B\left(R_{\alpha}\right)<_{\mathrm{m}} B\left(R_{\alpha}^{\prime}\right)<_{\mathrm{m}} B\left(R_{\beta}\right)$ for $\alpha<\beta<\omega_{1}$, so we have an increasing $\omega_{1}$-sequence of $\mathcal{F}_{\rho}$ monotone degrees.

Many other monotone degrees can be constructed by using 'denser' bushes such as $\{u \subset \omega:|u| \leq N\}$ for some fixed $N \in \omega$, along with the combining operations $J_{1}, J_{2}$, and $J_{\omega}$. We will conclude this subsection by looking at the bushes containing only sets of cardinality at most 2 , and showing that even at this low level a great many monotone degrees can be constructed.

A bush containing only sets of cardinality at most 2 can be viewed as a countable (simple) graph, whose vertices are the sets of cardinality 1 and whose edges are the sets of cardinality 2 . The preceding proposition implies that the monotone set associated with graph $G_{0}$ is monotone reducible to that associated with $G_{1}$ iff there 
is a function mapping the vertices of $G_{0}$ to those of $G_{1}$ such that adjacent vertices are mapped to adjacent or equal vertices and any vertex of $G_{1}$ has only finitely many preimages in $G_{0}$. In particular, the monotone degree of the set associated with $G$ does not depend on how the vertices of $G$ are identified with one-element subsets of $\omega$.

We will construct connected graphs $G_{n}$ for $n \in \omega$ such that a function mapping $G_{n}$ into $G_{n^{\prime}}$ as above exists iff $n=n^{\prime}$. Given this, consider, for each $z \subseteq \omega$, the graph $G(z)$ which is the disjoint union of $G_{n}$ for $n \in z$. Since the graphs $G_{n}$ are connected, a map from $G(z)$ to $G\left(z^{\prime}\right)$ as above must take all vertices of a given $G_{n}$ to a single $G_{m}$; hence, it is easy to see that $G(z)$ can be mapped into $G\left(z^{\prime}\right)$ in this way iff $z \subseteq z^{\prime}$. Therefore, if $A(z)$ is the monotone set associated with $G(z)$, then $A(z) \leq_{\mathrm{m}} A\left(z^{\prime}\right)$ iff $z \subseteq z^{\prime}$. We therefore have an embedding of $(\mathcal{P}(\omega), \subseteq)$ (or, equivalently, $\left.\left({ }^{\omega} 2, \leq\right)\right)$ into the $\mathcal{F}_{\rho}$ monotone degrees. In particular, this gives an antichain of size $2^{\aleph_{0}}$ (namely $A\left(\left\{n: f_{b}(x)(n)=1\right\}\right.$ ) for $x \in{ }^{\omega} 2$, where $f_{b}$ is as defined in section II.4) and a chain whose order type is that of the real numbers (namely $A(\{n: g(n)<r\})$ for $r \in \mathbf{R}$, where $g$ maps $\omega$ onto the set of rational numbers).

We start by constructing smaller graphs which will be combined to form $G_{n}$. Let $G_{A}$ be the graph with vertices $a$ and $b_{m}$ for $m \in \omega$ and edges $\left(a, b_{m}\right)$ and $\left(b_{m}, b_{m+1}\right)$ $(m \in \omega)$. If a function $f$ maps (the vertices of) $G_{A}$ into another graph as above, then $f(a) \neq f\left(b_{m}\right)$ for sufficiently large $m$ and $f\left(b_{m}\right) \neq f\left(b_{m+1}\right)$ for arbitrarily large $m$, so there is an $m$ such that $f(a), f\left(b_{m}\right)$, and $f\left(b_{m+1}\right)$ are distinct. Then these three vertices must form a triangle in the range graph, and $f(a)$ must have infinitely many neighbors since $a$ does.

Now fix $n \in \omega$, and let $D_{n}$ be a graph with vertices $d_{s}$ for all sequences $s$ of natural numbers such that $\ell(s) \leq n$ whose edges are those pairs of the form $\left(d_{s}, d_{s k}\right)$ for $k \in \omega$ and $s$ a sequence of length less than $n$. Suppose a function $f$ maps $D_{n}$ into $D_{n^{\prime}}$ as above. We will write $d_{s}^{\prime}$ to denote a vertex of $D_{n^{\prime}}$. Suppose $f\left(d_{\langle\rangle}\right)=d_{t}^{\prime}$. Only finitely many neighbors of $d_{\langle\rangle}$can be mapped to the points $d_{t}^{\prime}$ and $d_{t(\ell(t)-1)}^{\prime}$, so there must exist $i_{0}, j_{0} \in \omega$ such that $\left.f\left(d_{i_{0}}\right)=d_{t j_{0}}^{\prime}\right)$. Similarly, there must be $i_{1}$ and $j_{1}$ such that $f\left(d_{i_{0} i_{1}}\right)=d_{t j_{0} j_{1}}^{\prime}$, and repeating for $n$ steps we find $i_{0}, i_{1}, \ldots, i_{n-1}$ and $j_{0}, j_{1}, \ldots, j_{n-1}$ such that $f\left(d_{i_{0} i_{1} \cdots i_{n-1}}\right)=d_{t j_{0} j_{1} \cdots j_{n-1}}^{\prime}$. This implies $\ell\left(t j_{0} j_{1} \cdots j_{n-1}\right) \leq n^{\prime}$, so $n \leq n^{\prime}$.

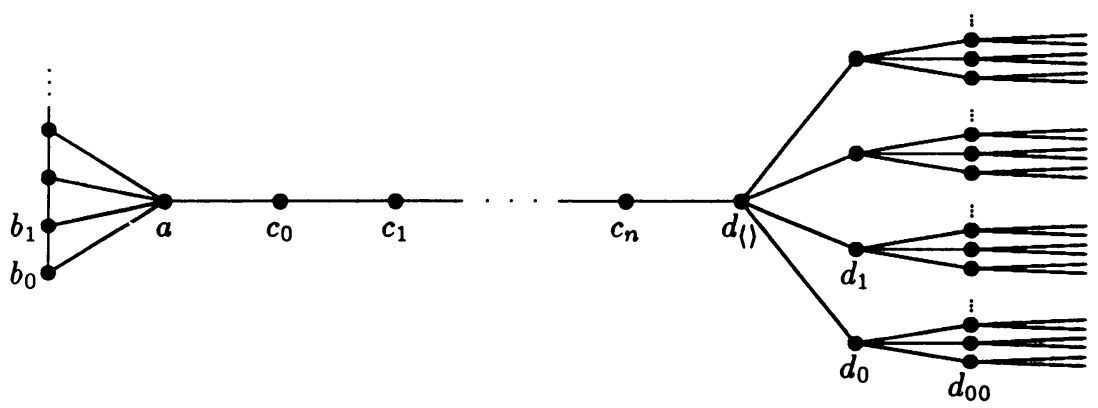

FigURE 3.3. The graph $G_{n}$ 
Now define $G_{n}$ to be $G_{A}$ joined to $D_{n+2}$ by a path of length $n+2$ with vertices $a$, $c_{0}, c_{1}, \ldots, c_{n}$, and $d_{\langle\rangle}$, as shown in Figure 3.3. Suppose $f$ maps the vertices of $G_{n}$ to those of $G_{n^{\prime}}$ (where we will put primes on the latter as before), $f$ maps adjacent vertices to adjacent or equal vertices, and the preimage under $f$ of a finite set is finite. Then $f(a)$ is part of a triangle in $G_{n^{\prime}}$ and has infinitely many neighbors, so it must be $a^{\prime}$. The arguments given for $D_{n}$ show that $f\left(d_{\langle\rangle}\right)$must be $d_{s}^{\prime}$ for some $s$, and $n^{\prime} \geq n$. Since $a$ and $d_{\langle\rangle}$are joined in $G_{n}$ by a path of length $n+2, a^{\prime}$ and $d_{s}^{\prime}$ must be joined in $G_{n^{\prime}}$ by a path of length at most $n+2$. This is impossible if $n^{\prime}>n$, so we must have $n^{\prime}=n$; therefore, the graphs $G_{n}$ have the required properties.

2.3. Linear orders and embeddings. As in the preceding subsection, we will take a simple $\xi_{\rho}$ monotone set and generalize it to obtain a wide variety of monotone degrees; in particular, we will be able to embed arbitrary countable (or larger if more is assumed) partial orderings into the $f_{\rho}$ monotone degrees. The example we will start with comes from the following question: must every $\mathcal{F}_{\rho}$ monotone degree strictly greater than the flat degree be as great as the degree given by Figure 2.2(b)? The answer is no, as is shown by Figure 3.4.

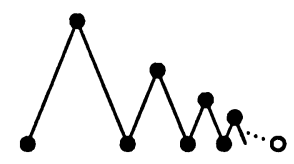

FIGURE 3.4. Another $\mathcal{F}_{\rho}$ monotone set

Note that the upper points in Figure 3.4 are arranged in an order of type $\omega$ (from left to right). We now generalize this by allowing the upper points to be arranged in an arbitrary (countably infinite) order type.

DEFINITION. Suppose that $\preceq$ is a linear ordering of $\omega$. A set $S \subseteq \omega$ is a cut (more specifically, a $\preceq$-cut) iff it is closed downward under $\preceq$. Define a monotone set $D(\preceq)$ by requiring it to have the following frontier (and using Proposition 2.1): a point $x \in \omega_{2}$ is in the frontier iff the sets $\{n \in \omega:(x(2 n), x(2 n+1))=(1,0)\}$ and $\{n \in \omega:(x(2 n), x(2 n+1)) \neq(0,1)\}$ are cuts which either are equal or differ by a single natural number $n$ such that $(x(2 n), x(2 n+1))=(1,1)$; the only frontier point of $D(\preceq)$ which is not in $D(\preceq)$ is $\overline{10}$.

The set represented by Figure 3.4 is $D(\leq)$. In general, the frontier of $D(\preceq)$ consists of 'lower' and 'upper' points. The lower points are incomparable under $\leq$, there is one for each $\preceq$-cut, and the topology on the lower points matches the order topology of the cuts under $\subseteq$ (which linearly orders the collection of $\preceq$-cuts). When two cuts are adjacent, instead of identifying them as in the Dedekind cut construction of the real numbers, we add an upper point which lies above the two corresponding lower points and above no other relevant point; there is one upper point for each member of the field of the original ordering $\preceq$. Let $e(c)$ denote the point in ${ }^{\omega_{2}}$ associated with the cut $c$, and let $e(n)$ for $n \in \omega$ denote the upper point connecting $e(\{m: m \prec n\})$ and $e(\{m: m \preceq n\})$.

Every nonempty collection of $\preceq$-cuts has a supremum (namely its union) and an infimum (its intersection). Using this, we show that $\operatorname{Fr}(D(\preceq))$ is m-connected (see 
section II.3), as follows: Suppose $\operatorname{Fr}(D(\preceq))$ is partitioned into two nonempty closed sets, $S$ and $T$, where $S$ is the one which contains $e(\varnothing)$. If $T$ contains no lower points, then it contains a point $e(n)$ lying above a point in $S$ (namely $e(\{m: m \prec n\}))$. Otherwise, let $c_{0}$ be the infimum of those cuts $c$ such that $e(c) \in T$. Since the topology on the points $e(c)$ corresponds to the order topology on the cuts $c$, and $T$ is closed, $e\left(c_{0}\right) \in T$. Now let $c_{1}$ be the supremum of all cuts $c \subset c_{0}$; since $S$ is closed, $e\left(c_{1}\right) \in S$, so $c_{1} \neq c_{0}$. Clearly there is no cut between $c_{1}$ and $c_{0}$, so $c_{0}=c_{1} \cup\{n\}$ for some $n \in \omega$; then $e(n)$ is in either $S$ or $T$ and lies above a point in $S$ and a point in $T$. Therefore, in any case, $S$ and $T$ are not m-disjoint.

On the other hand, if we delete any single point other than $e(\varnothing)$ or $e(\omega)$ from $\operatorname{Fr}(D(\preceq))$, the resulting set will not be m-connected. If the deleted point is $e(c)$ for some cut $c$, the remainder can be partitioned into $\left\{e\left(c^{\prime}\right): c^{\prime} \subset c\right\} \cup\{e(n): n \in c\}$ and $\left\{e\left(c^{\prime}\right): c^{\prime} \supset c\right\} \cup\{e(n): n \in \omega-c\}$; if it is $e(n)$ for $n \in \omega$, the remainder can be partitioned into $\{e(c): n \notin c\} \cup\{e(m): m \prec n\}$ and $\{e(c): n \in c\} \cup\{e(m): m \succ n\}$. In the latter case, the two m-connected components are closed in ${ }^{\omega_{2}}$.

At this point, it will be useful to introduce an interval notation. If $c \subseteq c^{\prime}$ are cuts, let $\left[e(c), e\left(c^{\prime}\right)\right]$ denote the set consisting of all $e\left(c^{\prime \prime}\right)$ where $c \subseteq c^{\prime \prime} \subseteq c^{\prime}$ and all $e(m)$ for $m \in c^{\prime}-c$. This set is clearly closed in $\operatorname{Fr}(D(\preceq))$ and hence in ${ }^{\omega_{2}}$. The argument above shows that $\left[e(c), e\left(c^{\prime}\right)\right]$ is m-connected. A point $x$ is in $\left[e(c), e\left(c^{\prime}\right)\right]$ if and only if $x=e(c), x=e\left(c^{\prime}\right)$, or $e(c)$ and $e\left(c^{\prime}\right)$ are in different m-connected components of $\operatorname{Fr}(D(\preceq))-\{x\}$. Notations for open and half-open intervals and intervals with an endpoint of the form $e(m)$ for $m \in \omega$ can be defined similarly.

We now consider the question of when $D(\preceq) \leq_{\mathrm{m}} D\left(\preceq^{\prime}\right)$, for linear orders $\preceq, \preceq^{\prime}$ of $\omega$. (Let $e^{\prime}(c)$ and $e^{\prime}(n)$ denote the analogues of $e(c)$ and $e(n)$ for $\preceq^{\prime}$.) Look at a related question first: when is there an m-continuous map $f: \operatorname{Fr}(D(\preceq)) \rightarrow \operatorname{Fr}\left(D\left(\preceq^{\prime}\right)\right)$ such that $f(\overline{01})=\overline{01}$ and $f(\overline{\mathbf{1 0}})=\overline{\mathbf{1 0}}$ ?

Suppose $f$ is such a map. Fix $n \in \omega$. The subset $S=\left[e^{\prime}\left(\left\{m: m \preceq^{\prime} n\right\}\right), e^{\prime}(\omega)\right]$ of $\operatorname{Fr}\left(D\left(\preceq^{\prime}\right)\right)$ is closed in $\omega_{2}$, and there is at least one $\preceq$-cut $c$ (namely $\omega$ ) such that $f(e(c)) \in S$, so there is a least such $\preceq$-cut; call it $c_{0}$. The set $\operatorname{Fr}\left(D\left(\preceq^{\prime}\right)\right)-S$ is also closed, so there is a greatest $\preceq$-cut $c \subseteq c_{0}$ such that $f(e(c)) \notin S$; call it $c_{1}$. Then $c_{1}$ and $c_{0}$ must be adjacent cuts, so $c_{0}=c_{1} \cup\{m\}$ for some $m \notin c_{1}$; call this $m h(n)$. Clearly no point in $S$ lies above a point in $\operatorname{Fr}\left(D\left(\preceq^{\prime}\right)\right)-S$, and the only point of $\operatorname{Fr}\left(D\left(\preceq^{\prime}\right)\right)-S$ lying above a point of $S$ is $e^{\prime}(n)$; since $e\left(c_{1}\right) \leq e(m) \geq e\left(c_{0}\right)$ and $f$ is relatively monotone, we must have $f(e(m))=e^{\prime}(n)$ and $f\left(e\left(c_{0}\right)\right)=e^{\prime}\left(\left\{k: k \preceq{ }^{\prime} n\right\}\right)$.

We have now defined a function $h: \omega \rightarrow \omega$ so that $\{k: k \preceq h(n)\}$ is the least $\preceq$-cut $c$ such that $f(e(c))=e^{\prime}\left(c^{\prime}\right)$ for a $\preceq^{\prime}$-cut $c^{\prime}$ containing $n$. If $n \preceq^{\prime} n^{\prime}$, then any $\preceq^{\prime}$-cut containing $n^{\prime}$ also contains $n$, so $\{k: k \preceq h(n)\} \subseteq\left\{k: k \preceq h\left(\overline{n^{\prime}}\right)\right\}$, so $h(n) \preceq \bar{h}\left(n^{\prime}\right)$. Since $f(e(h(n)))=e^{\prime}(n), h$ is one-to-one. Therefore, $h$ is an embedding of $\left(\omega, \preceq^{\prime}\right)$ into $(\omega, \preceq)$.

Conversely, suppose $h: \omega \rightarrow \omega$ is an embedding of $\left(\omega, \preceq^{\prime}\right)$ into $(\omega, \preceq)$. Then we can define $f: \operatorname{Fr}(D(\preceq)) \rightarrow \operatorname{Fr}\left(D\left(\preceq^{\prime}\right)\right)$ as follows: for any $\preceq$-cut $c, f(e(c))=$ $e^{\prime}\left(h^{-1}\right.$ " $\left.c\right)$; if $m=h(n)$, then $f(e(m))=e^{\prime}(n)$; if $m \in \omega$ is not in the range of $h$, then $f(e(m))=e^{\prime}(\{n: h(n) \prec m\})$. Since $\overline{01}=e(\varnothing)=e^{\prime}(\varnothing)$ and $\overline{10}=e(\omega)=e^{\prime}(\omega)$, we have $f(\overline{01})=\overline{01}$ and $f(\overline{10})=\overline{10}$. It is easy to verify that the definition of $f$ is equivalent to the formula $(f(x)(2 n), f(x)(2 n+1))=(x(2 h(n)), x(2 h(n)+1))$, so $f$ is $\mathrm{m}$-continuous. Therefore, a function $f$ with these properties exists iff $\left(\omega, \preceq^{\prime}\right)$ can be embedded into $(\omega, \preceq)$. 
Now, $D(\preceq) \leq_{\mathrm{m}} D\left(\preceq^{\prime}\right)$ is equivalent to the existence of a function $f: \operatorname{Fr}(D(\preceq)) \rightarrow$ $\operatorname{Fr}\left(D\left(\preceq^{\prime}\right)\right)$ with the same properties except " $f(\overline{01})=\overline{01}$," which is replaced with " $x \neq \overline{10}$ implies $f(x) \neq \overline{10}$." Suppose we have such an $f$, and fix $n_{0} \in \omega$ such that either $f(\overline{01})=e^{\prime}\left(n_{0}\right)$ or $f(\overline{01})=e^{\prime}(c)$ for some $\preceq^{\prime}$-cut $c$ not containing $n_{0}$. The definition of $h(n)$ above could break down because there is no $\preceq$-cut $c \subseteq c_{0}$ such that $f(c) \notin S$; however, this will not happen if $n_{0} \preceq^{\prime} n$. Therefore, $h$ is defined at least on a $\preceq^{\prime}$-final segment of $\omega$. Now, for any $m \in \omega,[e(\varnothing), e(\{i: i \prec m\})]$ is a compact subset of $\operatorname{Fr}(D(\preceq))$ not containing $\overline{10}$, so its image under $f$ is a compact set not containing $\overline{10}$; therefore, there is $k \in \omega$ such that no point in this image starts with $(10)^{k}$. We may assume $k>n_{0}$. Let $n$ be the $\preceq^{\prime}$-maximum of $\{i: i<k\}$; then there is no $\preceq$-cut $c$ not containing $m$ such that $f(e(c))=e^{\prime}\left(c^{\prime}\right)$ for a $\preceq^{\prime}$-cut $c^{\prime}$ containing $n$. Since $f(e(\{i: i \preceq h(n)\}))$ is a $\preceq^{\prime}$-cut containing $n$, we must have $m \in\{i: i \preceq h(n)\}$, so $m \preceq h(n)$. But $m$ was arbitrary, so $h$ must map $\left\{n: n_{0} \preceq^{\prime} n\right\}$ $\preceq$-cofinally into $\omega$.

Conversely, if the function $h$ embeds $\left(\left\{n: n_{0} \preceq^{\prime} n\right\}, \preceq^{\prime}\right)$ cofinally into $(\omega, \preceq)$, then we can define a function $f: \operatorname{Fr}(D(\preceq)) \rightarrow{ }^{\omega} 2$ by letting $(f(x)(2 n), f(x)(2 n+1))$ be $(x(2 h(n)), x(2 h(n)+1))$ if $n_{0} \preceq^{\prime} n,(1,0)$ otherwise. Clearly $f$ is m-continuous, and it is easy to see that $f(\overline{10})=\overline{10}$ and $f(x) \in \operatorname{Fr}\left(D\left(\preceq^{\prime}\right)\right)$ for all $x$. If $x$ is in the domain of $f$ but is not $\overline{10}$, then $(x(2 m), x(2 m+1)) \neq(1,0)$ for some $m$, which implies $\left(x\left(2 m^{\prime}\right), x\left(2 m^{\prime}+1\right)\right) \neq(1,0)$ for all $m^{\prime} \succeq m$; since the range of $h$ is $\preceq$-cofinal in $\omega,(x(2 h(n)), x(2 h(n)+1)) \neq(1,0)$ for some $n$, so $f(x) \neq \overline{10}$. This completes the proof of:

PROPOSITION 3.6. For any linear orderings $\preceq$ and $\preceq^{\prime}$ of $\omega, D(\preceq) \leq_{\mathrm{m}} D\left(\preceq^{\prime}\right)$ iff some final segment of $\left(\omega, \preceq^{\prime}\right)$ can be cofinally embedded into $(\omega, \preceq)$.

In particular, $D(\preceq) \equiv_{\mathrm{m}} D\left(\preceq^{\prime}\right)$ if $(\omega, \preceq)$ and $\left(\omega, \preceq^{\prime}\right)$ have the same order type, so we may write $D(\theta)$ for an order type $\theta$ to define a monotone degree.

Since $\overline{D(\preceq)}-D(\preceq)$ contains only one point, it is closed, so $D(\preceq)$ is $\mathcal{F}_{\rho}$. If $(\omega, \preceq)$ has a last element $m$, then $\operatorname{Fr}(D(\preceq)) \cap D(\preceq)=\operatorname{Fr}(D(\preceq)) \cap\left\{x \in{ }^{\omega} 2: x(2 m+1)=1\right\}$ is closed, so $D(\preceq)$ is also $\mathcal{F}_{\rho_{-}}$; since $e(m)>\overline{10}, D(\preceq)$ is monotone equivalent to the set in Figure 2.2(b). If $(\omega, \preceq)$ has no last element, then for each $k$ there is a cut $c \neq \omega$ containing all $i<k$; therefore, $\overline{10}$ is the limit of points $e(c)$ with $c \neq \omega$, so $D(\preceq)$ is strictly $\mathcal{F}_{\rho}$.

For any nonzero $\alpha, \beta<\omega_{1}$, an order of type $\omega^{\alpha}$ can be cofinally embedded into one of type $\omega^{\beta}$ if and only if $\alpha \leq \beta$. (If $\alpha \leq \beta$, map $\omega^{\alpha}$ to $\omega^{\beta}$ by sending $\gamma$ to $\omega^{\delta} \cdot \gamma$, where $\alpha+\delta=\beta$.) Hence, $D\left(\omega^{\alpha}\right)$ for $1 \leq \alpha<\omega_{1}$ is a decreasing $\omega_{1}$-sequence of monotone degrees. There are many other degrees of the form $D(\theta)$; some examples are $D\left(\omega^{*} \cdot \omega\right), D(\varsigma \cdot \omega), D\left(\varsigma^{\alpha} \cdot \omega^{\beta}\right)$, and $D(\eta)$, where $\varsigma$ and $\eta$ are the order types of the integers and the rationais, respectively. Since $\omega$ is cofinally embeddable into any countable linear order without last element, while any such order is cofinally embeddable into the rationals, $D(\omega)$ and $D(\eta)$ are the greatest and least monotone degrees of sets of the form $D(\preceq)$ for linear orders $(\omega, \preceq)$ without last element.

The collection of monotone degrees of sets of the form $D(\preceq)$ is not as large as it might appear from the above; in particular, it does not include an infinite antichain or ascending sequence. This follows from a result which is still called Fraïssé's Conjecture, although it is now a theorem of Laver: there is no infinite antichain or descending chain of countable order types under embeddability. (See 
Rosenstein [13, Chapter 10] for this result and the other terminology and results on linear orderings we will be using; the original proof of Fraïssé's Conjecture appears in Laver [8]. The proof uses more of the axiom of choice than we are assuming, but the result as stated above follows from our assumptions by absoluteness: if there were a counterexample, it could be coded by a sequence $s$ of natural numbers, and then there would be a counterexample in the inner model $L[s]$, which satisfies the full axiom of choice.) What we need here is the corresponding result for cofinal embeddability, which can be stated as follows: if $L_{n}$ for $n \in \omega$ are countable linear orders, then there are $n<n^{\prime}$ in $\omega$ such that $L_{n}$ can be cofinally embedded into $L_{n}^{\prime}$. We may assume that no $L_{n}$ has a last element, and hence each $L_{n}$ is isomorphic to a sum $\sum_{m \in \omega} L_{n m}$ for some linear orderings $L_{n m}$. Laver's results are strong enough to show that, for any countable linear orderings $L_{n m}$, there exist $n<n^{\prime}$ in $\omega$ and a strictly increasing function $h: \omega \rightarrow \omega$ such that $L_{n m}$ is embeddable into $L_{n^{\prime} h(m)}$ for each $m$; this immediately implies that $\sum_{m \in \omega} L_{n m}$ is cofinally embeddable into $\sum_{m \in \omega} L_{n^{\prime} m}$.

So we must come up with a new idea if we want to construct extremely many monotone degrees. A step in the right direction is as follows: Suppose we have a linear ordering $\preceq$ of $\omega$ with no last element. As noted above, $(\omega, \preceq)$ will be isomorphic to an $\omega$-sum of linear orderings. Equivalently, we can partition $\omega$ into nonempty sets $S_{n}$ for $n \in \omega$ so that $n<n^{\prime}$ implies $S_{n} \prec S_{n^{\prime}}$ (i.e., for any $m \in S_{n}$ and $\left.m^{\prime} \in S_{n^{\prime}}, m \prec m^{\prime}\right)$. We now define a monotone set $A$. The frontier of $A$ consists of all points of the form $i j x$ where $x \in \operatorname{Fr}(D(\preceq))$ and $i j$ is:

10 if $x \in\left(e\left(\bigcup_{k<n} S_{n}\right), e\left(\bigcup_{k \leq n} S_{n}\right)\right)$ for some even $n \in \omega$;

01 if $x \in\left(e\left(\bigcup_{k<n} S_{n}\right), e\left(\bigcup_{k \leq n} S_{n}\right)\right)$ for some odd $n \in \omega$;

01,11 , or 10 if $x=\overline{10}$ or $x=e\left(\bigcup_{k<n} S_{n}\right)$ for some $n \in \omega$.

The only frontier points of $A$ which are not in $A$ are $01 \overline{10}, 11 \overline{10}$, and $10 \overline{10}$. This set is depicted in Figure 3.5, where squiggle number $n$ represents

$$
\left[e\left(\bigcup_{k<n} S_{n}\right), e\left(\bigcup_{k \leq n} S_{n}\right)\right]
$$

prefixed with 10 (if $n$ is even) or 01 (if $n$ is odd).

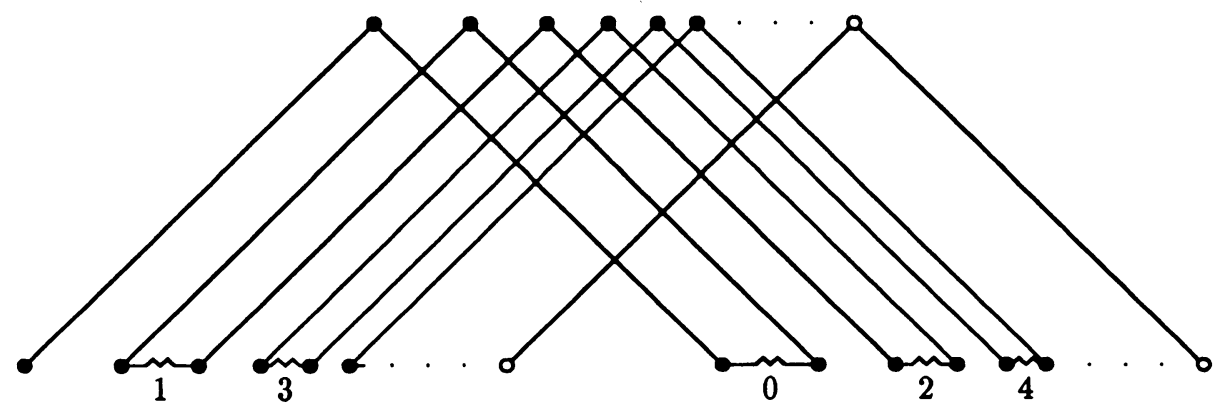

FIGURE 3.5. A variant of $D(\preceq)$ 
The frontier of $A$, like that of $D(\preceq)$, is m-connected. (The m-connected component of $\operatorname{Fr}(A)$ which contains $01 e(\varnothing)$ must also contain $11 e(\varnothing)$ and $10 e(\varnothing)$; then it must contain all of $10\left[e(\varnothing), e\left(S_{0}\right)\right]$ since this set is m-connected; then it contains $11 e\left(S_{0}\right)$ and $01 e\left(S_{0}\right)$, and then all of the points in $01\left[e\left(S_{0}\right), e\left(S_{0} \cup S_{1}\right)\right]$, and so

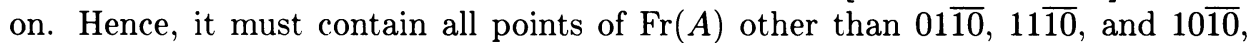
and since it is closed it must contain these three points as well.) However, if any point of $\operatorname{Fr}(A) \cap A$ other than $01 \overline{01}$ is deleted from $\operatorname{Fr}(A)$, the resulting set has two m-connected components, only one of which meets $\operatorname{Fr}(A)-A$.

Now, suppose $A^{\prime}$ is constructed in the same way from $\preceq^{\prime}$ and $\left\langle S_{n}^{\prime}: n \in \omega\right\rangle$; when will we have $A \leq_{\mathrm{m}} A^{\prime}$ ? A sufficient condition for this is that, for each $n$, $\left(S_{n}^{\prime}, \preceq^{\prime}\right)$ is embeddable into $\left(S_{n}, \preceq\right)$. Suppose this is so; then, by the argument preceding Proposition 3.6, for each $n$ there is an m-continuous function $f_{n}$ from $\left[e\left(\bigcup_{k<n} S_{k}\right), e\left(\bigcup_{k \leq n} S_{k}\right)\right]$ to $\left[e^{\prime}\left(\bigcup_{k<n} S_{k}^{\prime}\right), e^{\prime}\left(\bigcup_{k<n} S_{k}^{\prime}\right)\right]$ such that $f_{n}\left(e\left(\bigcup_{k<n} S_{k}\right)\right)$ $=e^{\prime}\left(\bigcup_{k<n} S_{k}^{\prime}\right)$ and $f_{n}\left(e\left(\bigcup_{k \leq n} S_{k}\right)\right)=e^{\prime}\left(\bigcup_{k \leq n} S_{k}^{\prime}\right)$. Now construct a map $f: \operatorname{Fr}(A)$ $\rightarrow \operatorname{Fr}\left(A^{\prime}\right)$ by specifying $f(i j \overline{10})=i j \overline{10}$ and $f(i j x)=i j f_{n}(x)$ for $x \in\left[e\left(\bigcup_{k<n} S_{k}\right)\right.$, $\left.e\left(\bigcup_{k \leq n} S_{k}\right)\right]$; it is easy to see that $f$ satisfies the hypotheses of Proposition 2.2 .

If this sufficient condition were also necessary, then we would immediately get at least $2^{\aleph_{0}}$ monotone degrees of this type the way we did with bushes at the end of the preceding subsection. The sufficient condition is not necessary, however; there are several ways in which it can be weakened. First, we can get by with having $\left(S_{n}^{\prime}, \preceq^{\prime}\right)$ embeddable into $\left(S_{n}, \preceq\right)$ only for $n \geq n_{0}$ for some fixed $n_{0}$; we then define $f(i j x)$ to be $i_{0} j_{0} e^{\prime}\left(\bigcup_{k<n_{0}} S_{k}^{\prime}\right)$ for all $i j x$ such that $x \in\left[e(\varnothing), e\left(\bigcup_{k<n} S_{k}\right)\right)$, where $i_{0} j_{0}$ is 01 if $n_{0}$ is even and 10 if $n_{0}$ is odd, and otherwise proceed as before. Second, it will suffice to have $\left(S_{n+m}^{\prime}, \preceq^{\prime}\right)$ embeddable into $\left(S_{n}, \preceq\right)$ for all sufficiently large $n$, where $m$ is a fixed integer (positive or negative); this is again much as before, except that now $f$ will send $i j e\left(\bigcup_{k<n} S_{k}\right)$ to $i j e^{\prime}\left(\bigcup_{k<n+m} S_{k}^{\prime}\right)$ or $j i e^{\prime}\left(\bigcup_{k<n+m} S_{k}^{\prime}\right)$ for sufficiently large $n$, depending on whether $m$ is even or odd. A third weaker sufficient condition is that $A \leq_{\mathrm{m}} A^{\prime}$ if there is a strictly increasing function $h: \omega \rightarrow \omega$ such that $h(n) \equiv$ $n(\bmod 2)$ and $\left(S_{n}^{\prime}, \preceq^{\prime}\right)$ is embeddable into $\left(S_{h(n)}, \preceq\right)$ for all $n$; again we proceed as in the basic case, but now $f$ sends ije $\left(\bigcup_{k<n} S_{k}\right)$ to $i j e^{\prime}\left(\bigcup_{k<m} S_{k}^{\prime}\right)$, where $m$ is least such that $h(m) \geq n$, and if $x \in\left[e\left(\bigcup_{k<n} S_{k}\right), e\left(\bigcup_{k \leq n} S_{k}\right)\right]$ where $n$ is not in the range of $h$, then $f(i j x)=i j e^{\prime}\left(\bigcup_{k<m} S_{k}^{\prime}\right)$ with $m$ as above.

The first two of these weakenings do not hinder us in constructing a large collection of monotone degrees; in fact, we will see that the second one is actually helpful. The third one, however, is fatal; using it and Laver's results, we can show that the monotone degrees constructed in this way do not include an infinite antichain or ascending chain. We now modify the construction so as to eliminate the third weakening.

Suppose, as before, that $\preceq$ is a linear ordering of $\omega$ and $\left\{S_{n}: n \in \omega\right\}$ is a partition of $\omega$ into nonempty sets so that $m \in S_{n}, m^{\prime} \in S_{n^{\prime}}$, and $n<n^{\prime}$ imply $m \prec m^{\prime}$. Assume that no $S_{n}$ has a first or last element under $\preceq$. (This assumption causes no problems, since instead of using given order types $\theta_{n}$ for $S_{n}$ we can use order types $\varsigma+\theta_{n}+\varsigma$ or $\varsigma \cdot \theta_{n}$; this will work just as well since $\varsigma+\theta+\varsigma$ is embeddable into $\varsigma+\theta^{\prime}+\varsigma$ iff $\theta$ is embeddable into $\theta^{\prime}$, and similarly for $\varsigma \cdot \theta$.) Define a monotone set $D_{2}\left(\preceq,\left\langle S_{n}: n \in \omega\right\rangle\right)$ by turning the even-numbered 'arches' in Figure 3.5 upsidedown, as in Figure 3.6. 


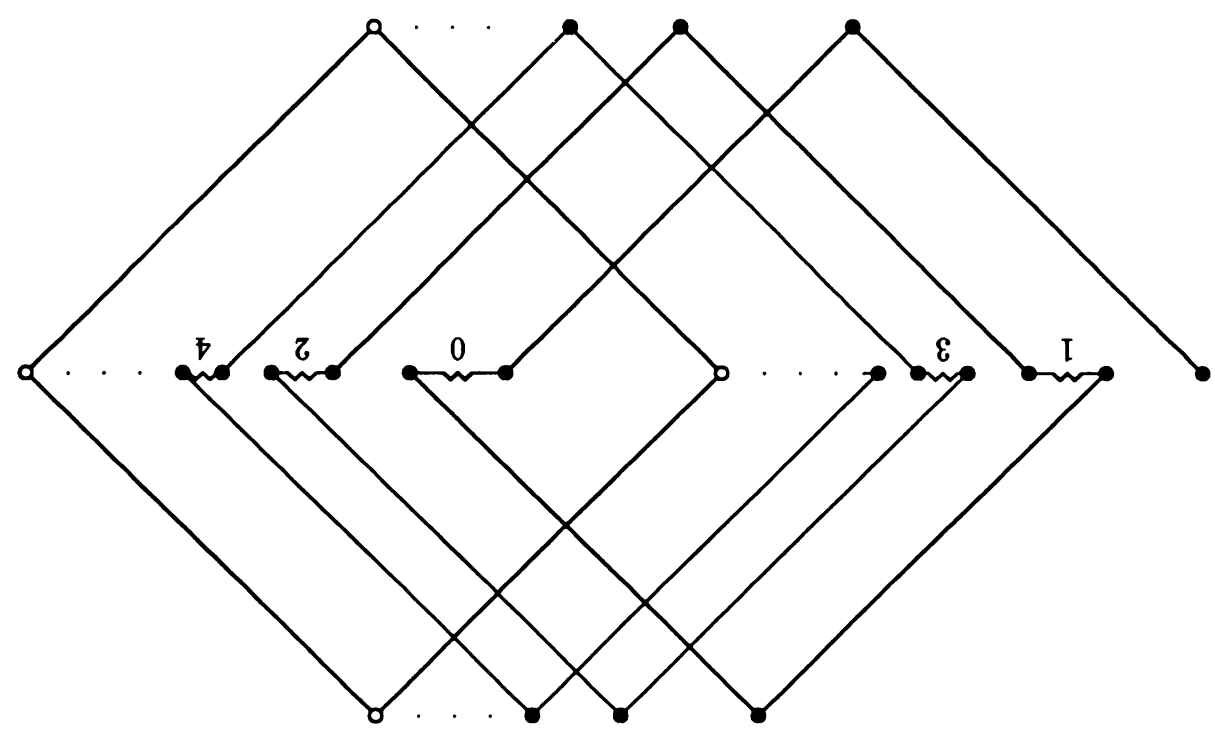

FigURE 3.6. The set $D_{2}\left(\preceq,\left\langle S_{n}: n \in \omega\right\rangle\right)$

More formally, we define this set by requiring its frontier to consist of all points of the form $i j x$ where $x \in \operatorname{Fr}(D(\preceq))$ and $i j$ is:

$$
\begin{aligned}
& 10 \text { if } x \in\left(e\left(\bigcup_{k<n} S_{n}\right), e\left(\bigcup_{k \leq n} S_{n}\right)\right) \text { for some even } n \in \omega ; \\
& 01 \text { if } x \in\left(e\left(\bigcup_{k<n} S_{n}\right), e\left(\bigcup_{k \leq n} S_{n}\right)\right) \text { for some odd } n \in \omega ;
\end{aligned}
$$

01,00 , or 10 if $x=e\left(\bigcup_{k<n} S_{n}\right)$ for some even $n \in \omega$;

01,11 , or 10 if $x=e\left(\bigcup_{k<n} S_{n}\right)$ for some odd $n \in \omega$;

$01,00,10$, or 11 if $x=\overline{10}$.

The only frontier points which are not in the set are $01 \overline{10}, 00 \overline{10}, 10 \overline{10}$, and $11 \overline{10}$. (If we had not assumed that $S_{n}$ has no first or last element, then we would have to add a few extra points to this frontier to make it $\mathrm{Mon}_{\rho}$; this would add a few extra cases to the following arguments but probably would not cause any real difficulties.)

As in the case of the set in Figure 3.5 (and by the same proof), the frontier of $D_{2}\left(\preceq,\left\langle S_{n}: n \in \omega\right\rangle\right)$ is m-connected, but removing any point of it other than $01 e(\varnothing)$ or $i j \overline{10}$ leaves a set with two m-connected components, one of which is entirely contained in $D_{2}\left(\preceq,\left\langle S_{n}: n \in \omega\right\rangle\right)$.

To see that we have met the objection that arose from the preceding idea, we must determine when one set of this type is monotone reducible to another.

PROPOSITION 3.7. Suppose $\preceq$ and $\preceq$ are linear orderings of $\omega$, and $\left\{S_{n}: n \in\right.$ $\omega\}$ and $\left\{S_{n}^{\prime}: n \in \omega\right\}$ are partitions of $\omega$ into nonempty sets $S_{n}$ and $S_{n}^{\prime}$ such that, for each $n, i \in S_{n}$ and $j \in S_{n+1}$ imply $i \prec j$, and similarly for $S_{n}^{\prime}$. Assume that $S_{n}\left(S_{n}^{\prime}\right)$ has no first or last element under $\preceq\left(\preceq^{\prime}\right)$. Then $D_{2}\left(\preceq,\left\langle S_{n}: n \in \omega\right\rangle\right) \bigsqcup_{m}$ $D_{2}\left(\preceq^{\prime},\left\langle S_{n}^{\prime}: n \in \omega\right\rangle\right)$ iff there is an even integer $m$ (positive or negative) such that, for all sufficiently large $n,\left(S_{n+m}^{\prime}, \preceq^{\prime}\right)$ is embeddable into $\left(S_{n}, \preceq\right)$. 
ProOF. First we introduce some abbreviations. We will write $D_{2}$ instead of $D_{2}\left(\preceq,\left\langle S_{n}: n \in \omega\right\rangle\right)$, and $D_{2}^{\prime}$ instead of $D_{2}\left(\preceq^{\prime},\left\langle S_{n}^{\prime}: n \in \omega\right\rangle\right)$. Let $S_{<n}=\bigcup_{k<n} S_{k}$, and $S_{\leq n}=S_{<n+1}$; similarly for $S_{<n}^{\prime}$ and $S_{<n}^{\prime}$.

If $m$ is even and $\left(S_{n+m}^{\prime}, \preceq^{\prime}\right)$ is embeddable into $\left(S_{n}, \preceq\right)$ for all $n \geq n_{0}$, then for each $n \geq n_{0}$ there is an m-continuous function $f_{n}$ from $\left[e\left(S_{<n}\right), e\left(S_{\leq n}\right)\right]$ to $\left[e^{\prime}\left(S_{<n+m}^{\prime}\right), e^{\prime}\left(S_{\leq n+m}^{\prime}\right)\right]$ such that $f_{n}\left(e\left(S_{<n}\right)\right)=e^{\prime}\left(S_{<n+m}^{\prime}\right)$ and $f_{n}\left(e\left(S_{\leq n}\right)\right)=$ $e^{\prime}\left(S_{\leq n+m}^{\prime}\right)$. (This follows from the argument preceding Proposition 3.6.) We may assume that $n_{0}$ is even. Define a map $f: \operatorname{Fr}\left(D_{2}\right) \rightarrow \operatorname{Fr}\left(D_{2}^{\prime}\right)$ by: for any $i, j \in\{0,1\}$,

$$
f(i j x)= \begin{cases}01 e^{\prime}\left(S_{<n_{0}+m}^{\prime}\right) & \text { if } x \in\left[e(\varnothing), e\left(S_{<n_{0}}\right)\right), \\ i j f_{n}(x) & \text { if } n \geq n_{0} \text { and } x \in\left[e\left(S_{<n}\right), e\left(S_{\leq n}\right)\right], \\ i j \overline{10} & \text { if } x=\overline{10} .\end{cases}
$$

It is straightforward to verify that $f$ satisfies the hypotheses of Proposition 2.2, so $D_{2} \leq_{\mathrm{m}} D_{2}^{\prime}$.

Conversely, suppose $f: \operatorname{Fr}\left(D_{2}\right) \rightarrow \operatorname{Fr}\left(D_{2}^{\prime}\right)$ is m-continuous, and $f(x)$ is of the form $i j \overline{10}$ for some $i, j \in\{0,1\}$ iff $x$ is of this form. Then $f(01 e(\varnothing))=i_{0} j_{0} x_{0}$ for some $i_{0}, j_{0} \in\{0,1\}$ and some $x_{0} \in D\left(\preceq^{\prime}\right)$ other than $\overline{10}$; fix $N \in \omega$ such that $x_{0} \in\left[e^{\prime}(\varnothing), e^{\prime}\left(S_{<N}^{\prime}\right)\right)$. For any point of $\operatorname{Fr}\left(D_{2}^{\prime}\right)$ of the form $i j e^{\prime}\left(S_{<n}^{\prime}\right)$ with $n>N, f(01 e(\varnothing))$ and $f(01 \overline{10})$ are in different m-connected components of $\operatorname{Fr}\left(D_{2}^{\prime}\right)-$ $\left\{i j e^{\prime}\left(S_{<n}^{\prime}\right)\right\}$; since $f$ is m-continuous and its domain is m-connected, $i j e^{\prime}\left(S_{<n}^{\prime}\right)$ must be in the range of $f$. Since $i j \overline{10}=\lim _{k \rightarrow \infty} i j e^{\prime}\left(S_{<k}^{\prime}\right)$, compactness implies that the four points $i j \overline{10}$ are all in the range of $f$. There are only two ways in which this can happen: either $f(i j \overline{10})=i j \overline{10}$ for all $i, j$, or $f(i j \overline{10})=j i \overline{10}$ for all $i, j$.

Assume that the first of these two cases holds. Since $f$ is continuous, there is $n_{0} \in \omega$ such that, for all $n \geq n_{0}, f(i j x) \in i j^{\omega} 2$ for any $i, j \in\{0,1\}$ and $x \in\left[e\left(S_{<n}\right), e\left(S_{\leq n}\right)\right]$. In particular, $f\left(i i e\left(S_{<n_{0}}\right)\right)$ must be $i i e^{\prime}\left(S_{<n_{0}+m}^{\prime}\right)$ for some even integer $m$, where $i$ is 0 if $n_{0}$ is even, 1 otherwise.

We now prove by induction on $n \geq n_{0}$ that $f\left(i i e\left(S_{<n}\right)\right)=i i e^{\prime}\left(S_{<n+m}^{\prime}\right)$ where $i$ is 0 if $n$ is even and 1 otherwise, $f\left(j i e\left(S_{<n}\right)\right)=j i e^{\prime}\left(S_{<n+m}^{\prime}\right)$ where $j=1-i, f(x) \in$ $j i\left[e^{\prime}\left(S_{<n+m}^{\prime}\right), e^{\prime}\left(S_{\leq n+m}^{\prime}\right)\right]$ for all $x \in j i\left[e\left(S_{<n}\right), e\left(S_{\leq n}\right)\right]$, and $f\left(j i e\left(S_{\leq n}\right)\right)=$ $j i e^{\prime}\left(S_{<n+m}^{\prime}\right)$. Suppose that $f\left(i i e\left(S_{<n}\right)\right)=i i e^{\prime}\left(S_{<n+m}^{\prime}\right)$. Then the only point of $\operatorname{Fr}\left(D_{2}^{\prime}\right) \cap j i^{\omega} 2$ above or below $f\left(i i e\left(S_{<n}\right)\right)$ is jie $\left(S_{<n+m}^{\prime}\right)$, so $f\left(j i e\left(S_{<n}\right)\right)$ $=j i e^{\prime}\left(S_{<n+m}^{\prime}\right)$. The two sets $E=j i\left[e^{\prime}\left(S_{<n+m}^{\prime}\right), e^{\prime}\left(S_{\leq n+m}^{\prime}\right)\right]$ and $\operatorname{Fr}\left(D_{2}^{\prime}\right) \cap$ $j i^{\omega_{2}}-E$ are m-disjoint closed subsets of ${ }^{\omega} 2$; since $f$ maps the m-connected set $j i\left[e\left(S_{<n}\right), e\left(S_{\leq n}\right)\right]$ into the union of these two sets, it must map this interval into one of the two sets. Therefore, $f(x) \in E$ for all $x \in j i\left[e\left(S_{<n}\right), e\left(S_{\leq n}\right)\right]$. The only points $y \leq z$ or $y \geq z$ such that $y \in E$ and $z \in \operatorname{Fr}\left(D_{2}^{\prime}\right) \cap j j^{\omega} 2$ are $y=j i e^{\prime}\left(S_{\leq n+m}^{\prime}\right)$ and $z=j j e^{\prime}\left(S_{\leq n+m}^{\prime}\right)$, so $f\left(j i e\left(S_{\leq n}\right)\right)=j i e^{\prime}\left(S_{\leq n+m}^{\prime}\right)$ and $f\left(j j e\left(S_{<n+1}\right)\right)=j j e^{\prime}\left(S_{<n+1+m}^{\prime}\right)$. This completes the induction.

In particular, for $n \geq n_{0}$, the map

$$
f_{n}:\left[e\left(S_{<n}\right), e\left(S_{\leq n}\right)\right] \rightarrow\left[e^{\prime}\left(S_{<n+m}^{\prime}\right), e^{\prime}\left(S_{\leq n+m}^{\prime}\right)\right]
$$

defined by $f(j i x)=j i f_{n}(x)$ is m-continuous, and it sends $e\left(S_{<n}\right)$ to $e^{\prime}\left(S_{<n+m}^{\prime}\right)$ and $e\left(S_{\leq n}\right)$ to $e^{\prime}\left(S_{\leq n+m}^{\prime}\right)$; therefore, by the argument preceding Proposition 3.6, $\left(S_{n+m}^{\prime}, \preceq^{\prime}\right)$ is embeddable into $\left(S_{n}, \preceq\right)$ for all $n \geq n_{0}$.

This completes the first case. Now suppose the second case holds; by following the same argument, we can show that there exist $n_{0} \in \omega$ and $m \geq n_{0}$ such that, 
for all $n \geq n_{0}, f\left(i i e\left(S_{<n}\right)\right)=i i e^{\prime}\left(S_{<m-n}^{\prime}\right)$. This clearly gives a contradiction for $n>m$. Therefore, the second case cannot arise, so we are done.

In particular, the monotone degree of $D_{2}\left(\preceq,\left\langle S_{n}: n \in \omega\right\rangle\right)$ depends only on the order types of $\left(S_{n}, \preceq\right)$ for $n \in \omega$, so it makes sense to write $D_{2}\left(\left\langle\theta_{n}: n \in \omega\right\rangle\right)$ for countable (nonempty) order types $\theta_{n}$ to define a monotone degree.

Applying $D_{2}$ to the many order types we have already considered (e.g., the $\left(\omega_{1}+1\right)$-chain consisting of $\zeta \cdot \omega^{\alpha}$ for $\alpha<\omega_{1}$ and $\left.\eta\right)$ gives an extremely large collection of $\mathcal{F}_{\rho}$ monotone degrees; in fact, the operation $D_{2}$ is almost too general. We therefore restrict it to a more tractable case. First, let

$$
D_{2}^{0}\left(\left\langle\theta_{n}: n \in \omega\right\rangle\right)=D_{2}\left(\left\langle\theta_{0}, \zeta \cdot \omega^{*}, \theta_{1}, \zeta \cdot \omega^{*}, \zeta \cdot \omega, \theta_{2}, \varsigma \cdot \omega^{*}, \zeta \cdot \omega, \varsigma \cdot \omega, \theta_{3}, \ldots\right\rangle\right),
$$

where there are one $\zeta \cdot \omega^{*}$ and $n \zeta \cdot \omega$ 's between $\theta_{n}$ and $\theta_{n+1}$. Suppose

$$
D_{2}^{0}\left(\left\langle\theta_{n}: n \in \omega\right\rangle\right) \leq_{\mathrm{m}} D_{2}^{0}\left(\left\langle\theta_{n}^{\prime}: n \in \omega\right\rangle\right) .
$$

Let $m$ be the shift value obtained when Proposition 3.7 is applied to these two sets. If $m$ were nonzero, then there would be arbitrarily large $n$ such that the $n$th order type in the list to which $D_{2}$ is applied for the first set is $\varsigma \cdot \omega^{*}$ while the $(n+m)$ th order type in the other list is $\zeta \cdot \omega$, or vice versa; since neither of $\zeta \cdot \omega$ and $\zeta \cdot \omega^{*}$ is embeddable into the other, this is impossible. Therefore, $m$ must be zero. This proves that $D_{2}^{0}\left(\left\langle\theta_{n}: n \in \omega\right\rangle\right) \leq_{\mathrm{m}} D_{2}^{0}\left(\left\langle\theta_{n}^{\prime}: n \in \omega\right\rangle\right)$ iff $\theta_{n}^{\prime}$ is embeddable into $\theta_{n}$ for all sufficiently large $n$.

Next, fix a bijective pairing function $j: \omega \times \omega \rightarrow \omega$, let $\pi_{1}(n)$ be the first coordinate of $j^{-1}(n)$, and define $D_{2}^{1}\left(\left\langle\theta_{n}: n \in \omega\right\rangle\right)$ to be $D_{2}^{0}\left(\left\langle\theta_{\pi_{1}(n)}: n \in \omega\right\rangle\right)$; then

$$
D_{2}^{1}\left(\left\langle\theta_{n}: n \in \omega\right\rangle\right) \leq_{\mathrm{m}} D_{2}^{1}\left(\left\langle\theta_{n}^{\prime}: n \in \omega\right\rangle\right)
$$

iff $\theta_{n}^{\prime}$ is embeddable into $\theta_{n}$ for all $n$ (not just for sufficiently large $n$ ). Then applying $D_{2}^{1}$ to just the simple order types $\zeta \cdot 2$ and $\zeta$ gives an embedding of $(\mathcal{P}(\omega), \subseteq)$ into the $\mathcal{F}_{\rho}$ monotone degrees; as we saw in the preceding subsection, this gives us chains and antichains of size $2^{\aleph_{0}}$. However, we can do even better by using $D_{2}^{0}$ instead of $D_{2}^{1}$.

Here, too, we can look at just the order types $\zeta \cdot 2$ and $\zeta$. For any $z \subseteq \omega$, define $D_{2}^{0}(z)$ to be $D_{2}^{0}\left(\left\langle\theta_{n}: n \in \omega\right\rangle\right)$, where $\theta_{n}$ is $\zeta$ if $n \in z$ and $\varsigma \cdot 2$ if $n \notin z$. Then $D_{2}^{0}(z) \leq_{\mathrm{m}} D_{2}^{0}\left(z^{\prime}\right)$ iff $z \subseteq^{*} z^{\prime}$, where $z \subseteq^{*} z^{\prime}$ means that $z-z^{\prime}$ is finite. In particular, $D_{2}^{0}(z) \equiv_{\mathrm{m}} D_{2}^{0}\left(z^{\prime}\right)$ iff $z \equiv^{*} z^{\prime}$ (i.e., $z \subseteq^{*} z^{\prime} \subseteq^{*} z$ ). If we use the more common notation $\mathcal{P}(\omega) /$ finite instead of $\mathcal{P}(\omega) / \equiv^{*}$, then we have:

THEOREM 3.8. The partial ordering $\left(\mathcal{P}(\omega) /\right.$ finite, $\left.\subseteq^{*}\right)$ can be embedded into the $\mathcal{F}_{\rho}$ monotone degrees under $\leq_{\mathrm{m}}$.

It is well known that the partial ordering $\left(P(\omega) /\right.$ finite, $\left.\subseteq^{*}\right)$ is very powerful. For instance, Keisler [5] shows, assuming the axiom of choice, that every Boolean algebra of size at most $\aleph_{1}$ can be embedded into the Boolean algebra $P(\omega) /$ finite, and the same proof shows without choice that every countable Boolean algebra can be embedded into $P(\omega)$ /finite. It is easy to show that any partial ordering can be embedded into the underlying partial ordering of a Boolean algebra (since we can embed $(S, \leq)$ into $(\mathcal{P}(S), \subseteq)$ by sending $a \in S$ to $\{b \in S: b \leq a\})$; by taking a subalgebra, we may assume that the algebra is generated by the range of the embedding, and hence has size at most $\aleph_{\alpha}$ if the partial order has size at most $\aleph_{\alpha}$. 
Therefore, any countable partial order (or any partial order of size at most $\aleph_{1}$ if choice is assumed) can be embedded into $\left(P(\omega) /\right.$ finite, $\left.\subseteq^{*}\right)$. Kunen [6, ex. II $\left.(23)\right]$ shows that ZFC together with Martin's Axiom implies that any Boolean algebra (and hence any partial order) of size less than $2^{\aleph_{0}}$ can be embedded into $P(\omega)$ /finite. Laver [7] constructs a model of ZFC in which every linear order of size at most $2^{\aleph_{0}}$ can be embedded into $\left({ }^{\omega} \omega, \prec\right)$, where $f \prec g$ means $\lim _{n \rightarrow \infty} g(n)-f(n)=+\infty$, while $2^{\aleph_{0}}$ is large; it is not difficult to modify the forcing notion to get a model of ZFC with $2^{\aleph_{0}}$ large in which every Boolean algebra of size at most $2^{\aleph_{0}}$ can be embedded into $P(\omega)$ /finite. (In fact, it is slightly simpler to handle $P(\omega) /$ finite rather than $\left({ }^{\omega} \omega, \prec\right)$, since the numbers $m_{\beta, p}$ are no longer required; Laver's result follows from this because $\left({ }^{\omega} 2,<^{*}\right)$, which is isomorphic to $\left(P(\omega), \subset^{*}\right)$, can be embedded into $\left({ }^{\omega} \omega, \prec\right)$ by the map $F$ defined by $F(a)(n)=\sum_{i=0}^{n} 2^{i} a(i)$.)

To summarize: any partial order of 'reasonable' size can be embedded into $\left(P(\omega) /\right.$ finite,$\left.\subseteq^{*}\right)$ and hence into the $\mathcal{F}_{\rho}$ monotone degrees under $\leq_{\mathrm{m}}$.

2.4. Semilattice embeddings. We have gotten very general results about embeddings of partial orders into the monotone degrees. However, if we want to preserve least upper bounds (that is, if we want to embed an upper semilattice into the upper semilattice of monotone degrees under $\leq_{\mathrm{m}}$ and $\mathrm{jn}^{+}$), then the results of the preceding subsection are not suitable, because all of the sets constructed there had $\mathrm{m}$-connected frontiers and were therefore in $\mathrm{jn}^{+}$-prime monotone degrees. In this subsection we will see that we can get general results for semilattices as well, at least in the countable case.

THEOREM 3.9. Any countable upper semilattice can be embedded into the $\mathcal{F}_{\rho}$ monotone degrees under $\leq_{\mathrm{m}}$ and $\mathrm{jn}^{+}$.

PrOOF. First, we note that any countable upper semilattice can be embedded into the upper semilattice underlying a countable atomic Boolean algebra. To see this, let $\left(X, \leq_{X}, \vee_{X}\right)$ be the upper semilattice, and map $X$ to $P(X)$ by letting $f(x)=\left\{y \in X: x \mathbb{Z}_{X} y\right\}$ for $x \in X$; then take the subalgebra of $\mathcal{P}(X)$ generated by $\{\{x\}: x \in X\} \cup f$ " $X$.

So suppose we have a countable atomic Boolean algebra $B$, with elements $\left\{b_{n}: n \in\right.$ $\omega\}$, operations $\vee, \wedge$, and - , associated partial order $\leq$, greatest element 1 , and least element 0 . Using the results of the preceding subsection, we can associate with each atom $a$ of $B$ a monotone strictly $\mathcal{F}_{\rho}$ set $A(a)$ with m-connected frontier such that $A(a) \mathbb{Z}_{\mathrm{m}} A\left(a^{\prime}\right)$ if $a \neq a^{\prime}$ and $A(a)$ is not in the 'flat' $\mathcal{F}_{\rho}$ degree (i.e., $A(a)_{b}<_{\mathrm{m}} A(a)$ ).

Define a sequence $\left\langle W_{n}: n \in \omega\right\rangle$ of subsets of $B$ as follows: $W_{0}=\{1\}$, and, for each $n, W_{n+1}=\left(\left\{c \wedge b_{n}: c \in W_{n}\right\} \cup\left\{c-b_{n}: c \in W_{n}\right\}\right)-\{0\}$. Then each set $W_{n}$ is a finite maximal antichain, each element of $W_{n}$ is the sum of one or two elements of $W_{n+1}$, and every element of $B$ is the sum of a subset of $W_{n}$ for all sufficiently large $n$. In particular, every atom of $B$ is in $W_{n}$ for all sufficiently large $n$.

For each $c \in \bigcup_{k \in \omega} W_{k}$ and each $n \in \omega$, define a set $B_{n}(c)$ as follows: if $c \notin$ $\bigcup_{k \leq n} W_{k}$, let $B_{n}(c)=\varnothing$; if $c \in W_{n}$, let $B_{n}(c)$ be $A(c)$ if $c$ is an atom and $\varnothing$ otherwise; if $c \in W_{k}$ but $c \notin W_{k+1}$ for some $k<n$, let

$$
B_{n}(c)=\mathrm{jn}^{+}\left(B_{n}\left(c \wedge b_{k}\right), B_{n}\left(c-b_{k}\right)\right) .
$$

It is easy to verify that $B_{n}(c) \subseteq B_{n+1}(c)$ (using a backward induction on the greatest $k \leq n+1$ such that $\left.c \in W_{k}\right)$. Now let $B(c)=\bigcup_{n \in \omega} B_{n}(c)$. 
If $c \in W_{k}$ but $c \notin W_{k+1}$, then the 'continuity' of $\mathrm{jn}^{+}$implies that $B(c)=$ $\mathrm{jn}^{+}\left(B\left(c \wedge b_{k}\right), B\left(c-b_{k}\right)\right)$. Clearly $B(a)=A(a)$ for any atom $a$.

Suppose $x \in \operatorname{Fr}(B(c)) \cap B(c)$. Then $x$ is a limit of points which are not in $B(c)$ and hence not in $B_{n}(c)$ for any $n$; therefore, $x \in \operatorname{Fr}\left(B_{n}(c)\right) \cap B_{n}(c)$ for all sufficiently large $n$. Fix such an $n$; then $c$ must be in $W_{m}$ for some $m \leq n$. Now, by induction on $k$, we see that for each $k$ from $m$ to $n$ there is $c_{k} \in W_{k}$ and a finite sequence $s_{k} \in{ }^{<\omega} 2$ such that $c_{k} \leq c, s_{k} \subset x$, and $B_{n}(c) \cap s_{k}{ }^{\omega} 2=s_{k} B_{n}\left(c_{k}\right)$; furthermore, any frontier point of $B_{n}(c)$ which is not in $s_{k}{ }^{\omega_{2}}$ must be incomparable with all points in $s_{k}{ }^{\omega}$ 2. If we start with $n^{\prime} \geq n$ instead of $n$ and repeat this construction, we will get the same values of $c_{k}$ and $s_{k}$ for $k \leq n$; therefore, $B_{n^{\prime}}(c) \cap s_{n}{ }^{\omega} 2=s_{n} B_{n^{\prime}}\left(c_{n}\right)$ for all $n^{\prime} \geq n$, so $B(c) \cap s_{n}{ }^{\omega_{2}}=s_{n} B\left(c_{n}\right)$. Another induction on $k \leq n$ shows that any frontier point of $B(c)$ is either in $s_{k}{ }^{\omega} 2$ or incomparable with all points of $s_{k}{ }^{{ }{ }^{2}} 2$. Since $s_{n} \subset x$ and $B_{n}(c) \cap s_{n}{ }^{\omega_{2}}=s_{n} B_{n}\left(c_{n}\right), B_{n}\left(c_{n}\right)$ is nonempty, so $c_{n}$ must be an atom. Therefore, we have shown that for each $c \in \bigcup_{n \in \omega} W_{n}$ and each $x \in \operatorname{Fr}(B(c)) \cap B(c)$, there is $s \in{ }^{<\omega_{2}}$ and an atom $a \leq c$ such that $s \subset x, B(c) \cap s^{\omega} 2=s A(a)$, and any frontier point of $B(c)$ is either in $s^{\omega} 2$ or incomparable with all points of $s^{\omega} 2$.

Since $A(a)$ is $\mathcal{F}_{\rho}$, it follows immediately that no point $x \in \operatorname{Fr}(B(c)) \cap B(c)$ can be a limit of points in $\operatorname{Fr}(B(c))-B(c)$; therefore, $B(c)$ is $\mathcal{F}_{\rho}$.

Now, for any $b \in B$ which is not in $\bigcup_{n \in \omega} W_{n}$, define $B(b)$ as follows: let $N$ be least such that $b$ is a sum of elements of $W_{N}$, let $\left\langle c_{k}: k<m\right\rangle$ be a list of those $c \in W_{N}$ such that $c \leq b$, and let

$$
B(b)=\mathrm{jn}^{+}\left(B\left(c_{0}\right), \mathrm{jn}^{+}\left(B\left(c_{1}\right), \ldots, \mathrm{jn}^{+}\left(B\left(c_{k-2}\right), B\left(c_{k-1}\right)\right) \cdots\right)\right) .
$$

(Let $B(0)$ be $X_{b}$ for some $\xi_{\rho} X$.) The monotone degree of $B(b)$ is the least upper bound of the degrees of $B(c)$ for those $c \in W_{N}$ such that $c \leq b$ (if $b \neq 0$ ).

In fact, for any $n$ such that $b$ is a sum of elements of $W_{n}$, the degree of $B(b)$ is the least upper bound of the degrees of $B(c)$ for those $c \in W_{n}$ such that $c \leq b$ (if $b \neq 0)$. This is proved by induction on $n \geq N$; if it holds for $n$, then it holds for $n+1$ because each $c \in W_{n}$ either is in $W_{n+1}$ or is split into $c \wedge b_{n}, c-b_{n} \in W_{n+1}$ where $B(c)=\mathrm{jn}^{+}\left(B\left(c \wedge b_{n}\right), B\left(c-b_{n}\right)\right)$.

It follows immediately that $B\left(b \vee b^{\prime}\right)$ is a least upper bound of $B(b)$ and $B\left(b^{\prime}\right)$ (just take $n$ so large that both $b$ and $b^{\prime}$ are sums of elements of $W_{n}$ ). If $b \leq b^{\prime}$, then $b \vee b^{\prime}=b^{\prime}$, so $B(b) \leq_{\mathrm{m}} B\left(b^{\prime}\right)$. It remains to show that if $b \not b^{\prime}$, then $B(b) \underline{\mathrm{m}}_{\mathrm{m}} B\left(b^{\prime}\right)$. Since $b \leq b^{\prime}, b-b^{\prime} \neq 0$, so there is an atom $a \leq b-b^{\prime}$; since $A(a)=B(a) \leq_{\mathrm{m}} B(b)$, it will suffice to show $A(a) \mathbb{Z}_{\mathrm{m}} B\left(b^{\prime}\right)$. This is true by assumption if $b^{\prime}=0$, so assume $b^{\prime} \neq 0$. Suppose $A(a) \leq_{\mathrm{m}} B\left(b^{\prime}\right)$. Since $A(a)$ is $\mathrm{jn}^{+}$-prime, there exists $c^{\prime} \in \bigcup_{n \in \omega} W_{n}$ such that $c^{\prime} \leq b^{\prime}$ and $A(a) \leq_{\mathrm{m}} B\left(c^{\prime}\right)$. Suppose $f:{ }^{\omega_{2}} \rightarrow{ }^{\omega_{2}}$ witnesses $A(a) \leq_{\mathrm{m}} B\left(c^{\prime}\right)$. Fix $x \in \operatorname{Fr}(A(a)) \cap A(a)$; then $f(x) \in \operatorname{Fr}\left(B\left(c^{\prime}\right)\right) \cap B\left(c^{\prime}\right)$, so there is $s \in{ }^{<\omega_{2}} 2$ and an atom $a^{\prime} \leq c^{\prime}$ such that $s \subset x, B\left(c^{\prime}\right) \cap s^{\omega} 2=s A\left(a^{\prime}\right)$, and any frontier point of $B\left(c^{\prime}\right)$ is either in $s^{\omega} 2$ or incomparable with all points of $s^{\omega} 2$. Since $a^{\prime} \leq c^{\prime} \leq b^{\prime}, a^{\prime} \neq a$. Now, let $E=\operatorname{Fr}\left(B\left(c^{\prime}\right)\right) \cap s^{\omega} 2$; then $E$ and $\operatorname{Fr}\left(B\left(c^{\prime}\right)\right)-E$ are $\mathrm{m}$-disjoint closed sets. Since $f$ is m-continuous and maps $\operatorname{Fr}(A(a))$, which is $\mathrm{m}$ connected, into the union of these two sets, $f$ must map $\operatorname{Fr}(A(a))$ into one of the two sets; therefore, $f^{\text {" }} \operatorname{Fr}(A(a)) \subseteq E$. But $E=s \operatorname{Fr}\left(A\left(a^{\prime}\right)\right)$, so if $g: \operatorname{Fr}(A(a)) \rightarrow \operatorname{Fr}\left(A\left(a^{\prime}\right)\right)$ is defined by $f(x)=s g(x)$, then $g$ satisfies the hypotheses of Proposition 2.2, so $A(a) \leq_{\mathrm{m}} A\left(a^{\prime}\right)$, which is a contradiction. 
This method can be extended to give embeddings of certain uncountable semilattices into the $\mathcal{F}_{\rho}$ monotone degrees; in particular, the upper semilattice of cofinite subsets of $\omega_{2}$ can be embedded. A sketch of the proof is as follows: Note that the construction of $B(c)$ for $c \in \bigcup_{k \in \omega} W_{k}$ in the proof of the preceding theorem actually gave the minimal solution to the simultaneous equations

$$
B(c)= \begin{cases}A(c) & \text { if } c \text { is an atom, } \\ \mathrm{jn}^{+}\left(B\left(c \wedge b_{k}\right), B\left(c-b_{k}\right)\right) & \text { if } c \in W_{k}-W_{k+1} .\end{cases}
$$

Now choose $\mathcal{F}_{\rho}$ sets $A(s)$ for $s \in<\omega 2$ with m-connected frontiers such that $A(s) \not_{\mathrm{m}}$ $A\left(s^{\prime}\right)$ for $s \neq s^{\prime}$. For each $i \in\{0,1\}$ and each cofinite $G \subseteq \omega_{2}$, define the sets $B_{i}(G, s)$ for $s \in{ }^{<\omega} 2$ to be the minimal solution to the simultaneous equations

$$
B_{i}(G, s)=\mathrm{jn}^{+}\left(A_{i}(G, s), \mathrm{jn}^{+}\left(B_{i}(G, s 0), B_{i}(G, s 1)\right)\right),
$$

where

$$
\left(A_{0}(G, s), A_{1}(G, s)\right)= \begin{cases}(\varnothing, A(s)) & \text { if } s^{\omega} 2 \subseteq G, \\ (A(s), \varnothing) & \text { otherwise. }\end{cases}
$$

Let $C(G)=\mathrm{jn}^{+}\left(B_{0}(G,\langle\rangle), B_{1}(G,\langle\rangle)\right)$; then $G \mapsto C(G)$ is the desired embedding. To see this, we must show that $C(G) \leq_{\mathrm{m}} C\left(G^{\prime}\right)$ iff $G \subseteq G^{\prime}$. If $G \nsubseteq G^{\prime}$, fix $x \in G-G^{\prime}$, find $n \in \omega$ such that $\left(x\lceil n)^{\omega} 2 \subseteq G\right.$, and let

$$
A^{\prime}=\mathrm{jn}_{\omega}^{+}(\langle A((x\lceil n) 0), A((x\lceil n), 1), A((x\lceil(n+1)) 0), A((x\lceil(n+1)) 1), \ldots\rangle),
$$

where $\mathrm{jn}_{\omega}^{+}$is defined in the next subsection; then $A^{\prime} \leq_{\mathrm{m}} B_{1}(G, x \mid n) \leq_{\mathrm{m}} C(G)$ but $A^{\prime} \not_{\mathrm{m}} C\left(G^{\prime}\right)$, so $C(G) \mathbb{Z}_{\mathrm{m}} C\left(G^{\prime}\right)$. On the other hand, if $G \subseteq G^{\prime}$, find $n \in \omega$ such that, for every $s \in{ }^{n} 2$, either $G^{\prime} \cap s^{\omega} 2=G \cap s^{\omega} 2$ or $s^{\omega} 2 \subseteq G^{\prime}$. In the former case, $B_{i}(G, s)=B_{i}\left(G^{\prime}, s\right) \leq_{\mathrm{m}} C\left(G^{\prime}\right)$; in the latter, one can apply Proposition 2.2 to the identity map on $\operatorname{Fr}\left(B_{i}(G, s)\right)$ to get $B_{i}(G, s) \leq_{\mathrm{m}} B_{1}\left(G^{\prime}, s\right) \leq_{\mathrm{m}} C\left(G^{\prime}\right)$. Also, $A(s) \leq_{\mathrm{m}} C\left(G^{\prime}\right)$ for all $s \in{ }^{<\omega} 2$. Since $C(G)$ is obtained from these sets by applying $\mathrm{jn}^{+}$finitely many times, $C(G) \leq_{\mathrm{m}} C\left(G^{\prime}\right)$, as desired.

On the other hand:

PROPOSITION 3.10. (a) There is a semilattice of size $\aleph_{1}$ which cannot be embedded into the monotone degrees.

(b) No uncountable subalgebra of the Boolean algebra $\mathcal{P}(\omega)$ containing all singletons can be embedded as an upper semilattice into the monotone degrees.

PROOF. (a) Define an upper semilattice as follows: the elements are $a, b_{\alpha}$ for $\alpha<\omega_{1}$, and $c_{\alpha}$ for $\alpha<\omega_{1} ; b_{\alpha}<a$ for all $\alpha ; b_{\alpha} \not b_{\beta}$ if $\alpha \neq \beta ; c_{\alpha}<c_{\beta}$ for $\alpha<\beta$; and, for any $\alpha$ and $\beta, c_{\alpha} \leq b_{\beta}$ iff $\alpha \leq \beta$. Suppose $i$ is an embedding of this semilattice into the monotone degrees, and fix $A \in i(a)$. For each $\beta$, if $B \in i\left(b_{\beta}\right)$ and $C \in i\left(c_{\beta+1}\right)$, then $A \equiv_{\mathrm{m}} \mathrm{jn}^{+}(B, C)$, so by the proof of Proposition 2.3 there are clopen sets $X_{\beta}$ and $Y_{\beta}$ such that, if $B^{\prime}=A-X_{\beta}$ and $C^{\prime}=A-Y_{\beta}$, then $B^{\prime} \leq_{\mathrm{m}} B$, $C^{\prime} \leq_{\mathrm{m}} C$, and $A \equiv_{\mathrm{m}} \mathrm{jn}^{+}\left(B^{\prime}, C^{\prime}\right)$. Since $a \not b_{\beta}$ and $c_{\alpha+1} \leq b_{\beta}$ for $\alpha<\beta, C^{\prime} \not C^{\prime \prime}$ for $C^{\prime \prime} \in i\left(c_{\alpha+1}\right)$ if $\alpha<\beta$. Therefore, $Y_{\alpha} \neq Y_{\beta}$ for $\alpha<\beta$, which is impossible since there are only countably many clopen subsets of ${ }^{\omega} 2$.

(b) Suppose that $i$ is such an embedding, and fix $A \in i(\omega)$. For each $n \in \omega$, let $F_{n}$ be the set of all $x \in \operatorname{Fr}(A)$ such that, whenever $B \in i(\omega-\{n\})$ and $f$ witnesses $A \leq_{\mathrm{m}} \mathrm{jn}^{+}(B, A), f(x)$ must be in $10^{\omega} 2$. Then $F_{n}$ is the intersection of $f^{-1}$ " $10^{\omega} 2$ for all such $f$, so $F_{n}$ is closed. Furthermore, this intersection cannot be 
empty. Suppose it is; then, by compactness, some finite subintersection is empty, so there is a finite sequence $\left\langle f_{k}: k<N\right\rangle$ of $f$ 's as above such that for any $x \in \operatorname{Fr}(A)$ there is $k<N$ such that $f_{k}(x) \in 01^{\omega} 2$. The obvious way of constructing a map witnessing $\mathrm{jn}^{+}\left(B_{0}, A\right) \leq_{\mathrm{m}} \mathrm{jn}^{+}\left(B_{1}, A\right)$ from a map witnessing $B_{0} \leq_{\mathrm{m}} B_{1}$ gives a map which sends $01^{\omega} 2$ into $01^{\omega} 2$; therefore, we may assume that all of the $B$ 's corresponding to the functions $f_{k}$ are the same. Now define $g: \operatorname{Fr}(A) \rightarrow \operatorname{Fr}(B)$ by $01 g(x)=f_{k}(x)$, where $k$ is least such that $f_{k}(x) \in 01^{\omega} 2$; this $g$ satisfies the hypotheses of Proposition 2.2, so $A \leq_{\mathrm{m}} B$, contradicting $i(\omega-\{n\})<_{\mathrm{m}} i(\omega)$.

If $W \subseteq \omega$ is such that $\overline{\bigcup_{n \in W} F_{n}}$ and $\overline{\bigcup_{n \in \omega-W} F_{n}}$ are disjoint, then by $\operatorname{Sep}_{\mathrm{I}}(\mathcal{F})$ these two sets must be separable by a clopen set, and this clopen set clearly determines $W$ since the sets $F_{n}$ are nonempty. Since the subalgebra was assumed to be uncountable, it must contain a set $W$ such that there is a point $y \in \overline{\bigcup_{n \in W} F_{n}} \cap \overline{\bigcup_{n \in \omega-W} F_{n}}$. Fix $B \in i(W)$ and $C \in i(\omega-W)$, and let $g$ be a witness to $A \leq_{\mathrm{m}} \mathrm{jn}^{+}(B, C)$. If $g(y) \in 01^{\omega} 2$, choose $n \in \omega-W$ and $x \in F_{n}$ so close to $y$ that $g(x) \in 01^{\omega} 2$; if $g(y) \in 10^{\omega} 2$, choose $n \in W$ and $x \in F_{n}$ such that $g(x) \in 10^{\omega} 2$. We have $B \leq_{\mathrm{m}} B_{n}$ in the former case and $C \leq_{\mathrm{m}} B_{n}$ in the latter, where $B_{n} \in i(\omega-\{n\})$, and $B, C \leq_{\mathrm{m}} A$ in either case; hence, we can easily construct $f$ witnessing $A \leq_{\mathrm{m}} \mathrm{jn}^{+}\left(B_{n}, A\right)$ such that $f(x) \in 01^{\omega_{2}}$. This contradicts $x \in F_{n}$, so we are done.

This proposition shows that one cannot modify the construction above for cofinite subsets of $\omega_{2}$ to get an embedding of the upper semilattice of open subsets of $\omega_{2}$ into the monotone degrees: if $\left\langle x_{n}: n \in \omega\right\rangle$ is a sequence of distinct points in ${ }^{\omega_{2}}$ converging to $x$, then the lattice of sets $G \subseteq{ }^{\omega_{2}}$ such that $\{x\} \subseteq{ }^{\omega_{2}}-G \subseteq$ $\{x\} \cup\left\{x_{n}: n \in \omega\right\}$ is isomorphic to $P(\omega)$, and all such sets are open.

2.5. Greatest lower bounds and $\omega$-ary least upper bounds. The results in this subsection are not as general as those in previous subsections; they consist mainly of examples of degrees which have or do not have greatest lower bounds or least upper bounds. Some examples can be obtained trivially by looking at corresponding examples for Wadge degrees; for example, a strictly $\Sigma_{2}^{0}$ set and a strictly $\Pi_{2}^{0}$ set cannot have a greatest lower bound, and a collection consisting of one strictly $\mathrm{Ha}_{n}$ set for each $n \in \omega$ cannot have a least upper bound. Here, however, we will look only at strictly $\mathcal{F}_{\rho}$ monotone sets.

The sets constructed in subsection 2.3 are of such a wide variety that it makes sense to look among them for examples of pairs of degrees with and without greatest lower bounds. In fact, one might expect the degrees $D(\preceq)$ alone to provide such examples; surprisingly, this is not the case.

PROPOSITION 3.11. If $\theta_{n}^{0}$ and $\theta_{n}^{1}$ are countable (nonzero) order types such that $\theta_{n}^{0}$ is embeddable into $\theta_{n+1}^{0}$ and $\theta_{n}^{1}$ is embeddable into $\theta_{n+1}^{1}$ for each $n$, then $D\left(\sum_{n \in \omega}\left(\theta_{n}^{0}+\theta_{n}^{1}\right)\right)$ is a greatest lower bound of $D\left(\sum_{n \in \omega} \theta_{n}^{0}\right)$ and $D\left(\sum_{n \in \omega} \theta_{n}^{1}\right)$.

ProOF. Fix linear orderings $\preceq^{0}, \preceq^{1}$, and $\preceq$ of $\omega$ and partitions $\left\{S_{n}^{0}: n \in \omega\right\}$, $\left\{S_{n}^{1}: n \in \omega\right\}$, and $\left\{S_{n}: n \in \omega\right\}$ of $\omega$ such that $S_{n}^{0} \prec^{0} S_{n+1}^{0}$ (i.e., $i \prec^{0} j$ for any $i \in S_{n}^{0}$ and $\left.j \in S_{n+1}^{0}\right), S_{n}^{1} \prec^{1} S_{n+1}^{1}, S_{n} \prec S_{n+1},\left(S_{n}^{0}, \preceq^{0}\right)$ and $\left(S_{2 n}, \preceq\right)$ have order type $\theta_{n}^{0}$, and $\left(S_{n}^{1}, \preceq^{1}\right)$ and $\left(S_{2 n+1}, \preceq\right)$ have order type $\theta_{n}^{1}$. Let $A$ be any monotone subset of ${ }^{\omega_{2}} 2$, and suppose that $f^{i}:{ }^{\omega_{2}} \rightarrow{ }^{\omega_{2}}$ witnesses $A \leq_{\mathrm{m}} D\left(\preceq^{i}\right)$ for $i=0,1$. We will construct a map $f: \operatorname{Fr}(A) \rightarrow \operatorname{Fr}(D(\preceq))$ satisfying the hypotheses of Proposition 2.2. 
First, we define numbers $m_{n}^{0}, m_{n}^{1} \in \omega$ for $n \in \omega$. Let $m_{0}^{0}=0$. Now suppose $m_{n}^{0}$ is given. Let $E=\operatorname{Fr}(A) \cap\left(f^{0}\right)^{-1}$ " $\left[e^{0}(\varnothing), e^{0}\left(S_{<m_{n}^{0}+1}^{0}\right)\right]$ (see subsection 2.3, including the beginning of the proof of Proposition 3.7, for the notation used here). Then $E$ is a compact subset of $\operatorname{Fr}(A) \cap A$, so $f^{1}$ " $E$ is a compact subset of $\operatorname{Fr}\left(D\left(\preceq^{1}\right)\right) \cap D\left(\preceq^{1}\right)$; therefore, $f^{1}$ " $E \subseteq\left[e^{1}(\varnothing), e^{1}\left(S_{<m-1}^{1}\right)\right]$ for some $m>0$. Let $m_{n}^{1}$ be the least such $m$ which is greater than $m_{n-1}^{1}+1$ (if $n>0$ ). Similarly, given $m_{n}^{1}$, let $E=$ $\operatorname{Fr}(A) \cap\left(f^{1}\right)^{-1}$ " $\left[e^{1}(\varnothing), e^{1}\left(S_{<m_{n}^{1}+1}^{1}\right)\right]$, and let $m_{n+1}^{0}$ be the least $m>m_{n}^{0}+1$ such that $f^{0}$ " $E \subseteq\left[e^{0}(\varnothing), e^{0}\left(S_{<m-1}^{0}\right)\right]$.

By hypothesis, $\theta_{n}^{0}$ is embeddable into $\theta_{m_{n}^{0}}^{0}$ for each $n$; hence, we can choose a continuous, relatively monotone function $g_{n}^{0}:\left[e^{0}\left(S_{<m_{n}^{0}}^{0}\right), e^{0}\left(S_{\leq m_{n}^{0}}^{0}\right)\right] \rightarrow\left[e\left(S_{<2 n}\right)\right.$, $\left.e\left(S_{\leq 2 n}\right)\right]$ such that $g_{n}^{0}\left(e^{0}\left(S_{<m_{n}^{0}}^{0}\right)\right)=e\left(S_{<2 n}\right)$ and $g_{n}^{0}\left(e^{0}\left(S_{\leq m_{n}^{0}}^{0}\right)\right)=e\left(S_{\leq 2 n}\right)$. Define maps $g_{n}^{1}:\left[e^{1}\left(S_{<m_{n}^{1}}^{1}\right), e^{1}\left(S_{\leq m_{n}^{1}}^{1}\right)\right] \rightarrow\left[e\left(S_{<2 n+1}\right), e\left(S_{\leq 2 n+1}\right)\right]$ similarly. Now define $f$ as follows: if $x=\overline{10}$, let $f(x)=\overline{10}$; if $f^{i}(x) \in\left[e^{i}\left(S_{<m_{n}^{i}}^{i}\right), e^{i}\left(S_{\leq m_{n}^{i}}^{i}\right)\right]$ for some $n \in \omega$ and $i<2$, let $f(x)=g_{n}^{i}\left(f^{i}(x)\right)$; if $f^{0}(x) \notin\left[e^{0}(\varnothing), e^{0}\left(S_{<m_{n}^{0}+1}^{0}\right)\right]$ and $f^{1}(x) \in\left[e^{1}(\varnothing), e^{1}\left(S_{<m_{n}^{1}}^{1}\right)\right]$ for some $n$, let $f(x)=e\left(S_{<2 n+1}\right)$; if $f^{1}(x) \notin\left[e^{1}(\varnothing)\right.$, $\left.e^{1}\left(S_{<m_{n}^{1}+1}^{1}\right)\right]$ and $f^{0}(x) \in\left[e^{0}(\varnothing), e^{0}\left(S_{<m_{n+1}^{1}}^{0}\right)\right]$ for some $n$, let $f(x)=e\left(S_{<2 n+2}\right)$. It is not hard to show that $f$ is well defined and has the required properties.

PROPOSITION 3.12. For any two countably infinite order types $\theta^{0}$ and $\theta^{1}$ without last element, $D\left(\theta^{0}\right)$ and $D\left(\theta^{1}\right)$ have a greatest lower bound, which is also of the form $D(\theta)$ for some $\theta$.

PROOF. An order type $\tau$ is called right indecomposable if, for any nonempty order types $\tau_{0}$ and $\tau_{1}$ such that $\tau=\tau_{0}+\tau_{1}, \tau$ is embeddable into $\tau_{1}$. It follows from Fraïsse's Conjecture that every countable order type $\tau$ has a final segment which is right indecomposable (since otherwise we could easily construct a sequence of final segments of $\tau$ which was a strictly decreasing sequence under embeddability). Since the monotone degree of $D(\tau)$ depends only on final segments of $\tau$, we may assume that $\theta^{0}$ and $\theta^{1}$ are right indecomposable.

Suppose $(\omega, \preceq)$ is right indecomposable and has no last element. Define inductively sets $S_{n} \subset \omega$ as follows: Given $S_{k}$ for $k<n$ such that $\bigcup_{k<n} S_{k}$ is a proper $\preceq$ initial segment of $\omega$, let $h_{n}: \omega \rightarrow \omega$ be an embedding of $(\omega, \preceq)$ into $\left(\omega-\bigcup_{k<n} S_{k}, \preceq\right)$. Choose $j \in \omega$ such that $j \notin \bigcup_{k<n} S_{k}$, and let $m$ be the maximum under $\preceq$ of $h_{n}(j)$ and $n$. Now let $S_{n}=\{i: i \preceq m\}-\bigcup_{k<n} S_{k}$. It is easy to see that the order type of $(\omega, \preceq)$ is the sum of the order types of $\left(S_{n}, \preceq\right)$ for $n \in \omega$, and $\left(S_{n}, \preceq\right)$ is embeddable into $\left(S_{n+1}, \preceq\right)$. Therefore, we can express $\theta^{i}$ as a sum $\sum_{n \in \omega} \theta_{n}^{2}$ for $i=0,1$ so that the hypotheses of Proposition 3.11 are met, so we are done.

Thus, the sets $D(\theta)$ do not provide us with an example of two monotone degrees without a greatest lower bound. Such examples do exist, however, among the sets $D_{2}\left(\left\langle\theta_{n}: n \in \omega\right\rangle\right)$.

PROPOSITION 3.13. Suppose $\theta_{n}$ and $\theta_{n}^{\prime}$ are countable (nonzero) order types without first or last element for $n \in \omega$, and neither of $\theta_{n}$ and $\theta_{m}^{\prime}$ is embeddable into the other for any $n$ and $m$. Then the sets $D_{2}\left(\left\langle\theta_{n}: n \in \omega\right\rangle\right)$ and $D_{2}\left(\left\langle\theta_{n}^{\prime}: n \in \omega\right\rangle\right)$ have no greatest lower bound under $\leq_{\mathrm{m}}$. 
PROOF. We again use the notation that was used in the proof of Proposition 3.7. Let $\preceq, \preceq^{\prime}, S_{n}$, and $S_{n}^{\prime}$ be as in Proposition 3.7 , so that $\left(S_{n}, \preceq\right)$ has order type $\theta_{n}$ and $\left(S_{n}^{\prime}, \preceq^{\prime}\right)$ has order type $\theta_{n}^{\prime}$.

Recall from $\S I I .1$ that we can treat ${ }^{\omega_{2}}$ and ${ }^{\omega_{2}} \times{ }^{\omega_{2}}$ interchangeably. Define functions $\pi$ and $\pi^{\prime}$ from ${ }^{\omega_{2}} 2 \times^{\omega_{2}} 2$ to ${ }^{\omega_{2}}$ by $\pi\left(x, x^{\prime}\right)=x$ and $\pi^{\prime}\left(x, x^{\prime}\right)=x^{\prime}$.

Suppose $A \subseteq \omega_{2}$ is a lower bound of $D_{2}$ and $D_{2}^{\prime}$; let $f$ and $f^{\prime}$ be witnesses to this. Define $F:{ }^{\omega_{2}} \rightarrow{ }^{\omega_{2}} 2 \times^{\omega_{2}}$ by $F(x)=\left(f(x), f^{\prime}(x)\right)$. We will construct a set which is also a lower bound of $D_{2}$ and $D_{2}^{\prime}$, but is not reducible to $A$; this will show that $A$ is not a greatest lower bound of $D_{2}$ and $D_{2}^{\prime}$. To do this, it will suffice to construct a closed set $E \subset \operatorname{Fr}\left(D_{2}\right) \times \operatorname{Fr}\left(D_{2}^{\prime}\right)$ such that: $E$ is a difference of monotone sets (so Proposition 2.1 can be applied to it); if $\left(x, x^{\prime}\right) \in E$, then $x \in D_{2}$ iff $x^{\prime} \in D_{2}^{\prime}$ (so the maps $\pi\left\lceil E\right.$ and $\pi^{\prime}\lceil E$ satisfy the hypotheses of Proposition 2.2); and there is no continuous, relatively monotone map $g$ from $E$ to $F$ " $F r(A)$ such that $\pi(x) \in D_{2}$ iff $\pi(g(x)) \in D_{2}$ (this implies that there is no continuous monotone $\tilde{g}:{ }^{\omega} 2 \times{ }^{\omega}{ }_{2} \rightarrow{ }^{\omega_{2}}$ reducing the monotone set constructed from $E$ to $A$, since otherwise we could put $g=F \circ \tilde{g})$.

Suppose we have a strictly increasing function $h: \omega \rightarrow \omega$ such that $h(n)$ is even for all $n$. Define a set $E \subset \operatorname{Fr}\left(D_{2}\right) \times \operatorname{Fr}\left(D_{2}^{\prime}\right)$ by: a point $\left(i j x, i^{\prime} j^{\prime} x^{\prime}\right) \in \operatorname{Fr}\left(D_{2}\right) \times$ $\operatorname{Fr}\left(D_{2}^{\prime}\right)$, where $i, j, i^{\prime}, j^{\prime} \in\{0,1\}$, is in $E$ iff either $x=x^{\prime}=\overline{10}$ or there is $n \in \omega$ such that either $i^{\prime} j^{\prime} x^{\prime}=01 e^{\prime}\left(S_{<2 n}^{\prime}\right)$ and $x \in\left[e\left(S_{<h(n)}\right), e\left(S_{<h(n+1)}\right)\right)$, or $i j x=01 e\left(S_{<h(n+1)}\right)$ and $x^{\prime} \in\left[e^{\prime}\left(S_{<2 n}^{\prime}\right), e^{\prime}\left(S_{<2(n+1)}^{\prime}\right)\right)$. It is straightforward to verify that $E$ meets the first two conditions mentioned above; we will show that the function $h$ can be chosen so that $E$ meets the third condition as well.

Suppose $g: E \rightarrow \operatorname{Fr}\left(D_{2}\right) \times \operatorname{Fr}\left(D_{2}^{\prime}\right)$ is continuous and, for all $x \in E, \pi(x) \in D_{2}$ iff $\pi(g(x)) \in D_{2}$ iff $\pi^{\prime}(g(x)) \in D_{2}^{\prime}$. Then, for any two-bit sequences $i j$ and $i^{\prime} j^{\prime}$, $g\left(i j \overline{10}, i^{\prime} j^{\prime} \overline{10}\right)$ must be $\left(\tilde{\imath} \tilde{\jmath} \overline{10}, \tilde{\imath}^{\prime} \tilde{j}^{\prime} \overline{10}\right)$ for some two-bit sequences $\tilde{\imath} \tilde{\jmath}$ and $\tilde{\imath}^{\prime} \tilde{j}^{\prime}$. Considering $\tilde{\imath} \tilde{j}$ for the case $i^{\prime} j^{\prime}=01$, look at two possibilities: either $\pi(g(i j \overline{10}, 01 \overline{10}))=$ $i j \overline{10}$ for all $i j$, or something else happens. Let us refer to the first of these possibilities by saying that " $g$ maps the first loop positively in the first coordinate." We make similar definitions for the second loop and/or the second coordinate: " $g$ maps the first loop positively in the second coordinate" means $\pi^{\prime}(g(i j \overline{10}, 01 \overline{10}))=$ $i j \overline{10}$ for all $i j$, " $g$ maps the second loop positively in the first coordinate" means $\pi(g(01 \overline{10}, i j \overline{10}))=i j \overline{10}$ for all $i j$, and " $g$ maps the second loop positively in the second coordinate" means $\pi^{\prime}(g(01 \overline{10}, i j \overline{10}))=i j \overline{10}$ for all $i j$.

Since $g$ is continuous, there is $n_{0} \in \omega$ such that, for any $\left(i j x, i^{\prime} j^{\prime} x^{\prime}\right) \in E$ such that $x^{\prime} \notin\left[e^{\prime}(\varnothing), e^{\prime}\left(S_{<2 n}^{\prime}\right)\right)$, we have

$$
\pi\left(g\left(i j x, i^{\prime} j^{\prime} x^{\prime}\right)\right) \uparrow 2=\pi\left(g\left(i j \overline{10}, i^{\prime} j^{\prime} \overline{10}\right)\right)\lceil 2
$$

and

$$
\pi^{\prime}\left(g\left(i j x, i^{\prime} j^{\prime} x^{\prime}\right)\right)\left\lceil 2=\pi^{\prime}\left(g\left(i j \overline{10}, i^{\prime} j^{\prime} \overline{10}\right)\right)\lceil 2 .\right.
$$

Now, using the arguments that were used in the proof of Proposition 3.7, one can prove the following facts: Suppose $n>n_{0}$. If $g$ maps the first loop positively in the first coordinate, and

$$
\pi\left(g\left(01 e\left(S_{<h(n)}\right), 01 e^{\prime}\left(S_{<2 n}^{\prime}\right)\right)\right) \in 01\left[e\left(S_{<h(n)+m}\right), e\left(S_{\leq h(n)+m}\right)\right]
$$

for some integer $m$, then

$$
\pi\left(g\left(01 e\left(S_{<h(n+1)}\right), 01 e^{\prime}\left(S_{<2 n}^{\prime}\right)\right)\right) \in 01\left[e\left(S_{<h(n+1)+m}\right), e\left(S_{\leq h(n+1)+m}\right)\right],
$$


and $\theta_{k+m}$ is embeddable into $\theta_{k}$ for all $k$ such that $h(n) \leq k<h(n+1)-1$. If $g$ does not map the first loop positively in the first coordinate, and

$$
\pi\left(g\left(01 e\left(S_{<h(n)}\right), 01 e^{\prime}\left(S_{<2 n}^{\prime}\right)\right)\right) \in i j\left[e(\varnothing), e\left(S_{<m}\right)\right) \cup\left\{01 e\left(S_{<m}\right)\right\}
$$

for some even natural number $m$ and some two-bit sequence $i j$, then

$$
\pi\left(g\left(01 e\left(S_{<h(n+1)}\right), 01 e^{\prime}\left(S_{<2 n}^{\prime}\right)\right)\right) \in i j\left[e(\varnothing), e\left(S_{<m}\right)\right) \cup\left\{01 e\left(S_{<m}\right)\right\}
$$

and, for all $x$ such that $\left(x, 01 e^{\prime}\left(S_{<2 n}^{\prime}\right)\right) \in E$,

$$
\pi\left(g\left(x, 01 e^{\prime}\left(S_{<2 n}^{\prime}\right)\right)\right) \in i^{\prime} j^{\prime}\left[e(\varnothing), e\left(S_{\leq m}\right)\right]
$$

for some two-bit $i^{\prime} j^{\prime}$. Similar statements hold for the second loop and/or the second coordinate.

In particular, if $g$ maps the first loop positively in the second coordinate, then $\theta_{k+m}^{\prime}$ is embeddable into $\theta_{k}$ for some $k$ and $m$; since we have assumed that this is not the case, $g$ cannot map the first loop positively in the second coordinate. Similarly, $g$ cannot map the second loop positively in the first coordinate. But clearly $g$ maps some loop positively in the first coordinate, since otherwise, fixing some $n>n_{0}$ and some even $m$ such that

$$
\pi\left(g\left(01 e\left(S_{<h(n)}\right), 01 e^{\prime}\left(S_{<2 n}^{\prime}\right)\right)\right) \in i j\left[e(\varnothing), e\left(S_{<m}\right)\right) \cup\left\{01 e\left(S_{<m}\right)\right\}
$$

for some $i j$, we would get

$$
\pi\left(g\left(01 e\left(S_{<h(N)}\right), 01 e^{\prime}\left(S_{<2 N}^{\prime}\right)\right)\right) \in i j\left[e(\varnothing), e\left(S_{<m}\right)\right) \cup\left\{01 e\left(S_{<m}\right)\right\}
$$

for all $N>n$, which is impossible because $\pi(g(01 \overline{10}, 01 \overline{10}))=i j \overline{10}$ for some two-bit sequence $i j$ and $g$ is continuous. Therefore, $g$ maps the first loop positively in the first coordinate.

Now, let $n=n_{0}+1$, and fix an even natural number $m$ such that

$$
\pi^{\prime}\left(g\left(01 e\left(S_{<h(n)}\right), 01 e^{\prime}\left(S_{<2 n}^{\prime}\right)\right)\right) \in i^{\prime} j^{\prime}\left[e^{\prime}(\varnothing), e^{\prime}\left(S_{<m}^{\prime}\right)\right)
$$

for some $i^{\prime}, j^{\prime} \in\{0,1\}$. Then, for any $N>n$,

$$
\begin{array}{r}
\pi^{\prime}\left(g\left(01 e\left(S_{<h(N)}\right), 01 e^{\prime}\left(S_{<2 N}^{\prime}\right)\right)\right), \pi^{\prime}\left(g\left(01 e\left(S_{<h(N+1)}\right), 01 e^{\prime}\left(S_{<2 N}^{\prime}\right)\right)\right) \\
\in i^{\prime} j^{\prime}\left[e^{\prime}(\varnothing), e^{\prime}\left(S_{<2(N-n)+m}^{\prime}\right)\right],
\end{array}
$$

but

$$
\pi\left(g\left(01 e\left(S_{<h(N+1)}\right), 01 e^{\prime}\left(S_{<2 N}^{\prime}\right)\right)\right) \notin i j\left[e(\varnothing), e\left(S_{<h(N+1)-h(N)-1}\right)\right)
$$

for any two-bit $i j$.

We know that $F$ " $A$ is compact; hence, for any $k$, the set of $z \in F$ " $A$ such that $\pi^{\prime}(z) \in i j\left[e^{\prime}(\varnothing), e^{\prime}\left(S_{<k}^{\prime}\right)\right]$ for some two-bit $i j$ is compact, so its image under $\pi$ is compact. Therefore, there is a number $\alpha(k) \in \omega$ such that every point in this image is in $i j\left[e(\varnothing), e\left(S_{<\alpha(k)}\right)\right)$ for some two-bit $i j$. If $\alpha(k)$ is taken to be the least such number, then $\alpha$ will be a nondecreasing function. It is easy to construct a strictly increasing function $h$ such that, for any $n$ and $m$, there is $N>n$ such that $h(N+1)>h(N)+\alpha(2(N-n)+m)$; in fact, we can simply take $h(N)$ to be $\sum_{n=0}^{N} \alpha(3 n)$. If $E$ is defined from this $h$, then the preceding paragraph shows that $g$ " $E$ cannot be contained in $F$ " $A$ for any suitable $g$, so $E$ has all of the required properties.

We will not try to prove more general results, such as something like "every countable lattice can be embedded into the monotone degrees so as to preserve joins and meets." In fact, this particular statement is false; the following proposition shows that any lattice embedded in this way is distributive. 
PROPOSITION 3.14. (a) If $C_{0}$ is a greatest lower bound (with respect to $\leq_{\mathrm{m}}$ ) of the monotone sets $A$ and $B_{0}$, and $C_{1}$ is a greatest lower bound of $A$ and $B_{1}$, then $\mathrm{jn}^{+}\left(C_{0}, C_{1}\right)$ is a greatest lower bound of $A$ and $\mathrm{jn}^{+}\left(B_{0}, B_{1}\right)$.

(b) If $C$ is a greatest lower bound of $B_{0}$ and $B_{1}$, then $\mathrm{jn}^{+}(A, C)$ is a greatest lower bound of $\mathrm{jn}^{+}\left(A, B_{0}\right)$ and $\mathrm{jn}^{+}\left(A, B_{1}\right)$.

Proof. (a) Clearly jn ${ }^{+}\left(C_{0}, C_{1}\right)$ is a lower bound. Suppose $\hat{A}$ is another lower bound. If $\hat{A}$ is $\varnothing$ or ${ }^{\omega} 2$, then $\hat{A}$ is jn ${ }^{+}$-prime, so from $\hat{A} \leq_{\mathrm{m}} \mathrm{jn}^{+}\left(B_{0}, B_{1}\right)$ we get $\hat{A} \leq_{\mathrm{m}} B_{0}$ or $\hat{A} \leq_{\mathrm{m}} B_{1}$, so $A \leq_{\mathrm{m}} C_{0}$ or $A \leq_{\mathrm{m}} C_{1}$. Otherwise, we can apply Proposition 2.3 to get sets $B_{0}^{\prime} \leq_{\mathrm{m}} B_{0}$ and $B_{1}^{\prime} \leq_{\mathrm{m}} B_{1}$ such that $\hat{A} \equiv_{\mathrm{m}} \mathrm{jn}^{+}\left(B_{0}^{\prime}, B_{1}^{\prime}\right)$; then $B_{0}^{\prime} \leq_{\mathrm{m}} C_{0}$ and $B_{1}^{\prime} \leq_{\mathrm{m}} C_{1}$, so $\hat{A} \leq_{\mathrm{m}} \mathrm{jn}^{+}\left(C_{0}, C_{1}\right)$.

(b) Again, $\mathrm{jn}^{+}(A, C)$ is clearly a lower bound, so suppose $\hat{A}$ is another lower bound. We are again done if $\hat{A}$ is $\varnothing$ or ${ }^{\omega} 2$ (since then either $\hat{A} \leq_{\mathrm{m}} A$ or both $\hat{A} \leq_{\mathrm{m}}$ $B_{0}$ and $\hat{A} \leq_{\mathrm{m}} B_{1}$ ), so assume otherwise. Then Proposition 2.3 gives monotone sets $A_{0}^{\prime}, A_{1}^{\prime} \leq_{\mathrm{m}} A, B_{0}^{\prime} \leq_{\mathrm{m}} B_{0}$, and $B_{1}^{\prime} \leq_{\mathrm{m}} B_{1}$ such that $\hat{A} \equiv_{\mathrm{m}} \mathrm{jn}^{+}\left(A_{0}^{\prime}, B_{0}^{\prime}\right) \equiv_{\mathrm{m}}$ $\mathrm{jn}^{+}\left(A_{1}^{\prime}, B_{1}^{\prime}\right)$. If $B_{0}^{\prime}$ is $\varnothing$ or ${ }^{\omega} 2$, then $B_{0}^{\prime} \leq_{\mathrm{m}} A_{1}^{\prime}$ or $B_{0}^{\prime} \leq_{\mathrm{m}} B_{1}^{\prime}$, so $\hat{A} \leq_{\mathrm{m}} \mathrm{jn}^{+}(A, C)$. Otherwise, there are $A_{1}^{\prime \prime} \leq_{\mathrm{m}} A_{1}^{\prime}$ and $B_{1}^{\prime \prime} \leq_{\mathrm{m}} B_{1}^{\prime}$ such that $B_{0}^{\prime} \equiv_{\mathrm{m}} \mathrm{jn}^{+}\left(A_{1}^{\prime \prime}, B_{1}^{\prime \prime}\right)$; then $B_{1}^{\prime \prime} \leq_{\mathrm{m}} C$, so $\hat{A} \leq_{\mathrm{m}} \mathrm{jn}^{+}(A, C)$.

We now turn to $\omega$-ary least upper bounds. When looking at Wadge reducibility, one finds two ways to put together an $\omega$-sequence of subsets of ${ }^{\omega} 2$ :

$$
\begin{aligned}
& \operatorname{jn}_{\omega}\left(\left\langle X_{n}: n \in \omega\right\rangle\right)=\bigcup_{n \in \omega} \operatorname{jn}\left(X_{0}, \operatorname{jn}\left(X_{1}, \ldots, j \mathrm{jn}\left(X_{n}, \varnothing\right) \cdots\right)\right), \\
& \overline{\mathrm{jn}}_{\omega}\left(\left\langle X_{n}: n \in \omega\right\rangle\right)=\bigcap_{n \in \omega} \operatorname{jn}\left(X_{0}, \mathrm{jn}\left(X_{1}, \ldots, \operatorname{jn}\left(X_{n},{ }^{\omega} 2\right) \cdots\right)\right) .
\end{aligned}
$$

These two sets differ only at the point $\overline{1}$. For the case of monotone reducibility, we can do something similar:

$$
\begin{aligned}
& \mathrm{jn}_{\omega}^{+}\left(\left\langle A_{n}: n \in \omega\right\rangle\right)=\bigcup_{n \in \omega} \mathrm{jn}^{+}\left(A_{0}, \mathrm{jn}^{+}\left(A_{1}, \ldots, \mathrm{jn}^{+}\left(A_{n}, \varnothing\right) \cdots\right)\right), \\
& \overline{\mathrm{jn}}_{\omega}^{+}\left(\left\langle A_{n}: n \in \omega\right\rangle\right)=\bigcap_{n \in \omega} \mathrm{jn}^{+}\left(A_{0}, \mathrm{jn}^{+}\left(A_{1}, \ldots, \mathrm{jn}^{+}\left(A_{n},{ }^{\omega} 2\right) \cdots\right)\right) .
\end{aligned}
$$

If we discard the trivial cases where $A_{n}=\varnothing$ for all but finitely many $n$ or $A_{n}={ }^{\omega_{2}}$ for all but finitely many $n$, then $\mathrm{jn}_{\omega}^{+}\left(\left\langle A_{n}: n \in \omega\right\rangle\right)$ and $\overline{\mathrm{jn}}_{\omega}^{+}\left(\left\langle A_{n}: n \in \omega\right\rangle\right)$ will differ only at the point $\overline{10}$. This difference, however, is critical, as the following proposition shows.

PROPOSITION 3.15. No strictly increasing $\omega$-sequence of monotone degrees has a least upper bound among the monotone degrees.

PROOF. If the sequence has no upper bound at all, it certainly has no least upper bound. Otherwise, we can choose a set from each of the degrees, so suppose we have monotone sets $A_{n}$ for $n \in \omega$ such that $A_{n}<_{\mathrm{m}} A_{n+1}$. Let

$$
B_{0}=\mathrm{jn}_{\omega}^{+}\left(\left\langle A_{n}: n \in \omega\right\rangle\right) \text { and } B_{1}=\overline{\mathrm{jn}}_{\omega}^{+}\left(\left\langle A_{n}: n \in \omega\right\rangle\right) .
$$

We will show that any $C$ such that $C \leq_{\mathrm{m}} B_{0}$ and $C \leq_{\mathrm{m}} B_{1}$ must satisfy $C \leq_{\mathrm{m}} A_{n}$ for some $n$; this clearly implies that $\left\{A_{n}: n \in \omega\right\}$ has no least upper bound. 
Suppose $f_{i}:{ }^{\omega} 2 \rightarrow{ }^{\omega} 2$ witnesses $C \leq_{\mathrm{m}} B_{i}$ for $i=0,1$. Clearly there is no $x$ such that $f_{0}(x)=f_{1}(x)=\overline{10}$; therefore, by compactness, there is $N \in \omega$ such that for no $x \in \omega^{\omega_{2}}$ do we have $f_{0}(x) \uparrow(2 N)=f_{1}(x) \uparrow(2 N)=(10)^{N}$. For any $x \in \operatorname{Fr}(C)$ and $i<2$, either $f_{i}(x)=\overline{10}$ or $f_{i}(x) \in(10)^{n} 01^{\omega} 2$ for some $n \in \omega$; let $h_{i}(x)$ be $\omega$ in the former case and $n$ in the latter. For any such $x$, either $h_{0}(x)<N$ or $h_{1}(x)<N$.

For each $n<N$, choose a function $g_{n}:{ }^{\omega} 2 \rightarrow{ }^{\omega} 2$ witnessing $A_{n} \leq_{\mathrm{m}} A_{N}$. Now define $f: \operatorname{Fr}(C) \rightarrow{ }^{\omega_{2}}$ by: if $h_{0}(x) \leq h_{1}(x)$, let $f(x)=g_{h_{0}(x)}(y)$, where $f_{0}(x)=$ $(10)^{h_{0}(x)} 01 y$; otherwise, let $f(x)=g_{h_{1}(x)}(y)$, where $f_{1}(x)=(10)^{h_{1}(x)} 01 y$. It is easy to verify that $f$ satisfies the hypotheses of Proposition 2.2, so $C \leq_{\mathrm{m}} A_{N}$.

This solves the problem of deciding whether a countable collection of degrees has a least upper bound; it does if and only if the least upper bound of some finite subcollection is an upper bound for the whole collection. If we restrict ourselves to $\mathcal{F}_{\rho}$ degrees and ask whether a countable collection of them has a least upper bound among the $\mathcal{F}_{\rho}$ degrees, however, the answer may be different. If $A_{n}$ for $n \in \omega$ are monotone $\mathcal{F}_{\rho}$ sets, then $\mathrm{jn}_{\omega}^{+}\left(\left\langle A_{n}: n \in \omega\right\rangle\right)$ will also be $\mathcal{F}_{\rho}$, but $\overline{\mathrm{jn}}_{\omega}^{+}\left(\left\langle A_{n}: n \in \omega\right\rangle\right)$ will not (unless $A_{n}$ is closed for all but finitely many $n$ ). Therefore, the set $\mathrm{jn}_{\omega}^{+}\left(\left\langle A_{n}: n \in \omega\right\rangle\right)$ is a reasonable candidate for a least $\mathcal{F}_{\rho}$ upper bound of $\left\{A_{n}: n \in\right.$ $\omega\}$. In fact, we find that it does work in some cases, but not in others.

Call a monotone set $A$ local iff there is a point $x \in A$ such that, for all $n \in \omega$, $A \leq_{\mathrm{m}}\left\{y \in{ }^{\omega} 2:(x \mid n) y \in A\right\}$. It is easy to show that, if $A$ is local and $B \equiv_{\mathrm{m}} A$, then $B$ is local; so call a monotone degree local iff its members are local. Clearly any local set is $\mathrm{jn}^{+}$-prime, but the converse fails; the set in Figure 2.2(b) is not local, even though its frontier is m-connected. An example of a local set is $\mathrm{jn}_{\omega}^{+}\left(\left\langle A_{n}: n \in \omega\right\rangle\right)$ where $A_{n} \leq_{\mathrm{m}} A_{n+1}$ for all $n$.

PROPOSITION 3.16. If $A_{n}$ for $n \in \omega$ are local $\mathcal{F}_{\rho}$ monotone sets, and $A_{n}<_{\mathrm{m}}$ $A_{n+1}$ for all $n$, then $\mathrm{jn}_{\omega}^{+}\left(\left\langle A_{n}: n \in \omega\right\rangle\right)$ is a least monotone $\mathcal{F}_{\rho}$ upper bound for $\left\{A_{n}: n \in \omega\right\}$.

PROOF. Suppose $C$ is a monotone $\mathcal{F}_{\rho}$ upper bound for this collection. For each $n$, choose a point $x_{n}$ witnessing that $A_{n}$ is local, and choose a function $f_{n}:{ }^{\omega} 2 \rightarrow{ }^{\omega} 2$ witnessing $A_{n} \leq_{\mathrm{m}} C$. Since ${ }^{\omega} 2$ is compact, there is a strictly increasing $h: \omega \rightarrow \omega$ such that $\left\langle f_{h(n)}\left(x_{h(n)}\right): n \in \omega\right\rangle$ converges to some point $z$. Since $A_{h(n)}$ is local at $x_{h(n)}$, there is a function $g_{n}:{ }^{\omega} 2 \rightarrow{ }^{\omega} 2$ witnessing $A_{h(n)} \leq_{\mathrm{m}} A_{h(n)}$ such that $g$ " $\omega_{2}$ is contained in the neighborhood $\left\{x: f_{h(n)}(x)\left|n=f_{h(n)}\left(x_{h(n)}\right)\right| n\right\}$ of $x_{h(n)}$. Choose $\tilde{g}_{n}:{ }^{\omega} 2 \rightarrow{ }^{\omega} 2$ witnessing $A_{n} \leq_{\mathrm{m}} A_{h(n)}$. Now define

$$
F: \operatorname{Fr}\left(\mathrm{jn}_{\omega}^{+}\left(\left\langle A_{n}: n \in \omega\right\rangle\right)\right) \rightarrow{ }^{\omega_{2}}
$$

by $F(\overline{10})=z$ and, for any $x$ in the domain of $F$ other than $\overline{10}, F(x)=$ $f_{h(n)}\left(g_{n}\left(\tilde{g}_{n}\left(x^{\prime}\right)\right)\right)$, where $n$ and $x^{\prime}$ are chosen to satisfy $x=(10)^{n} 01 x^{\prime}$. Since $A_{n} \neq{ }^{\omega} 2$ for $n>0, \overline{10} \notin \mathrm{jn}_{\omega}^{+}\left(\left\langle A_{n}: n \in \omega\right\rangle\right)$; since $A_{n}$ is not closed for $n>2$, the choice of the functions $g_{n}$ guarantees that there are points of $\operatorname{Fr}(C)-C$ arbitrarily close to $z$, so, since $C$ is $\mathcal{F}_{\rho}, z \notin C$. It is now easy to verify that $F$ satisfies

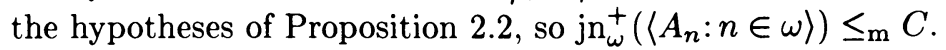

On the other hand:

PROPOSITION 3.17. If $\left\{A_{n}: n \in \omega\right\}$ is an infinite antichain of monotone $\mathcal{F}_{\rho}$ sets with m-connected frontiers, then it has no least $\mathcal{F}_{\rho}$ upper bound. 
ProOF. We may assume that $A_{i} \neq A_{j}$ for $i \neq j$ (in fact, you probably already did). Suppose $B$ is a least monotone $\mathcal{F}_{\rho}$ upper bound for $\left\{A_{n}: n \in \omega\right\}$. For each $n$, choose a point $x_{n} \in \operatorname{Fr}\left(A_{n}\right)$ and a function $f_{n}$ witnessing $A_{n} \leq_{\mathrm{m}} B$. By compactness, there is an infinite set $W \subseteq \omega$ such that $\left\langle f_{n}\left(x_{n}\right): n \in W\right\rangle$ converges to some point $z$. Choose $V \subset W$ so that $V$ and $W-V$ are both infinite. Now let $C=\mathrm{jn}^{+}\left(\mathrm{jn}_{\omega}^{+}\left(\left\langle A_{n}^{0}: n \in \omega\right\rangle\right), \mathrm{jn}_{\omega}^{+}\left(\left\langle A_{n}^{1}: n \in \omega\right\rangle\right)\right)$, where $A_{n}^{0}=A_{n}$ and $A_{n}^{1}=\varnothing$ if $n \in V$, but $A_{n}^{0}=\varnothing$ and $A_{n}^{1}=A_{n}$ if $n \notin V$. Clearly $A_{n} \leq_{\mathrm{m}} C$ for all $n$, so there is $f:{ }^{\omega_{2}} \rightarrow{ }^{\omega_{2}}$ witnessing $B \leq_{\mathrm{m}} C$. Since $z \in \operatorname{Fr}(B), f(z) \in \operatorname{Fr}(C)$, so $f(z)\lceil 2$ is either 01 or 10. Assume it is 01 ; the other case is similar. Choose $n \in W-V$ so large that $f\left(f_{n}\left(x_{n}\right)\right)\left\lceil 2=01\right.$. Since the frontier of $A_{n}$ is m-connected, $\left(f \circ f_{n}\right)$ " $\operatorname{Fr}\left(A_{n}\right) \subseteq 01^{\omega} 2$. For any $m, \operatorname{Fr}(C) \cap 01(10)^{m} 01^{\omega} 2$ and $\operatorname{Fr}(C)-01(10)^{m} 01^{\omega} 2$ are m-disjoint closed sets; hence, if $f\left(f_{n}(x)\right)$ were in $01(10)^{m} 01^{\omega} 2$ for some $x \in \operatorname{Fr}\left(A_{n}\right)$, we would have $\left(f \circ f_{n}\right)$ " $\operatorname{Fr}\left(A_{n}\right) \subseteq 01(10)^{m} 01^{\omega} 2$, which would imply $A_{n} \leq_{\mathrm{m}} A_{m}^{0}$, which is impossible since $A_{n}^{0}=\varnothing$ and $A_{n} \mathbb{Z}_{\mathrm{m}} A_{m}$ for $m \neq n$. The only remaining possibility is $f\left(f_{n}(x)\right)=01 \overline{10}$ for all $x \in \operatorname{Fr}\left(A_{n}\right)$, which cannot happen because $A_{n}$ is not open. Therefore, we have a contradiction.

3. Lifting Hausdorff hierarchy results to higher degrees. We have seen that the collection of monotone degrees in the Wadge degree of strictly $\mathcal{F}_{\rho}$ sets is quite complicated. The same applies to $\mathcal{F}_{\rho-}$ by duality, but it does not seem to apply immediately to higher Wadge degrees. The next two propositions allow us to lift results at the $\mathcal{F}_{\rho}$ level to any higher Wadge degree.

Recall the notations $\mathrm{Ha}_{\alpha}$ and $R_{\alpha}$ introduced at the beginning of part III; since we will be working with various sets $X$, we use $R_{\alpha}(X)$ to denote the $R_{\alpha}$ obtained from $X$. In particular, $R_{1}(X)=\operatorname{Fr}(X)$.

Proposition 3.18. (a) If $A$ is either $\Sigma_{2}^{0}$ or $\Pi_{2}^{0}$, and $A \leq_{\mathrm{m}} X_{\mathrm{b}}$ for some set $X \subseteq{ }^{\omega} 2$, then $A \leq_{\mathrm{m}} A_{\mathrm{b}}$.

(b) If $A \equiv_{\mathrm{w}} T(\delta \sigma H)$ for some $\omega$-ary Boolean operation $H$, and $A \leq_{\mathrm{m}} X_{\mathrm{b}}$ for some set $X \subseteq{ }^{\omega} 2$, then $A \leq_{\mathrm{m}} A_{\mathrm{b}}$.

(Recall that $T(H)$ is the truth table of $H$, and that $H_{0} H_{1}$ means first apply $H_{0}$, then apply $H_{1}$.)

ProOF. (a) This is trivial if $A$ is clopen, so assume $\operatorname{Fr}(A) \neq \varnothing$. Clearly $\operatorname{Fr}\left(X_{b}\right) \subseteq f_{b}$ " $\omega_{2}$. In fact, we may assume that $\operatorname{Fr}\left(X_{b}\right)$ is nowhere dense in $f_{b} " \omega_{2}$. (Let $\tilde{g}$ be any continuous one-to-one map from ${ }^{\omega_{2}}$ to a nowhere dense subset of ${ }^{\omega_{2}}$, and let $\tilde{X}=\tilde{g}$ " $X$; then $X \leq_{\mathrm{w}} \tilde{X}$ and $\operatorname{Fr}(\tilde{X})$ is nowhere dense in ${ }^{{ }^{2}} 2$, so $X_{\mathrm{b}} \leq_{\mathrm{m}} \tilde{X}_{\mathrm{b}}$ and $\operatorname{Fr}\left(\tilde{X}_{b}\right)$ is nowhere dense in $f_{b} " \omega_{2}$.)

We will be using the analogue for $\leq_{\mathrm{w}}$ of Proposition 2.1: for any closed nowhere dense $S \subseteq \omega_{2}$ and any $T \subseteq S$, there is a set $Y \subseteq \omega_{2}$ such that $\operatorname{Fr}(Y)=S$ and $S \cap Y=T$. This is easier than Proposition 2.1; simply define $Y$ by: if $y \in S$, then $y \in Y$ iff $y \in T$; if $y \notin S$, let $n \in \omega$ be least such that $\left(y\lceil n)^{\omega} 2 \cap S=\varnothing\right.$, and put $y$ in $Y$ iff $y(n)=1$.

Suppose $f:{ }^{\omega_{2}} \rightarrow{ }^{\omega_{2}}$ witnesses $A \leq_{\mathrm{m}} X_{b}$; then $f$ " $\operatorname{Fr}(A) \subseteq \operatorname{Fr}\left(X_{b}\right)$. Define $g: \operatorname{Fr}(A) \rightarrow{ }^{\omega_{2}}$ by $g(x)=f_{b}{ }^{-1}(f(x))$, and let $Y$ be as above for $S=g$ " $\operatorname{Fr}(A)$ and $T=g$ " $(\operatorname{Fr}(A) \cap A)$. Then, for any $x \in f$ " $\operatorname{Fr}(A), x \in \operatorname{Fr}\left(Y_{\mathrm{b}}\right)$, and $x \in Y_{\mathrm{b}}$ iff $x \in X_{\mathrm{b}}$, so Proposition 2.2 gives $A \leq_{\mathrm{m}} Y_{\mathrm{b}}$. We will now show $Y \equiv_{\mathrm{w}} A$; this implies $Y_{\mathrm{b}} \leq_{\mathrm{m}} A_{\mathrm{b}}$ and hence $A \leq_{\mathrm{m}} A_{\mathrm{b}}$. 
To prove that $Y \equiv_{\mathrm{w}} A$, it will suffice to prove that, for all $\alpha>0, R_{\alpha}(Y)=$ $g$ " $R_{\alpha}(A)$. If this holds, then since $g(x) \in Y$ iff $x \in A$ for $x \in \operatorname{Fr}(A), R_{\alpha}(Y) \cap Y=$ $g$ " $\left(R_{\alpha}(A) \cap A\right)$ and $R_{\alpha}(Y)-Y=g$ " $\left(R_{\alpha}(A)-A\right)$ for $\alpha>0$, so $Y \in \mathrm{Ha}_{\alpha}$ iff $A \in \mathrm{Ha}_{\alpha}$ and $Y \in \mathrm{Ha}_{\alpha-}$ iff $X \in \mathrm{Ha}_{\alpha-}$ for $\alpha>0$, so $A$ and $Y$ must be in the same Wadge degree if either of them is $\Delta_{2}^{0}$ (since $A$ is not clopen). If $A$ is strictly $\Sigma_{2}^{0}$, then $\operatorname{Fr}(A) \cap A$ is a countable union of compact sets, so $g$ " $(\operatorname{Fr}(A) \cap A)$ is a countable union of compact sets, so $Y$ is $\Sigma_{2}^{0}$; by working with $\operatorname{Fr}(A)-A$ instead, we see that $Y$ is $\Pi_{2}^{0}$ if $A$ is. The result now follows from the well-known fact that any two strictly $\Sigma_{2}^{0}$ sets are Wadge equivalent. (This fact follows from determinacy of $\left(\Sigma_{2}^{0}\right)_{\rho \text { - }}$ sets via Wadge's Lemma, but there is a simpler proof; since I do not know of a reference for it, I will give it here. It will suffice to show that, for any strictly $\Sigma_{2}^{0}$ set $S, T(\delta \sigma) \leq_{\mathrm{m}} S$, or, equivalently, there is a continuous map $F:{ }^{\omega \times \omega_{2}} \rightarrow{ }^{\omega_{2}}$ such that $F^{-1}$ " $S=\{x: \exists m \forall k x(m, k)=1\}$. If $S$ is strictly $\Sigma_{2}^{0}$, then $R_{\alpha}(S) \neq \varnothing$ for all $\alpha$, so there is an $\alpha$ such that $R_{\alpha}(S)=R_{\alpha+1}(S) \neq \varnothing$; then $S \cap R_{\alpha}(S)$ is dense and codense in the closed set $R_{\alpha}(S)$. Suppose $S$ is the union of the closed sets $S_{n}$ $(n \in \omega)$. For each basic open set $N$ meeting $R_{\alpha}(S)$ and each $n \in \omega$, one can choose a point $x(N, n) \in N \cap S \cap R_{\alpha}(S)$ and basic open sets $U(N, n, k)$ for $k \in \omega$ such that $\overline{U(N, n, k)} \subseteq N, U(N, n, k)$ meets $R_{\alpha}(S)$ but not $S_{n}$, the diameter of $U(N, n, k)$ in the usual metric for ${ }^{\omega_{2}}$ is at most $2^{-n}$, and every neighborhood of $x(N, n)$ contains $U(N, n, k)$ for all sufficiently large $k$. Define $x_{s}$ and $N_{s}$ for $s \in<\omega \omega$ as follows: $N_{\langle\rangle}=\omega_{2}$; given $N_{s}$, let $x_{s}=x\left(N_{s}, \ell(s)\right)$ and $N_{s k}=U\left(N_{s}, \ell(s), k\right)$ for $k \in \omega$. Now we can define $F$. For any $x \in{ }^{\omega \times \omega} 2$, let $h(m)$ be the least $k$ such that $x(m, k)=0$ for any $m$ such that such a $k$ exists. If $h(m)$ exists for all $m$, define $F(x)$ to be the unique point in $\bigcap_{n \in \omega} N_{h\lceil n}$; otherwise, let $m$ be least such that $h(m)$ does not exist, and let $F(x)=x_{h \uparrow m}$. It is not hard to show that $F$ is well-defined and has the required properties.)

We prove $R_{\alpha}(Y)=g$ " $R_{\alpha}(A)$ by induction on $\alpha>0$. For $\alpha=1$ it follows from the definition of $Y$. The limit case is a simple compactness argument. If it is true for $\alpha$, then $R_{\alpha}(Y) \cap Y=g$ " $\left(R_{\alpha}(A) \cap A\right)$ and $R_{\alpha}(Y)-Y=g$ " $\left(R_{\alpha}(A)-A\right)$, so $\overline{R_{\alpha}(Y) \cap Y} \supseteq g$ " $\overline{R_{\alpha}(A) \cap A}$ and $\overline{R_{\alpha}(Y)-Y} \supseteq g$ " $\overline{R_{\alpha}(A)-A}$ since $g$ is continuous, so $R_{\alpha+1}(Y) \supseteq g$ " $R_{\alpha+1}(A)$. Now, suppose $y \in R_{\alpha+1}(Y)$. Choose a sequence of points $y_{n}$ such that $y_{n} \rightarrow y$ as $n \rightarrow \infty, y_{n} \in R_{\alpha}(Y)$, and $y_{n} \in Y$ iff $y \notin Y$. For each $n$, choose $x_{n} \in R_{\alpha}(A)$ such that $g\left(x_{n}\right)=y_{n}$. By compactness, some subsequence of $\left\langle x_{n}: n \in \omega\right\rangle$ converges to a point $x$; clearly $g(x)=y$. Then $x_{n} \in A$ iff $g\left(x_{n}\right) \in Y$ iff $y \notin Y$ iff $x \notin A$, so $x \in R_{\alpha+1}(A)$. Therefore, $y \in g$ " $R_{\alpha+1}(A)$, so we are done.

(b) The proof is the same, except that here we need a different method for showing $Y \leq_{\mathrm{w}} A\left(A \leq_{\mathrm{w}} Y\right.$ follows from $\left.A \leq_{\mathrm{m}} Y_{\mathrm{b}}\right)$. To do this, we will use a result of Louveau and Saint Raymond [10, $\S 4$, Corollary 2]; the case we need can be stated as follows:

If $H$ is an $\omega$-ary Boolean operation, $\chi$ and $y$ are compact metrizable spaces, $\pi: \mathcal{X} \rightarrow \mathcal{Y}$ is a continuous surjection, $S \subseteq \mathcal{Y}$, and $\pi^{-1}$ " $S$ is in $\left(\Sigma_{2}^{0}(\chi)\right)_{H}$ (where $\Sigma_{2}^{0}(\mathcal{X})$ is the collection of $\mathcal{F}_{\sigma}$ subsets of $\left.\mathcal{X}\right)$, then $S$ is in $\left(\Sigma_{2}^{0}(\mathcal{Y})\right)_{H}$.

Since $A \leq_{\mathrm{w}} T(\delta \sigma H), A$ is in $\mathrm{Cp}_{\delta \sigma H}=\left(\Sigma_{2}^{0}\right)_{H}$, so $A \cap \operatorname{Fr}(A)$ is in $\left(\Sigma_{2}^{0}(\operatorname{Fr}(A))\right)_{H}$. We now apply the result quoted above with $\chi=\operatorname{Fr}(A), \mathcal{Y}=\operatorname{Fr}(Y), \pi=g$, and $S=Y \cap \operatorname{Fr}(Y)$ to show that $Y \cap \operatorname{Fr}(Y)$ is in $\left(\Sigma_{2}^{0}(\operatorname{Fr}(Y))\right)_{H}$. Suppose $Y \cap \operatorname{Fr}(Y)=$ $H\left(\left\langle E_{n}: n \in \omega\right\rangle\right)$, where the sets $E_{n}$ are $\Sigma_{2}^{0}$ subsets of $\operatorname{Fr}(Y)$. Since $A$ is not clopen, $H$ cannot be one of the two trivial operations (i.e., $T(H)$ is neither $\varnothing$ nor ${ }^{\omega_{2}}$ ), so 
there is a point $z \in{ }^{\omega} 2$ such that $z \in T(H)$ iff $\overline{0} \notin T(H)$. Let $\tilde{E}$ be $Y-\operatorname{Fr}(Y)$ if $z \in T(H)$ and $(-Y)-\operatorname{Fr}(Y)$ otherwise, and let $E_{n}^{\prime}$ be $E_{n} \cup \tilde{E}$ if $z(n)=1$, $E_{n}$ otherwise. Since $E_{n}$ is $\Sigma_{2}^{0}$ relative to a closed subset of ${ }^{\omega_{2}} 2, E_{n}$ is $\Sigma_{2}^{0}$ in ${ }^{\omega_{2}}$; since $\tilde{E}$ is open, $E_{n}^{\prime}$ is $\Sigma_{2}^{0}$. It is easy to see that $Y=H\left(\left\langle E_{n}^{\prime}: n \in \omega\right\rangle\right)$, so $Y$ is in $\left(\Sigma_{2}^{0}\right)_{H}=\mathrm{Cp}_{\delta \sigma H}$, so $Y \leq_{\mathrm{w}} T(\delta \sigma H) \leq_{\mathrm{w}} A$.

Among the $A$ 's to which $3.18(\mathrm{~b})$ applies are the $\Sigma_{\alpha}^{0}$-complete sets and the $\Pi_{\alpha}^{0}$ complete sets for $\alpha \geq 2$, and the $\Sigma_{n}^{1}$-complete sets and the $\Pi_{n}^{1}$-complete sets for $n \geq 1$.

It would be nice if this proposition were true for arbitrary $A$, since then the 'flat' degrees would form a downward closed collection of monotone degrees isomorphic to the Wadge degrees under $\leq_{w}$. However, it fails for $A$ in the Wadge degree which is the least upper bound of the degree of strictly $\Sigma_{2}^{0}$ sets and the degree of strictly $\Pi_{2}^{0}$ sets. A set $X \subseteq{ }^{\omega} 2$ is in this degree iff $X$ is neither $\boldsymbol{\Sigma}_{2}^{0}$ nor $\Pi_{2}^{0}$, but there is a clopen set $Y$ such that $X \cap Y$ is $\Sigma_{2}^{0}$ and $X-Y$ is $\Pi_{2}^{0}$. Now, fix a strictly $\Sigma_{2}^{0}$ set $Y$ and a strictly $\Pi_{2}^{0}$ set $\tilde{Y}$, and define $A$ and $X$ by

$$
\begin{aligned}
& A=\mathrm{jn}\left(\mathrm{jn}_{\omega}^{+}\left(Y_{b}, \varnothing, Y_{b}, \varnothing, \ldots\right), \mathrm{jn}_{\omega}^{+}\left({ }^{\omega} 2, \tilde{Y}_{b},{ }^{\omega} 2, \tilde{Y}_{b}, \ldots\right)\right), \\
& X=\operatorname{jn}_{\omega}(Y, \tilde{Y}, Y, \tilde{Y}, \ldots) .
\end{aligned}
$$

(See Figure 3.7.) Then $A \leq_{\mathrm{m}} X_{\mathrm{b}}$. Now, suppose $A \leq_{\mathrm{m}} A_{\mathrm{b}}$, and let $f$ be a witness to this. Since $f$ maps frontier points of $A$ to frontier points of $A_{b}$, and any two distinct frontier points of $A_{b}$ are incomparable, we must have $f(0 \overline{10})=f(1 \overline{10})$. But then there is no neighborhood $N$ of $f(0 \overline{10})$ such that $A_{b} \cap N$ is either $\Sigma_{2}^{0}$ or $\Pi_{2}^{0}$, so $A_{\mathrm{b}} \mathbb{Z}_{\mathrm{w}} A$, contradiction.

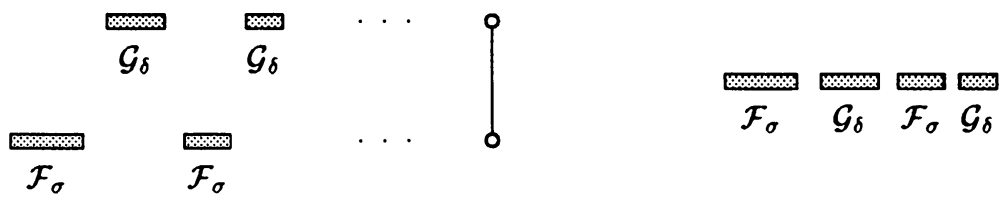

$A$

$X_{\mathrm{b}}$

FIgURE 3.7 . A case where $A \leq_{\mathrm{m}} X_{\mathrm{b}}$ but $A \Varangle_{\mathrm{m}} A_{\mathrm{b}}$

PROPOSITION 3.19. If $A$ and $B$ are monotone sets such that $A$ is either $\Sigma_{2}^{0}$ or $\Pi_{2}^{0}, A \leq_{\mathrm{w}} B$, and $A \underline{Z}_{\mathrm{m}} B$, then $A \mathbb{Z}_{\mathrm{m}} \mathrm{jn}^{+}\left(B, X_{\mathrm{b}}\right)$ for all $X \subseteq{ }^{\omega} 2$.

Proof. Suppose $A \leq_{\mathrm{m}} \mathrm{jn}^{+}\left(B, X_{\mathrm{b}}\right)$. $A$ cannot be $\varnothing$ or ${ }^{\omega} 2$, since otherwise $A \leq_{\mathrm{w}}$ $B$ would imply $A \leq_{\mathrm{m}} B$. By Proposition $2.3, A \equiv_{\mathrm{m}} \mathrm{jn}^{+}\left(B^{\prime}, C\right)$ for some $B^{\prime} \leq_{\mathrm{m}} B$ and $C \leq_{\mathrm{m}} X_{\mathrm{b}}$. Since $C \leq_{\mathrm{m}} X_{\mathrm{b}}, C \leq_{\mathrm{m}} C_{\mathrm{b}}$ by $3.18(\mathrm{a}) ;$ but $C_{\mathrm{b}} \leq_{\mathrm{m}} A_{\mathrm{b}} \leq_{\mathrm{m}} B_{\mathrm{b}} \leq_{\mathrm{m}} B$, so $A \leq_{\mathrm{m}} B$, contradiction.

Proposition 3.18(a) is the special case of Proposition 3.19 with $B=A_{\mathrm{b}}$. The corresponding extension of 3.18(b) does not hold without the extra assumption that $A$ is $\mathrm{jn}^{+}$-prime, in which case it says no more than 3.18(b) anyway.

Proposition 3.19 allows us to lift existence results from a $\Sigma_{2}^{0}$ or $\Pi_{2}^{0}$ Wadge degree to any higher Wadge degree. Suppose $d_{0}<_{\mathrm{w}} d_{1}$ are Wadge degrees and $d_{0}$ consists of $\Sigma_{2}^{0}$ or $\Pi_{2}^{0}$ sets. Then we can map $d_{0} \cap$ Mon into $d_{1} \cap$ Mon by sending $A \in d_{0}$ to $\mathrm{jn}^{+}\left(A, X_{\mathrm{b}}\right)$ for some fixed $X \in d_{1}$. If $I$ is this mapping, then clearly $A \leq_{\mathrm{m}} B$ implies $I(A) \leq_{\mathrm{m}} I(B)$; Proposition 3.19 states that $A \not_{\mathrm{m}} B$ implies $I(A) \mathbb{Z}_{\mathrm{m}} I(B)$. 
Therefore, $I$ gives an embedding of the monotone degrees in $d_{0}$ into the monotone degrees in $d_{1}$. In particular, if $d_{1}$ is any Wadge degree above the degree of strictly $\mathcal{F}_{\rho}$ sets, then $d_{1}$ contains at least $2^{\aleph_{0}}$ monotone degrees, and arbitrary countable partial orderings or semilattices (since $I$ clearly preserves $\mathrm{jn}^{+}$up to $\equiv_{\mathrm{m}}$ ) can be embedded into the monotone degrees in $d_{1}$. Furthermore, Proposition 3.14(b) implies that $I$ preserves (binary) greatest lower bounds whenever they exist in $d_{0}$.

This method does not, in general, allow us to lift nonexistence results; however, $\mathrm{jn}^{+}$-primeness can sometimes be used to get around the problem. For example, if the embedding $I$ above is applied to the sets in Proposition 3.13, the resulting sets will still have no greatest lower bound. To see this, suppose $B_{0}$ and $B_{1}$ are the two $\mathcal{F}_{\rho}$ sets, and $A$ is a nonclopen lower bound for $\mathrm{jn}^{+}\left(B_{0}, X_{\mathrm{b}}\right)$ and $\mathrm{jn}^{+}\left(B_{1}, X_{\mathrm{b}}\right)$. Apply Proposition 2.3 to get $B_{0}^{\prime} \leq_{\mathrm{m}} B_{0}, B_{1}^{\prime} \leq_{\mathrm{m}} B_{1}$, and $C_{0}, C_{1} \leq_{\mathrm{m}} X_{\mathrm{b}}$ such that $A \equiv_{\mathrm{m}} \mathrm{jn}^{+}\left(B_{0}^{\prime}, C_{0}\right)$ and $B_{0}^{\prime} \equiv_{\mathrm{m}} \mathrm{jn}^{+}\left(B_{1}^{\prime}, C_{1}\right)$; then $B_{1}^{\prime}$ is a lower bound for $B_{0}$ and $B_{1}$. The proof of 3.13 showed that there is a strictly $\mathcal{F}_{\rho}$ set $C$ such that $C \leq_{\mathrm{m}} B_{0}$, $C \leq_{\mathrm{m}} B_{1}, C \mathbb{Z}_{\mathrm{m}} C_{\mathrm{b}}$, the frontier of $C$ is m-connected, and $C \mathbb{Z}_{\mathrm{m}} B_{1}^{\prime}$. Then $C \mathbb{Z}_{\mathrm{m}} X_{\mathrm{b}}$ by 3.18(a), so $C \mathbb{Z}_{\mathrm{m}} B_{0}^{\prime}$ and $C \mathbb{Z}_{\mathrm{m}} A$ since $C$ is jn ${ }^{+}$-prime; therefore, $A$ is not a greatest lower bound for $\mathrm{jn}^{+}\left(B_{0}, X_{\mathrm{b}}\right)$ and $\mathrm{jn}^{+}\left(B_{1}, X_{\mathrm{b}}\right)$.

One can also show that, if $S$ is a collection of monotone sets in $d_{0}$ which has a monotone upper bound in $d_{0}$ but no least monotone upper bound in $d_{0}$, then $I$ " $S$ has no least monotone upper bound in $d_{1}$. (This lets us lift Proposition 3.17 to higher degrees.) To see this, suppose $B \in d_{0}$ is an upper bound for $S$ and $A \in d_{1}$ is a least monotone upper bound for $I$ " $S$ in $d_{1}$. Then $A \leq_{\mathrm{m}} I(B)$, so by $2.3 A \equiv_{\mathrm{m}} \mathrm{jn}^{+}\left(B^{\prime}, C\right)$ for some $B^{\prime} \leq_{\mathrm{m}} B$ and $C \leq_{\mathrm{m}} X_{\mathrm{b}}$. Choose a fixed $B_{0} \in S$. It follows from 3.19 that $\mathrm{jn}^{+}\left(B^{\prime}, B_{0}\right)$ is an upper bound for $S$, so there is another upper bound $\tilde{B} \in d_{0}$ for $S$ such that $\mathrm{jn}^{+}\left(B^{\prime}, B_{0}\right) Z_{\mathrm{m}} \tilde{B}$. By 3.19 again, $I\left(\mathrm{jn}^{+}\left(B^{\prime}, B_{0}\right)\right) \not_{\mathrm{m}} I(\tilde{B})$; but clearly $B_{0} \leq_{\mathrm{m}} I(\tilde{B})$ and $X_{\mathrm{b}} \leq_{\mathrm{m}} I(\tilde{B})$, so $B^{\prime} \underline{Z}_{\mathrm{m}} I(\tilde{B})$. Therefore, $A \mathbb{Z}_{\mathrm{m}} I(\tilde{B})$, which is a contradiction since $I(\tilde{B})$ is an upper bound for $I$ " $S$.

4. The rest of the Hausdorff hierarchy. In the remainder of this paper, we will look at the best-known Wadge degrees above the degree of strictly $\mathcal{F}_{\rho}$ sets. Preceding sections have given existence results for these degrees, so from now on we will look at structural questions: the relationship between 'monotone' and 'positive,' and the polarized separation and reduction properties.

We start by defining a notion of 'positive' for sets in $\mathrm{Ha}_{\alpha}$. To do this, we look at the alternating series representation for $\Delta_{2}^{0}$ sets: for any $\Delta_{2}^{0}$ set $X$, there is a countable ordinal $\gamma$ and a sequence $\left\langle E_{\alpha}: \alpha<\gamma\right\rangle$ of closed sets such that $E_{\beta} \supseteq E_{\alpha}$ for $\beta<\alpha$ and $X$ can be expressed as the alternating series $E_{0}-E_{1} \cup E_{2}-E_{3} \cup \cdots$ of length $\gamma$. In other words, a point $x$ is in $X$ iff there is a greatest $\alpha$ such that $x \in E_{\alpha}$ and this $\alpha$ is even. (An ordinal is even iff its successor part is even; statements such as " $\alpha$ and $\beta$ have the same parity" are interpreted similarly.) Furthermore, if we fix $\gamma<\omega_{1}$ and let $S$ be the collection of all $X$ that can be expressed in such a form for the given $\gamma$, then $S$ will be $\mathrm{Ha}_{\gamma}$ if $\gamma$ is even and $\mathrm{Ha}_{\gamma-}$ otherwise. (This is due to Hausdorff $[4, \S 30]$. To prove it, note that, for $X$ in the above form, $R_{\alpha}(X) \cap X \subseteq E_{\alpha}$ if $\alpha$ is even and $R_{\alpha}(X)-X \subseteq E_{\alpha}$ if $\alpha$ is odd, while if $X$ is in $\mathrm{Ha}_{\gamma}$ for even $\gamma$ or $\mathrm{Ha}_{\gamma-}$ for odd $\gamma$, we can let $E_{\alpha}$ be $\overline{R_{\alpha}(X) \cap X}$ if $\alpha$ is even and $\overline{R_{\alpha}(X)-X}$ if $\alpha$ is odd.) 
In other words, a set $X$ is in $\mathrm{Ha}_{\gamma}$ iff there are closed sets $E_{\alpha}$ for $\alpha<\gamma$ such that $E_{\beta} \supseteq E_{\alpha}$ for $\beta<\alpha$, and a point $x$ is in $X$ iff the least $\alpha<\gamma$ such that $x \notin E_{\alpha}$ exists and has opposite parity from $\gamma$. In this form, we see that the restriction that $E_{\beta} \supseteq E_{\alpha}$ for $\beta<\alpha$ is unnecessary, since if we define $X$ as above from an arbitrary sequence $\left\langle E_{\alpha}: \alpha<\gamma\right\rangle$ of closed sets, it will be the same as if we had defined $X$ from $\left\langle\bigcap_{\beta \leq \alpha} E_{\beta}: \alpha<\gamma\right\rangle$. Therefore, we have expressed $\mathrm{Ha}_{\gamma}$ as $\mathcal{F}_{H}=\mathrm{Cp}_{\delta H}$ for a $\gamma$-ary operation $H$ which is clearly Boolean. The operation $H$ is not monotone as it stands, but it is monotone if considered as an operation on $\left\langle E_{\alpha}^{\prime}: \alpha<\gamma\right\rangle$, where $E_{\alpha}^{\prime}$ is $E_{\alpha}$ if $\alpha$ and $\gamma$ have the same parity, $-E_{\alpha}$ otherwise. We therefore arrive at a definition of 'positive' for $\mathrm{Ha}_{\gamma}$ sets by applying this operation to positive sets of the right type.

DEFINITION. For any $\gamma<\omega_{1}$, a set $A \subseteq \omega_{2}$ is $\mathrm{Ha}_{\gamma}$-positive iff there are monotone clopen sets $C_{\alpha n}$ for $\alpha<\gamma, n \in \omega$ such that, letting $E_{\alpha}$ be $\bigcap_{n \in \omega} C_{\alpha n}$ if $\alpha$ has the same parity as $\gamma$ and $-\bigcup_{n \in \omega} C_{\alpha n}$ otherwise, a point $x$ is in $A$ iff the least $\alpha<\gamma$ such that $x \notin E_{\alpha}$ exists and has opposite parity from $\gamma$.

This alternating-series definition, like the other one, has an equivalent form in terms of residues (and the proof of the equivalence is the same). For any monotone $A \subseteq{ }^{\omega_{2}}$, define $R_{\alpha}^{+}(A)$ for $\alpha<\omega_{1}$ as follows: $R_{0}^{+}(A)={ }^{\omega} 2 ; R_{\alpha+1}^{+}(A)=$ $\overline{\left(R_{\alpha}^{+}(A) \cap A\right)} \leq \overline{\cap\left(R_{\alpha}^{+}(A)-A\right)} \leq ;$ and $R_{\lambda}^{+}(A)=\bigcap_{\alpha<\lambda} R_{\alpha}^{+}(A)$ for limit $\lambda$. Then $A$ is

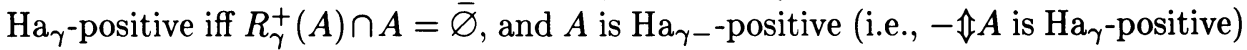
iff $R_{\gamma}^{+}(A) \subseteq A$. (Note that, since the closure of a monotone set is monotone, $R_{1}^{+}(A)=\operatorname{Fr}(A)$ for any monotone $A$. Also note that each set $R_{\alpha}^{+}(A)$ is the difference of two monotone sets; i.e., if $x$ and $z$ are in it and $x<y<z$, then $y$ is in it.)

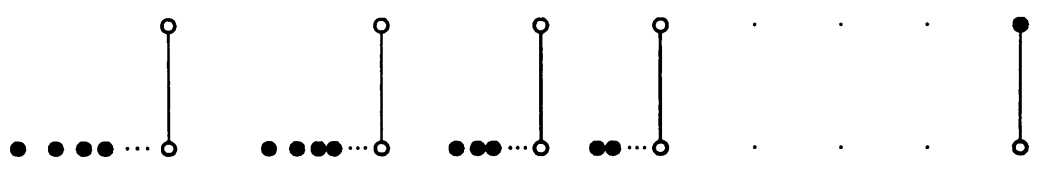

FIGURE 3.8. A monotone nonpositive set in $\mathrm{Ha}_{3}$

Now we can ask the question: is every monotone set in $\mathrm{Ha}_{\gamma} \mathrm{Ha}_{\gamma}$-positive? We already know that the answer is yes for $\gamma \leq 2$. For $\gamma=3$, consider the set $A$ represented by Figure 3.8. Clearly $R_{3}(\alpha)=\varnothing$, so $A$ is in $\mathrm{Ha}_{3} \cap \mathrm{Ha}_{3-}$. But $R_{1}^{+}(A)$ consists of all points shown in the figure, $R_{2}^{+}(A)$ consists of the top row of points and those points on the bottom row which lie below points on the top row, and $R_{3}^{+}(A)$ contains the rightmost point on the top row; therefore, $R_{3}^{+}(A) \cap A \neq \varnothing$, so $A$ is a set in $\mathrm{Ha}_{3} \cap \mathrm{Ha}_{3}$ - which is not $\mathrm{Ha}_{3}$-positive. This answers our question negatively for $\gamma=3$; we will now use a more complicated example to show that the answer is no for all $\gamma>2$.

Suppose $G$ is a finite directed graph with no double edges, cycles of length less than 3 , or isolated vertices. In other words, $G=(V, E)$ where $V$ (the set of vertices) is finite, $E$ (the set of edges) is a subset of $V \times V,(v, u) \in E$ implies $(u, v) \notin E$, and for every $v \in V$ there is $u \in V$ such that either $(v, u) \in E$ or $(u, v) \in E$. Choose an infinite set $W_{v} \subset \omega$ for each $v \in V$ such that $W_{v} \cap W_{u}=\varnothing$ whenever $v \neq u$. For 
each $v \in V$, let $a_{v} \in{ }^{\omega_{2}}$ be the characteristic function of $W_{v}$; for each $e=(v, u) \in E$, let $b_{e}$ be the characteristic function of $W_{v} \cup W_{u}$. Now define a monotone set $A_{G}$ by applying Proposition 2.1 to the sets

$$
\begin{aligned}
& S=\left\{x: \exists(v, u) \in E \quad x \leq b_{(v, u)} \wedge\left(x \geq a_{v} \vee x \geq a_{u}\right)\right\}, \\
& T=\left\{x: \exists(v, u) \in E \quad a_{u}<x \leq b_{(v, u)}\right\} .
\end{aligned}
$$

If $x \in \operatorname{Fr}\left(A_{G}\right)$ is not one of the points $a_{v}$ or $b_{e}$, then there are $v \in V$ and $e \in E$ such that $a_{v}<x<b_{e}$. Hence, there is $n \in \omega$ such that $a_{v}\left\lceil n<x\left\lceil n<b_{e}\lceil n\right.\right.$. Then any $y \in \operatorname{Fr}\left(A_{G}\right)$ such that $y\left\lceil n=x\left\lceil n\right.\right.$ must satisfy $a_{v}<y<b_{e}$, and $y \in A_{G}$ iff $x \in A_{G}$. Therefore, any point in $R_{2}\left(A_{G}\right)$ must be one of the finitely many points $a_{v}$ or $b_{e}$, so $R_{3}\left(A_{G}\right)=\varnothing$ and $A_{G} \in \mathrm{Ha}_{3} \cap \mathrm{Ha}_{3-}$.

Now suppose further that $G$ has no initial vertices (i.e., for every $v \in V$ there is $u \in V$ such that $(u, v) \in E)$; for example, $G$ could be an oriented triangle $(V=\{0,1,2\}, E=\{(0,1),(1,2),(2,0)\})$. If $\alpha$ is such that all of the points $a_{v}$ and $b_{e}$ are in $R_{\alpha}^{+}\left(A_{G}\right)$, then, since every $x \in \operatorname{Fr}\left(A_{G}\right)$ satisfies $a_{v} \leq x \leq b_{e}$ for some $v \in V$ and $e \in E, \operatorname{Fr}\left(A_{G}\right) \subseteq R_{\alpha}^{+}\left(A_{G}\right)$. Now, every point $b_{e}$ is in $A_{G}$ but is the limit of points in $\operatorname{Fr}\left(A_{G}\right)-A_{G}$ (the characteristic functions of $W_{v} \cup\left(W_{u} \cap n\right)$ for $n \in \omega$, where $e=(v, u))$, and every point $a_{v}$ is in $-A_{G}$ but is the limit of points in $\operatorname{Fr}\left(A_{G}\right) \cap A_{G}$ (the characteristic functions of $W_{v} \cup\left(W_{u}-n\right)$ for $n \in \omega$, for some $u$ such that $(u, v) \in E)$; hence, the points $a_{v}$ and $b_{e}$ are in $R_{\alpha+1}^{+}\left(A_{G}\right)$. This proves that the points $a_{v}$ and $b_{e}$ are in $R_{\alpha}^{+}\left(A_{G}\right)$ for all $\alpha$, so:

PROPOSITION 3.20. There are monotone sets in $\mathrm{Ha}_{3} \cap \mathrm{Ha}_{3}$ - which are not $\mathrm{Ha}_{\alpha}$-positive for any $\alpha$.

Thus, the 'most natural' monotone sets in the Wadge classes $\mathrm{Ha}_{\alpha}$ and $\mathrm{Ha}_{\alpha-}$ are not $\leq_{\mathrm{m}}$-maximal for $\alpha>2$; similarly, if we define the 'natural' set in $\mathrm{Ha}_{\alpha+1} \cap$ $\mathrm{Ha}_{\alpha+1}$ - to be the jn ${ }^{+}$of the natural $\mathrm{Ha}_{\alpha}$ set and the natural $\mathrm{Ha}_{\alpha-}$ - set, then the natural set here also fails to be $\leq_{\mathrm{m}}$-maximal if $\alpha>1$. This leads (naturally!?) to the question of whether these classes have $\leq_{m}$-maximal monotone sets at all. This question is still open for the classes $\mathrm{Ha}_{\alpha}$ and $\mathrm{Ha}_{\alpha-}$, but it is settled for most of the classes $\mathrm{Ha}_{\alpha+1} \cap \mathrm{Ha}_{\alpha+1}$ - by the following proposition.

Proposition 3.21. The Wadge class $\mathrm{Ha}_{\alpha+2} \cap \mathrm{Ha}_{\alpha+2}$ - has no $\leq_{\mathrm{m}}$-maximal monotone set, for any countable $\alpha>0$.

Proof. We first do the case $\alpha=1$, and then show how to generalize to higher $\alpha$. Suppose $B$ is a monotone set in $\mathrm{Ha}_{3} \cap \mathrm{Ha}_{3-}$; we will construct a monotone set $A$ in $\mathrm{Ha}_{3} \cap \mathrm{Ha}_{3}$ such that $A \mathbb{Z}_{\mathrm{m}} B$. The sets $R_{2}(B) \cap B$ and $R_{2}(B)-B$ are disjoint and closed, so there is an $n$ such that, for any sequence $s$ of length $n, s^{\omega} 2$ does not meet both of them. Let $G$ be the directed graph with $V=\left\{i \in \omega: i \leq 2^{n}\right\}$ and $E=\left\{(i, j): i<j \leq 2^{n}\right\}$, and let $A=A_{G}$.

Suppose we have a function $f$ witnessing $A \leq_{\mathrm{m}} B$. Since $G$ has more than $2^{n}$ vertices, there must be $i, j \in V$ such that $i<j$ and $f\left(a_{i}\right)\left\lceil n=f\left(a_{j}\right)\lceil n\right.$. Let $x_{k}=f\left(\chi_{W_{i} \cup\left(W_{j} \cap k\right)}\right), y_{k}=f\left(\chi_{W_{j} \cup\left(W_{i}-k\right)}\right)$, and $z=f\left(a_{j}\right)$, where $\chi_{S}$ is the characteristic function of $S$; then $x_{k}, y_{k}$, and $z$ are all in $\operatorname{Fr}(B), x_{k} \notin B, y_{k} \in B$, $z \notin B, x_{k} \leq x_{k+1}, y_{k} \geq y_{k+1}$, and $x_{k} \rightarrow y_{0}$ and $y_{k} \rightarrow z$ as $k \rightarrow \infty$. Hence, $z \in R_{2}(B)-B$. Now let $x_{k}^{\prime}=x_{k} \wedge(z \mid n) \overline{1}$ and $y_{k}^{\prime}=y_{k} \wedge(z \mid n) \overline{1}$, where $x \wedge y$ is the greatest lower bound of $x$ and $y$. Then $x_{k}^{\prime} \leq x_{k+1}^{\prime}, y_{k}^{\prime} \geq y_{k+1}^{\prime}$, and $x_{k}^{\prime} \rightarrow y_{0}^{\prime}$ 
and $y_{k}^{\prime} \rightarrow z$ as $k \rightarrow \infty$. Since $x_{0}=x_{0}^{\prime} \leq x_{k}^{\prime} \leq x_{k}, x_{k}^{\prime}$ must be in $\operatorname{Fr}(B)-B$. Since $y_{k} \rightarrow z$ as $k \rightarrow \infty, y_{k}\lceil n=z\lceil n$ for sufficiently large $k$; for such $k$ we have $y_{0}^{\prime} \geq y_{k}^{\prime}=y_{k}$, so $y_{0}^{\prime} \in B$. Hence, $y_{0}^{\prime} \in R_{2}(B) \cap B$ and $y_{0}^{\prime}\lceil n=z\lceil n$; but this contradicts the choice of $n$. Therefore, $A \mathbb{Z}_{\mathrm{m}} B$, as desired.

We now turn to the case $\alpha>1$. Let $X$ be a set in $\mathrm{Ha}_{\alpha}$ such that there is a point $p$ such that $R_{\alpha}(X)=R_{\alpha}(X \cup\{p\})=\{p\}$. (For example, let $\left\langle\beta_{n}: n \in \omega\right\rangle$ be a nondecreasing sequence cofinal in $\alpha$, choose a set $X_{n}$ for each $n$ such that $X_{n} \in$ $\mathrm{Ha}_{\beta_{n}+1} \cap \mathrm{Ha}_{\beta_{n}+1}$ - but $X_{n} \notin \mathrm{Ha}_{\beta_{n}} \cup \mathrm{Ha}_{\beta_{n}-}$, and let $X=\mathrm{jn}_{\omega}\left(\left\langle X_{n}: n \in \omega\right\rangle\right)$.) Then $X_{\mathrm{b}}$ has the same properties; let $p_{0}$ be the unique point in $R_{\alpha}\left(X_{\mathrm{b}}\right)$ (i.e., $p_{0}=f_{\mathrm{b}}(p)$ ). Suppose $B$ is in $\mathrm{Ha}_{\alpha+2} \cap \mathrm{Ha}_{\alpha+2}$. Choose $n \in \omega$ such that for no sequence $s$ of length $n$ does $s^{\omega} 2$ meet both $R_{\alpha+1}(B) \cap B$ and $R_{\alpha+1}(B)-B$, and define the graph $G$ and the set $A_{G}$ as before. Define $A$ by applying Proposition 2.1 in ${ }^{\omega_{2}} \times{ }^{\omega_{2}}$ with $S=\operatorname{Fr}\left(A_{G}\right) \times \operatorname{Fr}\left(X_{b}\right)$ and $T=\left\{(x, y) \in S: y \in X_{b}\right.$ or $\left(y=p_{0}\right.$ and $\left.\left.x \in A_{G}\right)\right\}$. It is easy to show by induction that $R_{\beta}(A)=\operatorname{Fr}\left(A_{G}\right) \times R_{\beta}\left(X_{\mathrm{b}}\right)$ for all $\beta$ such that $1 \leq \beta \leq \alpha$; in particular, $R_{\alpha}(A)=\operatorname{Fr}\left(A_{G}\right) \times\left\{p_{0}\right\}$, so $R_{\alpha+1}(A)$ is finite and $A \in \mathrm{Ha}_{\alpha+2} \cap \mathrm{Ha}_{\alpha+2}$.

We now complete the proof by showing that $A \underline{\mathrm{m}}_{\mathrm{m}} B$. Suppose $f:{ }^{\omega_{2}} \times{ }^{\omega_{2}} \rightarrow{ }^{\omega_{2}}$ witnesses $A \leq_{\mathrm{m}} B$. As before, there are $i, j \in V$ such that $i<j$ and $f\left(a_{i}, p_{0}\right)\lceil n=$ $f\left(a_{j}, p_{0}\right)\left\lceil n\right.$. Define the points $x_{k}, y_{k}, z, x_{x}^{\prime}$, and $y_{k}^{\prime}$ as before (with $p_{0}$ as an extra argument to $f$; i.e., $z=f\left(a_{j}, p_{0}\right)$, etc.). We will have a contradiction as before once we show that $y_{0}^{\prime} \in R_{\alpha+1}(B) \cap B$ and $z \in R_{\alpha+1}(B)-B$. It is easy to show by induction that $f$ " $R_{\beta}(A) \subseteq R_{\beta}(B)$ for all $\beta$. (Successor step: If $f$ " $R_{\beta}(A) \subseteq$ $R_{\beta}(B)$, then $f$ " $\left(R_{\beta}(A) \cap A\right) \subseteq R_{\beta}(B) \cap B$ and $f$ " $\left(R_{\beta}(A)-A\right) \subseteq R_{\beta}(B)-B$, so $f$ " $\overline{R_{\beta}(A) \cap A} \subseteq \overline{R_{\beta}(B) \cap B}$ and $f$ " $\overline{R_{\beta}(A)-A} \subseteq \overline{R_{\beta}(B)-B}$ by continuity of $f$, so $f^{\text {" }} R_{\beta+1}(A) \subseteq R_{\beta+1}(B)$.) Hence, $z \in R_{\alpha+1}(B)-B$. As before, $y_{0}^{\prime} \in B$ since $y_{0}^{\prime} \geq y_{k}$ for sufficiently large $k$. Also, $x_{k}^{\prime} \notin B$, so it remains to show that $x_{k}^{\prime} \in R_{\alpha}(B)$. Let $\tilde{x}_{k}=\chi_{W_{i} \cup\left(W_{j} \cap k\right)}$. We have $f\left(\tilde{x}_{k}, q\right) \geq f\left(\tilde{x}_{k}, q\right) \wedge\left(f\left(a_{i}, q\right) \mid n\right) \overline{1} \geq f\left(a_{i}, q\right)$ for all $q \in \operatorname{Fr}\left(X_{b}\right)$; since $f\left(\tilde{x}_{k}, q\right)$ and $f\left(a_{i}, q\right)$ are in $\operatorname{Fr}(B), f\left(\tilde{x}_{k}, q\right) \wedge\left(f\left(a_{i}, q\right)\lceil n) \overline{1} \in\right.$ $\operatorname{Fr}(B)$. Also, if $q \neq p_{0}$, then $f\left(\tilde{x}_{k}, q\right) \in B$ iff $f\left(a_{i}, q\right) \in B$ iff $q \in X_{b}$; since $B$ is monotone, $f\left(\tilde{x}_{k}, q\right) \wedge\left(f\left(a_{i}, q\right) \mid n\right) \overline{1} \in B$ iff $q \in X_{b}$. It is now easy to show by induction on $\beta$ that $q \in R_{\beta}\left(X_{b}\right)-\left\{p_{0}\right\}$ implies $f\left(\tilde{x}_{k}, q\right) \wedge\left(f\left(a_{i}, q\right) \mid n\right) \overline{1} \in R_{\beta}(B)$ for $1 \leq \beta<\alpha$. The choice of $X$ guaranteed that, for each $\beta<\alpha, p_{0}$ is a limit of points in $\left(R_{\beta}\left(X_{\mathrm{b}}\right) \cap X_{\mathrm{b}}\right)-\left\{p_{0}\right\}$ and a limit of points in $\left(R_{\beta}\left(X_{\mathrm{b}}\right)-X_{\mathrm{b}}\right)-\left\{p_{0}\right\}$; hence, $x_{k}^{\prime}$ is a limit of points in $R_{\beta}(B) \cap B$ and a limit of points in $R_{\beta}(B)-B$, so $x_{k}^{\prime} \in R_{\beta+1}(B)$. Since this is true for all $\beta<\alpha, x_{k}^{\prime} \in R_{\alpha}(B)$, as desired.

For limit $\lambda$, the question of whether $\mathrm{Ha}_{\lambda+1} \cap \mathrm{Ha}_{\lambda+1}$ - has a $\leq_{\mathrm{m}}$-maximal monotone set remains open.

Proposition 3.25 in $\S I I I .6$ implies that for every $\alpha<\omega_{1}$ there is a $\Delta_{2}^{0}$ set to which all monotone $\mathrm{Ha}_{\alpha}$ sets are monotone reducible. A careful examination of the set produced by the proof for the case of $\mathrm{Ha}_{3}$ (with some of the details streamlined for this special case) shows it to be strictly $\mathrm{Ha}_{\omega+1}$. Of course, this does not rule out the possibility of a simpler set to which all monotone $\mathrm{Ha}_{3}$ sets are monotone reducible.

We now turn to the polarized separation and reduction properties. In the absence of (1.1), one would expect the proofs of these properties to be difficult. Surprisingly, this is not the case for the polarized first separation property.

Proposition 3.22. For any $\alpha<\omega_{1}, \mathrm{PSep}_{\mathrm{I}}\left(\mathrm{Ha}_{\alpha-}\right)$ holds. 
Proof. Suppose $A$ and $Z$ are disjoint sets, both in $\mathrm{Ha}_{\alpha-}$, with $A$ monotone and $Z$ hereditary. Then $R_{\alpha}(A) \subseteq A$, so $R_{\alpha}(A) \leq \subseteq A$; similarly, $R_{\alpha}(Z)_{\leq} \subseteq Z$. Hence, $R_{\alpha}(A) \leq$ and $R_{\alpha}(Z)_{\leq}$are disjoint, so we can apply $\operatorname{PSep}_{\mathrm{I}}(\mathcal{F})$ to get a monotone clopen set $C$ such that $R_{\alpha}(A) \leq \subseteq C \subseteq-R_{\alpha}(Z)_{\leq}$. Let $B=(C-Z) \cup A$. Clearly $B$ is monotone and $A \subseteq B \subseteq-Z$. An easy induction on $\beta$ shows that $R_{\beta}(B)=$ $\left(R_{\beta}(Z) \cap C\right) \cup\left(R_{\beta}(A)-C\right)$ for all $\beta$, so $R_{\alpha}(B)=\varnothing$ and $B \in \mathrm{Ha}_{\alpha} \cap \mathrm{Ha}_{\alpha-}$, as desired.

The reduction property, however, is another story. For one thing, the proof of $\operatorname{PRed}\left(\mathrm{Ha}_{\alpha}\right)$ appears to be somewhat delicate even if one assumes (1.1). To put it more accurately, the proof of the following proposition requires some care.

PROPOSITION 3.23. For any $\gamma<\omega_{1}, \operatorname{Red}^{-1}$ holds for the collection of $\mathrm{Ha}_{\gamma}$ positive sets.

PROOF. We first note that the reason (1.1) is so useful in proofs of $\operatorname{Red}^{-1}$ is that it reduces(!) the problem of reducing all relevant pairs of sets to that of reducing a single such pair. If we are trying to prove $\operatorname{Red}^{-1}$ for the collection $\left\{Y: Y \leq_{\mathrm{m}} X\right\}$, then it suffices to reduce the pair of sets $X \times{ }^{\omega} 2$ and ${ }^{\omega} 2 \times \Uparrow X$ in the space ${ }^{\omega_{2}} \times{ }^{\omega_{2}} 2$. For suppose we have any two sets $Y, \tilde{Y}$ such that $Y \leq_{\mathrm{m}} X$ and $\hat{Y} \tilde{Y} \leq_{\mathrm{m}} X$; let $f$ and $g$ be functions witnessing these reducibilities. Let $h(x)=(f(x), \hat{\mathbb{1}}(g(\hat{\Downarrow}(x))))$; then $h$ simultaneously witnesses $Y \leq_{\mathrm{m}} X \times{ }^{\omega_{2}}$ and $\tilde{Y} \leq_{\mathrm{m}}{ }^{\omega} 2 \times \hat{\mathbb{N}} X$, so from a reducing pair for $X \times{ }^{\omega_{2}}$ and ${ }^{\omega_{2}} \times \hat{\mathbb{N}} X$ we can obtain a reducing pair for $Y$ and $\tilde{Y}$ by taking inverse images under $h$.

The collection of $\mathrm{Ha}_{\gamma}$-positive sets has the form $\left\{B: B \leq_{\mathrm{m}} C\right\}$, where the monotone set $C$ can be defined as follows: let $W_{\alpha}$ for $\alpha<\gamma$ be disjoint infinite subsets of $\omega$, and let $C$ be the set of all $x \in{ }^{\omega} 2$ such that there is an $\alpha<\gamma$ of opposite parity from $\gamma$ such that $x(n)=1$ for some $n \in W_{\alpha}$ and there is no $\beta<\alpha$ of the same parity as $\gamma$ such that $x(n)=0$ for some $n \in W_{\beta}$. Let $A=C \times{ }^{\omega}{ }^{\omega}$ and $Z={ }^{\omega} 2 \times \hat{\mathbb{1}} C$; it will suffice to reduce the sets $A, Z \subseteq{ }^{\omega_{2}} \times{ }^{\omega_{2}}$.

Define functions $h_{0}, h_{1}$ : ${ }^{\omega} 2 \rightarrow \gamma+1$ by: if there is a $\beta<\gamma$ of the same parity as $\gamma$ such that $x(n)=i$ for some $n \in W_{\beta}$, let $h_{i}(x)$ be the least such $\beta$; otherwise, let $h_{i}(x)=\gamma$. We now define sets $A^{\prime}, Z^{\prime}$ to reduce $A$ and $Z$. Any $(x, y) \in A-Z$ is put in $A^{\prime}$ and any $(x, y) \in Z-A$ in $Z^{\prime}$, of course. If $(x, y) \in A \cap Z$, let $n_{0}(x)$ be the least $n$ such that $x(n)=1$ and $n \in W_{\beta}$ for some $\beta<h_{0}(x)$ of opposite parity from $\gamma$ (such an $n$ exists since $x \in C$ ), and let $n_{1}(y)$ be the least $n$ such that $y(n)=0$ and $n \in W_{\beta}$ for some $\beta<h_{1}(y)$ of opposite parity from $\gamma$; put $(x, y)$ in $A^{\prime}$ if $n_{0}(x)<n_{1}(y), Z^{\prime}$ otherwise.

If $(x, y) \leq(\hat{x}, \hat{y})$ are points in $A \cap Z$, then it is easy to see that $h_{0}(x) \leq h_{0}(\hat{x})$, so $n_{0}(x) \geq n_{0}(\hat{x})$; similarly, $n_{1}(y) \leq n_{1}(\hat{y})$. It follows that $A^{\prime}$ is monotone and $Z^{\prime}$ is hereditary.

We now prove by induction on $\alpha$ that: if $(x, y) \in R_{\alpha}^{+}\left(A^{\prime}\right) \cap A^{\prime}$ and $n_{0}(x) \in W_{\beta}$, then $\beta \geq \alpha$; if $(x, y) \in R_{\alpha}^{+}\left(A^{\prime}\right)-A^{\prime}$, then $h_{0}(x) \geq \alpha$. These are both obvious for $\alpha=0$. If $\alpha>0$, let $\left\langle\alpha_{k}: k \in \omega\right\rangle$ be a nondecreasing sequence such that $\sup _{k \in \omega}\left(\alpha_{k}+1\right)=\alpha$. If $(x, y) \in R_{\alpha}^{+}\left(A^{\prime}\right) \cap A^{\prime}$, then there are $(\tilde{x}, \tilde{y}) \geq(x, y)$ and $\left(x_{k}, y_{k}\right) \in R_{\alpha_{k}}^{+}\left(A^{\prime}\right)-A^{\prime}$ such that $\left(x_{k}, y_{k}\right) \rightarrow(\tilde{x}, \tilde{y})$ as $k \rightarrow \infty$. Let $n=n_{0}(x)$, and let $\beta$ be such that $n \in W_{\beta}$. Since $(x, y) \in A^{\prime}, n_{1}(y)$ must be greater than $n$ if it exists, so, for each $i \leq n$ such that $y(i)=0$ and $i \in W_{\beta^{\prime}}$ for some $\beta^{\prime}$ of opposite parity from $\gamma$, there is $j \in \omega$ such that $y(j)=1$ and $j \in W_{\beta^{\prime \prime}}$ for some $\beta^{\prime \prime}<\beta$ 
of the same parity as $\gamma$. Fix $M>n$ such that for each such $i \leq n$ there is such a $j$ which is less than $M$. For all sufficiently large $k$ we have $x_{k}\lceil M=\tilde{x}\lceil M$ and $y_{k}\left\lceil M=\tilde{y}\left\lceil M\right.\right.$; since $x_{k}\left\lceil M=\tilde{x}\left\lceil M \geq x\left\lceil M\right.\right.\right.$ and $\left(x_{k}, y_{k}\right) \notin A^{\prime}, \beta$ must be greater than $h_{0}\left(x_{k}\right)$, which is at least $\alpha_{k}$ by the induction hypothesis. Therefore, $\beta \geq \alpha_{k}+1$ for all sufficiently large $k$, so $\beta \geq \alpha$. On the other hand, if $(x, y) \in R_{\alpha}^{+}\left(A^{\prime}\right)-A^{\prime}$, then there are $(\tilde{x}, \tilde{y}) \leq(x, y)$ and $\left(x_{k}, y_{k}\right) \in R_{\alpha_{k}}^{+}\left(A^{\prime}\right) \cap A^{\prime}$ such that $\left(x_{k}, y_{k}\right) \rightarrow(\tilde{x}, \tilde{y})$ as $k \rightarrow \infty$. Then $n_{0}\left(x_{k}\right) \in W_{\beta}$ for some $\beta \geq \alpha_{k}$, so since $\left(x_{k}, y_{k}\right) \in A$ we have $h_{0}\left(x_{k}\right)>\alpha_{k}$. Since $\alpha_{k} \leq \alpha_{j}$ for $j>k$ and the set of $z$ such that $h_{0}(z)>\alpha_{k}$ is closed, $h_{0}(\tilde{x})>\alpha_{k}$, so $h_{0}(x)>\alpha_{k}$; since this is true for all $k, h_{0}(x) \geq \alpha$. This completes the induction.

In particular, no point $(x, y)$ can be in $R_{\gamma}^{+}\left(A^{\prime}\right) \cap A^{\prime}$, so $A^{\prime}$ is $\mathrm{Ha}_{\gamma}$-positive; the proof that $\mathbb{\Downarrow} Z^{\prime}$ is $\mathrm{Ha}_{\boldsymbol{\gamma}}$-positive is similar, so we are done.

Of course, this says nothing about general monotone $\mathrm{Ha}_{\gamma}$ sets. It is unknown whether $\operatorname{PRed}\left(\mathrm{Ha}_{\gamma}\right)$ holds for all $\gamma<\omega_{1}$; however, we can prove:

Proposition 3.24. For $\gamma \leq 3$, PPwo $\left(\mathrm{Ha}_{\gamma}\right.$ ) (and hence PRed $\left(\mathrm{Ha}_{\gamma}\right)$ ) holds.

PROOF. The case $\gamma=0$ is trivial, and we have already done the case $\gamma=1$. For $\gamma=2$, we saw in subsection III.2.1 that any monotone $\mathcal{F}_{\rho}$ set can be expressed in the form $A=A_{0} \cap A_{1}$ with $A_{0}$ closed, $A_{1}$ open, and both monotone; if $\preceq$ is the prewellordering of $A_{1}$ obtained from $\operatorname{PPwo}(\mathcal{G})$, then $\preceq\lceil A$ is a suitable prewellordering of $A$, since

$x \in A \wedge(y \notin A \vee x \preceq y)$

$$
\Leftrightarrow x \in A_{0} \wedge\left(x \in A_{1} \wedge\left(y \notin A_{0} \vee\left[x \in A_{1} \wedge\left(y \notin A_{1} \vee x \preceq y\right)\right]\right)\right)
$$

and similarly for $y \npreceq x$. Now suppose $A \in \mathrm{Ha}_{3}$. The sets in $\mathrm{Ha}_{3}$ are those of the form $\left(X_{0} \cup X_{1}\right) \cap X_{2}$, where $X_{0}$ and $X_{2}$ are open and $X_{1}$ is closed; in fact, for $A$, we have $A=\left(A_{0} \cup X_{1}\right) \cap A_{2}$, where $A_{0}=-\overline{-A}$ (the interior of $\left.A\right), X_{1}=\overline{\operatorname{Fr}(A) \cap A}$, and $A_{2}=-\left(R_{2}(A)-A\right)_{\leq}$(By Proposition 1.2(a), $A_{0}$ is monotone and $A_{2}$ is open.) Let $\preceq_{0}$ and $\preceq_{2}$ be obtained by applying $\operatorname{PPwo}(\mathcal{G})$ to $A_{0}$ and $A_{2}$, respectively. Now define $\preceq$ on $A$ by: for $x, y \in A$,

$$
x \preceq y \Leftrightarrow x \preceq_{0} y \vee\left(y \notin A_{0} \wedge\left(x \in A_{0} \vee x \preceq_{2} y\right)\right) .
$$

It is easy to see that $\preceq$ is a prewellordering, and $x \in A$ and $x \leq y$ imply $y \preceq x$. Finally, if $\psi(x, y)$ is one of the formulas $x \in A \wedge(y \notin A \vee x \preceq y)$ and $x \in A \wedge(y \notin$ $A \vee y \npreceq x)$, then $\{(x, y): \psi(x, y)\}$ is $\mathrm{Ha}_{3}$, since

$$
\psi \Leftrightarrow\left(\psi_{0} \vee\left(x \in X_{1} \wedge y \notin A_{0}\right)\right) \wedge\left(x \in A_{2} \wedge\left(\psi_{0} \vee \psi_{2} \vee y \notin X_{1}\right)\right),
$$

where $\psi_{i}$ is $\psi$ with $A$ and $\preceq$ replaced by $A_{i}$ and $\preceq_{i}$. (Use the fact that $A_{0} \subseteq A_{2}$.)

5. The rest of the Borel hierarchy. This section is rather short, because most of the interesting questions at this level are still open.

The Borel and Hausdorff hierarchies are analogous in many ways, so looking at the Hausdorff hierarchy can give hints as to the situation for the Borel hierarchy. For example, up to level 2 in both hierarchies the natural monotone set is $\leq_{m^{-}}$maximal, and the polarized separation and reduction properties hold where the ordinary properties hold. According to this analogy, these nice properties should 
break down at level 3 in the Borel hierarchy; in fact, we would expect to find a monotone $\Delta_{3}^{0}$ set which is not $\boldsymbol{\Sigma}_{\alpha}^{0}$-positive for any $\alpha<\omega_{1}$.

The analogy, however, is not that strong; it turns out that every monotone Borel set is $\Sigma_{\alpha}^{0}$-positive for some $\alpha$. One way to prove this is to make an easy modification in the usual proof of $\operatorname{Sep}_{\mathrm{I}}\left(\Sigma_{1}^{1}\right)[\mathbf{1 2}, 2 \mathrm{E}$.1]; this gives the slightly stronger result that, if the analytic sets $X$ and $Y$ can be separated by a monotone set (i.e., $X \leq \cap Y=\varnothing$ ), then they can be separated by a $\Sigma_{\alpha}^{0}$-positive set for some $\alpha$. (A. Louveau has noted that a variant of this argument can be based on the Choquet capacitability theorem.) Another way, as S. Dyck and others have noted, is to deduce it from an analogue of Lyndon's interpolation theorem for an infinitary propositional logic; in particular, it follows easily from the Lopez-Escobar interpolation theorem (or, rather, from its corollary, the homomorphism theorem [9, Theorem 5.1]).

We do, however, get weaker negative results starting at level 3. There are monotone $\Delta_{3}^{0}$ sets which are not $\Sigma_{3}^{0}$-positive and, dually, monotone $\Delta_{3}^{0}$ sets which are not $\Pi_{3}^{0}$-positive; applying jn ${ }^{+}$gives a monotone $\Delta_{3}^{0}$ set which is neither $\Sigma_{3}^{0}$-positive nor $\Pi_{3}^{0}$-positive. Also, for each finite $n \geq 3$, there are monotone $\Sigma_{n}^{0}$ sets which are not $\boldsymbol{\Pi}_{n+1}^{0}$-positive, and monotone $\boldsymbol{\Pi}_{n}^{0}$ sets which are not $\boldsymbol{\Sigma}_{n+1}^{0}$-positive. These results are all proved by the same method; since the general theory in this paper was not needed, the results have been published separately [2].

This is all that is known (by me, at least) at the moment. Among the interesting questions left open are: Is every monotone $\Sigma_{\omega}^{0}$ set $\Sigma_{\omega}^{0}$-positive? Is there a maximal $\Sigma_{3}^{0}$ monotone degree? Is there, for every $\alpha<\omega_{1}$, a monotone $\Delta_{3}^{0}$ set which is not $\Sigma_{\alpha}^{0}$-positive?

It is also unknown whether $\operatorname{PSep}_{\mathrm{I}}\left(\boldsymbol{\Pi}_{\alpha}^{0}\right)$ and $\operatorname{PRed}\left(\boldsymbol{\Sigma}_{\alpha}^{0}\right)$ hold for $\alpha \geq 3$. The usual proof of $\operatorname{Red}\left(\Sigma_{\alpha}^{0}\right)$ is easily converted to a proof of $\operatorname{Red}^{-\uparrow}$ for the $\Sigma_{\alpha}^{0}$-positive sets, but this is not much help. It would suffice if we knew that every monotone $\Sigma_{\alpha}^{0}$ set was a countable union of monotone sets each of which was $\Pi_{\beta}^{0}$ for some $\beta<\alpha$, but this is false for $\alpha=3$ (since it would imply that every monotone $\Sigma_{3}^{0}$ set is $\Sigma_{3}^{0}$-positive) and open for $\alpha>3$. (By the way, the simple method of taking upward closures of $\Pi_{\beta}^{0}$ sets which are not necessarily monotone will not work. There is a $\Pi_{2}^{0}$ set $X \subseteq{ }^{\omega_{2}} \times{ }^{\omega_{2}}$ such that $Y=\{y: \exists x(x, y) \in X\}$ is not Borel; if $f_{\mathrm{b}}$ is as in section II.4 and $\psi:{ }^{\omega_{2}} \times{ }^{\omega_{2}} \rightarrow{ }^{\omega_{2}} 2$ is the canonical isomorphism, then $X^{\prime}=f_{\mathrm{b}}$ " $\psi$ " $X$ is $\Pi_{2}^{0}$ but $\left(X^{\prime}\right) \leq$ is not Borel, since $y \in Y$ iff $\left(f_{b}(\psi(\overline{0}, y)) \vee \overline{1100}\right) \in\left(X^{\prime}\right) \leq$.)

6. The projective hierarchy. Most of the natural questions about $\leq_{m}$ at the projective level have easy answers, at least as far as the corresponding questions about $\leq_{\mathrm{w}}$ can be answered.

It will be convenient to work in spaces of the form ${ }^{\omega_{2}} \times\left({ }^{\omega} \omega\right)^{k}$ as well as in ${ }^{\omega_{2}}$. Define $\leq$ on such spaces by letting $(x, \vec{y}) \leq\left(x^{\prime}, \vec{y}^{\prime}\right)$ iff $x \leq x^{\prime}$ and $\vec{y}=\vec{y}^{\prime}$. Then define monotone sets, $\leq_{\mathrm{m}}$, monotone and Wadge degrees and classes, etc. as usual.

Recall that a set $X \subseteq{ }^{\omega} 2 \times\left({ }^{\omega} \omega\right)^{k}$ is $\Sigma_{n}^{1}$ iff there is a $\Pi_{n-1}^{1}$ set $Y \subseteq{ }^{\omega} 2 \times\left({ }^{\omega} \omega\right)^{k+1}$ such that $X$ is the projection of $Y$; i.e., $(x, \vec{y}) \in X$ iff there is a $z$ such that $(x, \vec{y}, z) \in$ $Y$. (The $\Pi_{0}^{1}$ sets are just the closed sets. All of the facts and definitions we need about pointclasses and the projective hierarchy can be found in Moschovakis [12].) The key fact (which was pointed out to me by A. Kechris) is that any monotone $\Sigma_{n}^{1}$ set is the projection of a monotone $\Pi_{n-1}^{1}$ set. If $X$ is the projection of $Y$ and 
$X$ is monotone, then

$$
\begin{aligned}
(x, \vec{y}) \in X & \Leftrightarrow \exists x^{\prime}\left(x^{\prime} \leq x \wedge\left(x^{\prime}, \vec{y}\right) \in X\right) \\
& \Leftrightarrow \exists x^{\prime}\left(x^{\prime} \leq x \wedge \exists z\left(x^{\prime}, \vec{y}, z\right) \in Y\right) \\
& \Leftrightarrow \exists z(x, \vec{y}, z) \in Y^{\prime},
\end{aligned}
$$

where (letting $\langle$,$\left.\rangle denote some nice pairing function on { }^{\omega} \omega\right) Y^{\prime}$ is the set of all $\left(x, \vec{y},\left\langle z, x^{\prime}\right\rangle\right)$ such that $x^{\prime} \in{ }^{\omega_{2}}, x^{\prime} \leq x$, and $\left(x^{\prime}, \vec{y}, z\right) \in Y$; it is easy to see that $Y^{\prime}$ is monotone and $\Pi_{n-1}^{1}$. More generally, if a monotone set $X$ is the projection of a set $Y$ in a Wadge class $\Gamma$ which is closed under intersections with closed sets, then $X$ is the projection of a monotone set $Y^{\prime} \in \Gamma$. (Any $\Gamma \supset \Delta_{2}^{0}$ has this property [3, Lemma 1.7].)

We now define $\boldsymbol{\Sigma}_{n}^{1}$-positive and $\boldsymbol{\Pi}_{n}^{1}$-positive sets in a natural way. We will give the case $n=2$ here; the general case is no harder except notationally. If $X \subseteq{ }^{\omega_{2}} \times\left({ }^{\omega} \omega\right)^{k}$ is $\Sigma_{2}^{1}$, then it is the projection of the complement of the projection of a closed set $Y \subseteq{ }^{\omega_{2}} \times\left({ }^{\omega} \omega\right)^{k+2}$. Let $T \subseteq{ }^{<\omega_{2}} \times\left({ }^{<\omega} \omega\right)^{k}$ be a tree such that $Y=[T] ;$ then

$$
\begin{aligned}
(x, \vec{y}) \in X & \Leftrightarrow \exists z_{0} \forall z_{1}\left(x, \vec{y}, z_{0}, z_{1}\right) \notin Y \\
& \Leftrightarrow f \exists z_{0} \forall z_{1} \exists m\left(x \left\lceilm, y_{0}\left\lceil m, y_{1}\left\lceil m, \ldots, y_{k-1}\left\lceil m, z_{0}\right\rceil m, z_{1}\right\rceil m\right) \notin T\right.\right. \\
& \Leftrightarrow \exists z_{0} \forall z_{1} \exists m(x, \vec{y}) \in U_{z_{0}\left\lceil m, z_{1}\lceil m\right.},
\end{aligned}
$$

where the sets $U_{s_{0}, s_{1}}$ are clopen subsets of $\omega_{2} \times\left(\omega_{\omega}\right)^{k}$. This expresses $X$ as the result of applying a certain Boolean operation to clopen sets $U_{s_{0}, s_{1}}$, and it is clear that any set $X$ obtained from clopen sets $U_{s_{0}, s_{1}}$ via this Boolean operation is $\Sigma_{2}^{1}$. The operation is clearly monotone, so we define the collection of $\Sigma_{2}^{1}$-positive sets to be the result of applying this operation to all sequences (indexed by $(<\omega \omega)^{2}$ ) of monotone clopen sets; that is, a set $X$ is $\Sigma_{2}^{1}$-positive iff there are monotone clopen sets $U_{s_{0} s_{1}}$ such that $X=\left\{(x, \vec{y}): \exists z_{0} \forall z_{1} \exists m(x, \vec{y}) \in U_{z_{0}\left\lceil m, z_{1}\lceil m\right.}\right\}$. Similarly, we define a set $X$ to be $\Pi_{2}^{1}$-positive iff there are monotone clopen sets $U_{s_{0} s_{1}}$ such that $X=\left\{(x, \vec{y}): \forall z_{0} \exists z_{1} \forall m(x, \vec{y}) \in U_{z_{0}\left\lceil m, z_{1} \backslash m\right.}\right\}$. For other $n, \boldsymbol{\Sigma}_{n}^{1}$-positive and $\boldsymbol{\Pi}_{n^{-}}^{\mathbf{1}}$ positive are defined analogously. (Note that the Boolean operation used to define $\boldsymbol{\Sigma}_{1}^{1}$-positive is Suslin's operation $A$.)

Note that, in the preceding paragraph, if $X$ is monotone, then the key fact above (along with its dual) allows us to choose $Y$ to be hereditary, so the sets $U_{s_{0} s_{1}}$ will be monotone. Therefore, any monotone $\Sigma_{2}^{1}$ set is $\Sigma_{2}^{1}$-positive; and the same argument applies to $\boldsymbol{\Sigma}_{n}^{1}$ and $\boldsymbol{\Pi}_{n}^{1}$ for all $n$.

The separation and reduction properties at the projective levels are usually obtained as consequences of the prewellordering property, rather than directly. The basic facts about these properties and their polarized versions hold over ${ }^{\omega} 2 \times\left({ }^{\omega} \omega\right)^{k}$ as well as over ${ }^{\omega_{2}}$ (although the proof that $\operatorname{PPwo}(\Gamma)$ implies $\operatorname{PRed}(\Gamma)$ should be

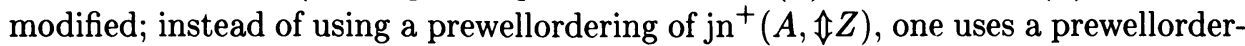
ing of

$$
\{(x, \vec{y}, n z):(n=0 \wedge(x, \vec{y}, z) \in A) \vee(n>0 \wedge(\hat{\Downarrow}(x), \vec{y}, z) \in Z)\} .)
$$

Also, a number of facts about Pwo $(\Gamma)$ can be seen to hold also for $\mathrm{PPwo}(\Gamma)$ simply by using the key fact above and noting that the resulting norms are 'polarized.' Some of the resulting facts about $\mathrm{PPwo}(\Gamma)$ are as follows; the references are to the 
corresponding facts about $\operatorname{Pwo}(\Gamma)$. Suppose $\Gamma$ is an adequate pointclass. If $\Gamma$ is closed under $\forall^{\omega} \omega$, then PPwo $(\Gamma)$ implies PPwo $\left(\exists^{\omega} \omega \Gamma\right)[\mathbf{1 2}, 4 \mathrm{~B} .3]$. If $\Gamma$ is closed under $\exists^{\omega} \omega$, and all games on $\omega$ with payoff set in $\boldsymbol{\Gamma} \cap \boldsymbol{\Gamma}_{-}$are determined, where $\boldsymbol{\Gamma}$ is the collection of sets of the form $\{\vec{y}:(\vec{y}, z) \in X\}$ for $z \in{ }^{\omega} \omega$ and $X \in \Gamma$, then $\operatorname{PPwo}(\Gamma)$ implies PPwo $\left(\forall^{\omega} \omega\right)$ ) $[\mathbf{1 2}, 6 \mathrm{~B} .1]$. (Therefore, $\operatorname{PPwo}\left(\boldsymbol{\Pi}_{1}^{1}\right)$ and $\operatorname{PPwo}\left(\boldsymbol{\Sigma}_{2}^{1}\right)$ hold, and determinacy for all projective sets implies that $\operatorname{PPwo}\left(\boldsymbol{\Sigma}_{2 n}^{1}\right)$ and $\operatorname{PPwo}\left(\boldsymbol{\Pi}_{2 n+1}^{1}\right)$ hold for all $n$.) If $\omega_{\omega} \subseteq L$, then $\operatorname{PPwo}\left(\Sigma_{n}^{1}\right)$ holds for all $n \geq 2[\mathbf{1 2}, 5 \mathrm{~A} .3]$.

This seems to settle our main questions at this level (as far as possible); there is little to say about monotone reducibility other than analogues of facts about Wadge reducibility once closure under $\exists^{\omega} \omega$ or $\forall^{\omega} \omega$ is assumed. One exception is the following curious proposition:

PROPOSITION 3.25. Suppose $\boldsymbol{\Gamma}$ is a Wadge class closed under binary unions and intersections and $\forall^{\omega 2}$ such that $\operatorname{PSep}_{\mathrm{I}}(\boldsymbol{\Gamma})$ holds. Then, for any $X \in \Delta=$ $\Gamma \cap \Gamma_{-}$, there is a monotone set $A \in \Delta$ such that, for any monotone $B \leq_{\mathrm{w}} X$, $B \leq_{\mathrm{m}} A$.

PROOF. The result is trivial if $\Delta$ has a maximal monotone degree, so we may assume $\Gamma \nsubseteq \mathcal{F}_{\rho}$ and hence, by closure under $\forall^{\omega}{ }^{2}, \Pi_{2}^{0} \subseteq \Gamma$. Fix a coding of continuous functions $f:{ }^{\omega} 2 \rightarrow{ }^{\omega} 2$ by elements of ${ }^{\omega_{2}}$ such that the sets

$$
\begin{aligned}
& \{(x, y, z): z \text { codes a function } f \text { and } f(x)=y\} \\
& \{(x, y, z): z \text { codes a function } f \text { and } f(x) \neq y\}
\end{aligned}
$$

are $\Pi_{2}^{0}$. (For example, one could look at $z$ as the characteristic function of a set of pairs $(s, t) \in\left({ }^{<\omega} 2\right)^{2}$, with $z((s, t))=1$ meaning $x \supset s \Rightarrow f(x) \supset t$.)

Suppose $X \in \Delta$, and let

$$
\begin{aligned}
& R=\left\{z \in{ }^{\omega} 2: z \text { codes a function } f\right. \text { and } \\
& \left.\quad \forall x \forall x^{\prime} \forall y \forall y^{\prime}\left(\left(f(x)=y \wedge f\left(x^{\prime}\right)=y^{\prime} \wedge x \leq x^{\prime} \wedge y \in X\right) \Longrightarrow y^{\prime} \in X\right)\right\} .
\end{aligned}
$$

Then $R$ is easily seen to be in $\Gamma$. Define sets $A^{\prime}, Z^{\prime} \subseteq{ }^{\omega} 2$ by

$$
\begin{aligned}
& A^{\prime}=\left(\left(f_{b} " \omega_{2}\right) \leq \times{ }^{\omega} 2\right)-\left\{\left(f_{b}(z), x\right): z \notin R \vee \neg(z \operatorname{codes} f \wedge f(x) \in X)\right\}, \\
& Z^{\prime}=\left(\left(f_{b} " \omega_{2}\right)_{\leq} \times{ }^{\omega} 2\right)-\left\{\left(f_{b}(z), x\right): z \notin R \vee \neg(z \operatorname{codes} f \wedge f(x) \notin X)\right\} .
\end{aligned}
$$

Then $A^{\prime}$ is monotone, $Z^{\prime}$ is hereditary, $A^{\prime}, Z^{\prime} \in \Gamma$, and $A^{\prime} \cap Z^{\prime}=\varnothing$. Apply PSep $_{\mathrm{I}}(\Gamma)$ to get a monotone set $A \in \Delta$ such that $A^{\prime} \subseteq A \subseteq-Z^{\prime}$. To see that this $A$ (or its image under the canonical homeomorphism from ${ }^{\omega_{2}} \times{ }^{\omega_{2}}$ to ${ }^{\omega_{2}}$ ) works, suppose $B \leq_{\mathrm{w}} X$, let $f$ be a witness to this, and let $z$ be a code for $f$. Then $x \in B$ iff $\left(f_{b}(z), x\right) \in A^{\prime}$ iff $\left(f_{b}(z), x\right) \notin Z^{\prime}$, so $x \in B$ iff $\left(f_{b}(z), x\right) \in A$; therefore, $B \leq_{\mathrm{m}} A$, as desired.

Among the pointclasses to which this proposition applies are $\Pi_{2}^{1}$ and, assuming projective determinacy, $\Pi_{2 n}^{1}$ for all $n>1$. Note that the proposition only considers compact spaces (products of ${ }^{\omega_{2}}$ ); hence, it also applies to the pointclass $\boldsymbol{\Pi}_{2}^{0}$. This gives the result, mentioned in §III.4, that for every $\alpha<\omega_{1}$ there is a monotone $\boldsymbol{\Delta}_{2}^{0}$ set to which all monotone $\mathrm{Ha}_{\alpha}$ sets are monotone reducible.

\section{SUMMARY AND OPEN PROBLEMS}

In the course of our study of monotone reducibility, we have noticed a number of similarities to Wadge reducibility and a number of differences. Among the similarities are some analogous general constructions (duality and joins); also, in cases 
when the natural notion of 'positive' is equivalent to 'monotone,' a Wadge class has the same sort of structural properties (the separation and reduction properties) under monotone reducibility that it has under Wadge reducibility. On the other hand, if 'positive' and 'monotone' do not coincide, it is difficult or impossible to prove the monotone versions of these properties. One of the greatest differences between the two reducibilities is that the nice semilinear arrangement of the Borel Wadge degrees breaks down completely for the monotone degrees at a very low level; in fact, the examples obtained at the $\mathcal{F}_{\rho}$ level for monotone degrees resemble those that one can get for Wadge reducibility only by using a well-ordering of ${ }^{\omega_{2}}$.

Among the many problems that remain open, the most interesting are probably those at the Borel level mentioned in §III.5. A few such questions, however, can be asked more generally. For example, is there a Wadge class $\Gamma$ such that $\operatorname{Red}(\Gamma)$ holds but PRed( $\Gamma)$ does not? Is there a nonselfdual Wadge class without a maximal monotone degree? Inversely, is there a Wadge degree for which the natural monotone degree is not maximal, but which does have a maximal monotone degree?

Finally, there are two possible weakenings of our restrictions which may (or may not) be worth exploring. First, one could consider $\leq_{m}$ for subsets of ${ }^{\omega} 2$ which are not necessarily monotone. In this case, the clopen sets will divide up into $\omega$ levels, each having two monotone degrees, and the arrangement of the closed sets will probably be quite complex.

Second, one could look at monotone reducibility on partially ordered topological spaces other than $\left({ }^{\omega} 2, \leq\right)$. We have already seen an example of this in §III.6. It is unlikely that many of the results obtained here will go through for more general spaces; however, one might examine such spaces in order to decide which results depended on which special properties of $\left({ }^{\omega} 2, \leq\right)$. For example, duality and the 'flat' operation clearly use the symmetry of the partial order, the join construction depends on the homogeneity of the space, and quite a few arguments have involved compactness; looking at other spaces would give an idea of which of these properties are essential.

ACKNOWLEDGMENTS. I would like to thank J. W. Addison for posing the problems which led me to this area, and A. S. Kechris for helpful discussions.

\section{REFERENCES}

1. D. Cenzer, Monotone reducibility and the family of infinite sets, J. Symbolic Logic 49 (1984), 774-782.

2. R. Dougherty, Monotone but not positive subsets of the Cantor space, J. Symbolic Logic 52 (1987), 817-818.

3. __ Sequential discreteness and clopen-I-Boolean classes, J. Symbolic Logic 52 (1987), 232242.

4. F. Hausdorff, Set theory, Chelsea, New York, 1957.

5. H. Keisler, Universal homogeneous Boolean algebras, Michigan Math. J. 13 (1966), 129-132.

6. K. Kunen, Set theory, North-Holland, Amsterdam, 1980.

7. R. Laver, Linear orders in $(\omega)^{\omega}$ under eventual dominance, Logic Colloquium '78 (M. Boffa et al., eds.), North-Holland, Amsterdam, 1979.

8. , On Fraïssé's order type conjecture, Ann. of Math. 93 (1971), 89-111.

9. E. Lopez-Escobar, An interpolation theorem for denumerably long formulas, Fund. Math. $\mathbf{5 7}$ (1965), 253-274.

10. A. Louveau and J. Saint Raymond, Borel classes and closed games: Wadge-type and Hurewicztype results, Trans. Amer. Math. Soc. 304 (1987), 431-467. 
11. A. Miller, Some problems in set theory and model theory, Doctoral Dissertation, Univ. of California, Berkeley, 1978.

12. Y. Moschovakis, Descriptive set theory, North-Holland, Amsterdam, 1980.

13. J. Rosenstein, Linear orderings, Academic Press, New York, 1982.

14. W. Wadge, Reducibility and determinateness on the Baire space, Doctoral Dissertation, Univ. of California, Berkeley, 1983.

Department of Mathematics, California institute of Technology, PasaDENA, CALIFORNIA 91125

Current address: Department of Mathematics, University of California, Los Angeles, California 90024 